\title{
Measurement of a new half-lifetime lower limit for neutrinoless double-beta decay in Xe-136 using the full EXO-200 phase 1 data set and improvements to barium tagging for nEXO
}

\author{
by \\ Warren Cree, B.Sc. \\ A thesis submitted to the \\ Faculty of Graduate and Postdoctoral Affairs \\ in partial fulfillment of the requirements for the degree of \\ Master of Science, Particle Physics \\ Ottawa-Carleton Institute for Physics \\ Department of Physics \\ Carleton University \\ Ottawa, Ontario \\ July, 2017 \\ (C) Copyright \\ Warren Cree, 2017
}




\begin{abstract}
Several major experiments are currently underway attempting to observe neutrinoless double-beta decay $(0 \nu \beta \beta)$. This decay is of interest in the physics community because a measurement of it's half-life would lead directly to a measurement of the effective Majorana-neutrino mass and therefore the absolute neutrino mass scale. Furthermore, the decay is interesting because it demonstrates non-conservation of lepton number and the Majorana nature of neutrinos, two features which are not forbidden by the Standard Model but have not been observed experimentally. This work focuses on improvements to two such experiments, EXO-200 and it's successor, nEXO. An improved analysis technique for EXO-200 is developed using Boosted Decision Trees, leading to a $12 \%$ improvement to sensitivity and a $13 \%$ improvement to the $0 \nu \beta \beta$ half-life lower limit. Furthermore, improvements are made to the nEXO barium tagging system producing a 50\% improvement in signal-to-background ratio as well as some improvement to the continuous excitation technique.
\end{abstract}




\section{Acknowledgments}

I would like to thank my supervisors, Dr. Thomas Koffas and Dr. David Sinclair without whom this work would not have been possible. I would also like to thank the EXO-200 analysis group, and in particular Dr. Caio Licciardi whose guidance in learning about EXO-200 and developing this work was invaluable. Furthermore I extend my gratitude to Philippe Gravelle, Yves Baribeau and Rodney Schnarr whose hard work and expertise kept my lab (and many others) running smoothly throughout the years. A special thanks goes to my colleague Ryan Killick who not only showed me the ropes of barium tagging but whose hard work laid the foundation for a large portion of this thesis. Finally, thanks to my friends and family who pushed me to succeed and provided a welcome release from stress over the years. 


\section{Table of Contents}

Abstract $\quad$ ii

Acknowledgments $\quad$ iii

Table of Contents $\quad$ iv

List of Tables vii

List of Figures viii

1 Introduction 1

2 Neutrinos 3

2.1 The Conception and Discovery of Neutrinos . . . . . . . . . . 3

2.2 Properties of Neutrinos . . . . . . . . . . . . . . . . . 4

2.2.1 Neutrino Mass . . . . . . . . . . . . . . . . 5

2.3 Double-beta decay . . . . . . . . . . . . . . . . . . 7

2.4 Neutrinoless Double-Beta Decay Experiments . . . . . . . . . . . . . 11

$30 \nu \beta \beta$ Counting Experiments $\quad 14$

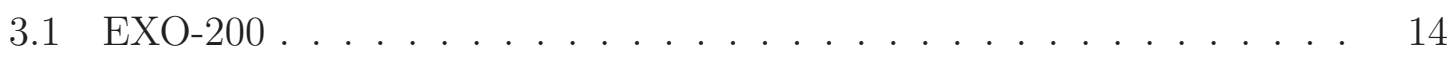

3.1.1 EXO-200 Detector .................. 15

3.1.2 Event Reconstruction . . . . . . . . . . . . . . 17

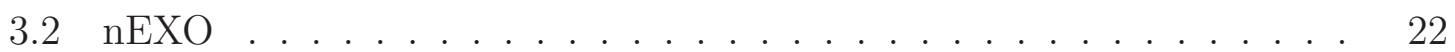

3.2.1 The nEXO Experiment . . . . . . . . . . . . . . 23

4 EXO-200 Analysis $\quad 24$

4.1 EXO-200 Previous Analysis . . . . . . . . . . . . . . . 24

4.1.1 EXO-200 Results .................. 26 
4.2 Boosted Decision Trees . . . . . . . . . . . . . . . . . 27

4.2.1 BDTs and Binary Decision Trees . . . . . . . . . . . 28

4.2 .2 BDTs in TMVA . . . . . . . . . . . . . . . . . . 32

4.2 .3 An Unbiased BDT . . . . . . . . . . . . . . . . . . . 35

4.3 EXO-200 Monte Carlo Simulation . . . . . . . . . . . . . . . 37

4.4 A $\beta$-like $/ \gamma$-like BDT Discriminator . . . . . . . . . . . . . . 39

4.5 Analysis Process . . . . . . . . . . . . . . . . . . . 49

4.5 .1 Sensitivity . . . . . . . . . . . . . . . . 55

$4.5 .2 \quad$ Discovery Potential . . . . . . . . . . . . . . . 57

4.5 .3 Systematic Uncertainty . . . . . . . . . . . . . . . . . 58

4.6 Results . . . . . . . . . . . . . . . . . . . . . . . . . . . 60

5 Ba Tagging $\quad 63$

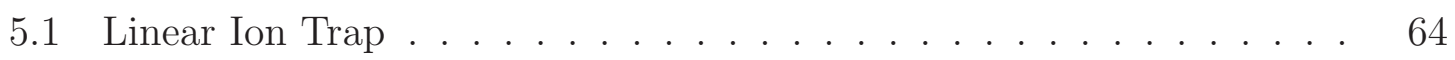

5.1 .1 The Adiabatic Approximation . . . . . . . . . . . . . 65

$5.1 .2 \quad$ Linear Quadrupole Mass Filters . . . . . . . . . . . . . . . 68

5.1.3 The Second Generation Stanford Linear Quadrupole Ion Trap 70

5.2 Optical Tagging . . . . . . . . . . . . . . . . . . . . 75

5.2 .1 The Ba-II System . . . . . . . . . . . . . . . . . . . 75

5.2 .2 The Optical Bloch Equations _. . . . . . . . . . . 78

$5.2 .3 \mathrm{Ba}^{+}$Natural Linewidth and the Doppler Shift . . . . . . . 82

5.2 .4 Experimental Setup . . . . . . . . . . . . . . . . . . 87

5.3 Measurement Technique and Old Results . . . . . . . . . . . . 94

5.3 .1 Laser Ablation Ion Source . . . . . . . . . . . . . . . . . . . 94

5.3 .2 Laser Alignment . . . . . . . . . . . . . . . . . . . . 95

5.3 .3 Measurement Techniques . . . . . . . . . . . . . . . . . . 95

5.3 .4 Ion Counting Results . . . . . . . . . . . . . . . . . . . . . 99

5.4 Improvements to system $\ldots \ldots \ldots \ldots \ldots$

5.4.1 Anti-reflective Black Coating . . . . . . . . . . . . . . . 101

5.4 .2 Optics Improvements . . . . . . . . . . . . . . . . . . 103

5.4 .3 Improved Results . . . . . . . . . . . . . . . . . . 103

5.5 Isotope Tagging . . . . . . . . . . . . . . . . . . . 106

$\begin{array}{lll}6 & \text { Conclusion } & 108\end{array}$ 
List of References 


\section{List of Tables}

2.1 Experimental values of the neutrino mixing parameters separated by mass hierarchy ("Both" denotes values which are the same for both hierarchies). . . . . . . . . . . . . . . . 7

2.2 Decay parameters of various $\beta \beta$-decay candidate isotopes. The nuclear matrix element, $M^{0 \nu}$ is presented using two different methods of calculation, the IBM-2 method and the QRPA method. . . . . . . . . 12

2.3 The lower limits on the $0 \nu \beta \beta$ half-lifetime imposed by KamLAND-Zen, Gerda and EXO-200. . . . . . . . . . . . . . . . . . . 13

3.1 Basic information on the radioactive sources used for detector calibration in EXO-200. . . . . . . . . . . . . . . . . . . . 20

3.2 EXO-200 effective energy resolution fit results. . . . . . . . . . . . . . 22

4.1 EXO-200 results published in Nature, 2014. . . . . . . . . . . . . . . 27

4.2 TMVA options used in training the BDT. . . . . . . . . . . . . 34

4.3 All components of the full EXO-200 PDF along with the corresponding fit result for their $n_{i}$ parameter. . . . . . . . . . . . . . . . 52

4.4 Results of EXO-200 analysis using full Phase-I dataset comparing the new 2D energy vs. BDT discriminator fit to the previously used energy vs. standoff distance fit as well as a 1D energy-only fit. . . . . . . . . 61

5.1 Operating parameters for the second generation ion trap. . . . . . . . 76

5.2 Vacuum wavelength $(\lambda)$, branching ratio, and spontaneous emission rate $(\Gamma)$ for energy level transitions in the Ba-II system. . . . . . . . . 83

5.3 Specifications of the Hamamatsu PMT used for light collection. . . . 93

5.4 Some physical properties of Acktar Magic Black ${ }^{\mathrm{TM}}$. . . . . . . . . . . 102 


\section{List of Figures}

2.1 The effective Majorana mass vs. the lightest neutrino state mass. The three neutrino mass hierarchies are indicated. . . . . . . . . . . . 8

2.2 The Feynman diagram of neutrinoless double-beta decay. Majorana neutrinos from two simultaneous beta decays annihilate, violating lepton number conservation. . . . . . . . . . . . . . . . . .

2.3 The energy spectra of $2 \nu \beta \beta$ and $0 \nu \beta \beta$. The main plot greatly exaggerates the height of the $0 \nu$ peak for the sake of visibility whereas the inset plot shows a more realistic height. Both distributions have been smeared to account for the resolution limitations of a realistic detector. 11

3.1 A schematic of the EXO-200 TPC vessel. . . . . . . . . . . . . . . . 15

3.2 A cut-away view of the clean room module at the Waste Isolation Pilot Plant containing the EXO-200 detector. . . . . . . . . . . . . . 16

3.3 Diagram showing the orientation of the $\mathrm{U}-\mathrm{V}$ and $\mathrm{X}-\mathrm{Y}$ coordinate systems as well as the fiducial and active regions of the Xe TPC. . . . . 18

3.4 Diagram of the calibration source locations in the EXO-200 TPC. The $\mathrm{X}-\mathrm{Y}-\mathrm{Z}$ coordinates of each are as follows: $\mathrm{S} 2=(0,0,-29.5 \mathrm{~cm}), \mathrm{S} 5=$ $(25.5 \mathrm{~cm}, 0,0), \mathrm{S} 8=(0,0,29.5 \mathrm{~cm}), \mathrm{S} 11=(0,25.5 \mathrm{~cm}, 0)$. In addition, $\mathrm{S} 17=(0,-25.5 \mathrm{~cm}, 0)$ is not shown. . . . . . . . . . . . . 20

3.5 Scintillation energy vs. ionization energy for the ${ }^{228}$ Th source at S5. The minimization of energy resolution by rotation is illustrated here.

4.1 A 1D projection of the fit into energy are shown. Figure 4.1(a) shows the result for SS events and figure 4.1(b) shows the result for MS events. 25 
4.2 The NLL profile for $0 \nu \beta \beta$ counts. The negative-log-likelihood $(\lambda)$ is shown on the y-axis (offset to achieve minimum value of 0 ) vs. the number of $0 \nu$ events on the x-axis. The dashed green (black) line represent the $90 \%(1 \sigma)$ confidence level upper limit on the number of $0 \nu$ events. The minimum near 10 indicates the central value of the fit

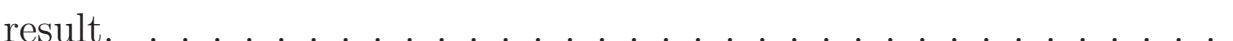

4.3 Figure 4.3(a) depicts the decision process used by a simple binary decision tree with two input variables. Figure 4.3(b) shows the regions in 2D parameter space of either signal-like or background-like events defined by the decision tree. . . . . . . . . . . . . . . . . . . . 28

4.4 The process by which a binary decision tree is trained. . . . . . . . . 30

4.5 Figure 4.5(a) shows the process by which a BDT calculates its classifier score from the weighted average of binary trees. Here $\mathrm{w}_{1,2,3}$ are the weights of the binary trees, $t_{1,2,3}$. Figure $4.5(\mathrm{~b})$ depicts the $2 \mathrm{D}$ parameter space of a BDT with three binary trees. There are now regions corresponding to different classifier scores rather than regions of signal or background. . . . . . . . . . . . . . . . . . . . . . 31

4.6 The process by which a boosted decision tree is trained. . . . . . . . . 33

4.7 Simulated EXO-200 TPC geometry in GEANT4, showing the TPC vessel and and copper internal components. . . . . . . . . . . . . 38

4.8 Standoff distance shape agreement showing event distributions (upper) and residuals (lower) for both ${ }^{228} \mathrm{Th}$ and $2 \nu \beta \beta$ events. Here a nonuniform binning is used in which we expect signal-like events to be near-uniformly distributed. . . . . . . . . . . . . . . . .

4.9 Standoff distance distributions for $0 \nu \beta \beta, 2 \nu \beta \beta$ and background events with calculated separation scores between each. . . . . . . . . . . . . 41

4.10 Figure 4.10(a) shows a U-wire waveform and figure 4.10(b) shows the matched filter result and corresponding pulse threshold. . . . . . . . . 42

$4.11 \mathrm{U}$ - and $\mathrm{V}$-wire waveforms fit to their corresponding signal models. . .

4.12 Cluster risetime shape agreement showing event distributions (upper) and residuals (lower) for both ${ }^{228} \mathrm{Th}$ and $2 \nu \beta \beta$ events. Red vertical lines indicate the upper and lower risetime profiling thresholds. . . . .

4.13 Cluster risetime distributions for $0 \nu \beta \beta, 2 \nu \beta \beta$ and background events with calculated separation scores between each. . . . . . . . . . 
4.14 Collection wire shape agreement showing event distributions (upper) and residuals (lower) for both ${ }^{228} \mathrm{Th}$ and $2 \nu \beta \beta$ events. . . . . . . . . . 45

4.15 Collection wire distributions for $0 \nu \beta \beta, 2 \nu \beta \beta$ and background events with calculated separation scores between each. . . . . . . . . . . 46

4.16 BDT discriminator shape agreement showing event distributions (upper) and residuals (lower) for both ${ }^{228} \mathrm{Th}$ and $2 \nu \beta \beta$ events. . . . . . .

4.17 BDT discriminator distributions for $0 \nu \beta \beta, 2 \nu \beta \beta$ and background events with calculated separation scores between each. . . . . . . .

4.18 Fit result using the new analysis technique showing 1-D projections into energy for SS (with ROI inset) and MS events (only ROI shown) and BDT discriminator for SS only. Results are shown for fit to full Phase-I data set. Background components in the fit are grouped according to their colors in table 4.3. . . . . . . . . . . . . . .

4.19 The NLL profile for the number of $0 \nu \beta \beta$ events. Profiles are shown for the Energy + BDT fit detailed in this work as well as the baseline Energy + Standoff Distance fit and a 1D Energy only fit. The $90 \%$ confidence level (assuming the validity of Wilk's theorem) is indicated by the dashed horizontal green line. . . . . . . . . . . . . . . .

4.20 A histogram of the $90 \%$ CL upper limits on the number of $0 \nu \beta \beta$ events calculated on 5000 toy data sets. The vertical red line indicates the median upper limit corresponding to the sensitivity of the experiment

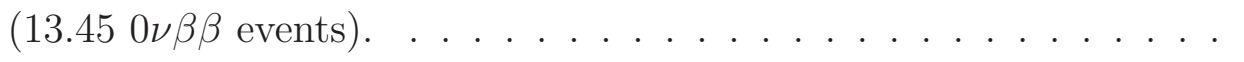

4.21 The distribution of the test statistic, $t$, for the null hypothesis and an alternate hypothesis

5.1 A diagram of the planned barium tagging scheme. A barium ion is extracted from the liquid xenon TPC via a cryogenic probe at which point it is evaporated toward the entrance of an RF funnel. This funnel then removes any gaseous xenon allowing the ion to be trapped and optically tagged in vacuum. Also shown is an MR-TOF system which will act as a secondary detection after the optical tagging. Logos are shown for the various instutions at which each component is being

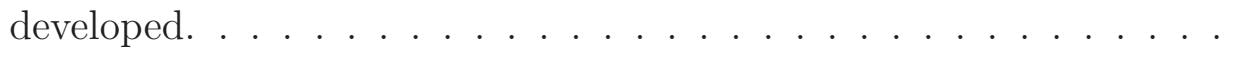

5.2 Cross-section of the electrode layout of a typical linear quadrupole. Neighbouring rods carry RF voltage of opposite sign. . . . . . . . . . 66 
5.3 Region of stable ion trajectory bounded by $\beta_{\mathrm{u}}=0,1 . . . .$. .

5.4 The longitudinal potential is shown along the centre $(r=0 \mathrm{~mm}$ of the trap using DC offset potentials found in table 5.1. This potential was calculated using the SIMION ion flight simulation software. Below, the segmentation of the ion trap is illustrated. . . . . . . . . . . .

5.5 A CAD model of the ion trap is shown here depicting the trap electrodes, macor mounting block and steel support frace. Figure 5.5(a) shows the front face of the ion trap. Figure 5.5(b) shows the ion trap from the top, focusing on the trapping region. Figure 5.5(c) shows an isometric view of the ion trap assembly. . . . . . . . . . . . . . . . 72

5.6 A single RF/DC mixing circuit used combine the quadrupole RF potentials with the individual segment DC offset voltages. . . . . . . .

5.7 Some photos of the assembled ion trap. Figure 5.7(a) shows the entrance of the trap. Figure 5.7(b) shows the trapping region as seen through one of the vacuum chamber viewports. Some of the wiring is visible in both photos which carries the mixed DC and RF voltages to the rods. . . . . . . . . . . . . . . . . . . . 73

5.8 A schematic of the buffer gas supply system to the ion trap. . . . . . 74

5.9 The three energy levels of the Ba-II system with lifetimes and branching ratios labelled as well as the wavelengths of dipole transition photons. 77

5.10 Excited state population, $\rho_{a a}$ as a function of $\delta_{\mathrm{B}}$ and $\delta_{\mathrm{R}}$. Here $\Omega_{\mathrm{B}}=$ $2 \pi(10 \mathrm{MHz})$ and $\Omega_{\mathrm{R}}=2 \pi(10 \mathrm{MHz})$. . . . . . . . . . . .

5.11 Excited state population, $\rho_{a a}$ as a function of $\delta_{\mathrm{B}}$ for different values of $\delta_{\mathrm{R}}$. Here $\Omega_{\mathrm{B}}=2 \pi(10 \mathrm{MHz})$ and $\Omega_{\mathrm{R}}=2 \pi(10 \mathrm{MHz}) . \ldots \ldots$

5.12 Excited state population, $\rho_{a a}$ as a function of $\delta_{\mathrm{B}}$ for different values of $\Omega_{\mathrm{B}}$. Here $\delta_{\mathrm{R}}=0 \mathrm{MHz}$ and $\Omega_{\mathrm{R}}=2 \pi(10 \mathrm{MHz}) \ldots . . . . .$.

5.13 Excited state population, $\rho_{a a}$, with Doppler broadening at $298 \mathrm{~K}$ as a function of $\delta_{\mathrm{B}}$ for different values of $\delta_{\mathrm{R}}$. Here $\Omega_{\mathrm{B}}=2 \pi(10 \mathrm{MHz})$ and $\Omega_{\mathrm{R}}=2 \pi(10 \mathrm{MHz}) \ldots \ldots \ldots \ldots \ldots \ldots$

5.14 Doppler broadened linewidths for ${ }^{136} \mathrm{Ba}$ and ${ }^{138} \mathrm{Ba}$. Also shown is the combined linewidth when one of each isotope is present. Here $\Omega_{\mathrm{B}}=$ $2 \pi(10 \mathrm{MHz}), \Omega_{\mathrm{R}}=2 \pi(10 \mathrm{MHz})$ and $\delta_{\mathrm{R}}^{136}=0 \mathrm{MHz} \ldots \ldots . .$. 
5.15 The components of a TOPTICA TA/SHG pro laser (the blue laser). In the case of a TA pro (the red laser), the beam output is after the second optical isolator and the rest of the components do not exist. .

5.16 A schematic of the beam paths from the laser outputs to the ion trap.

5.17 Beam profiles of the blue and red lasers after shaping optics. Profiles were measured using a Newport LBP beam profiler. . . . . . . . . . .

5.18 Red and blue laser duty cycles during continuous excitation counting technique. The blue laser is held on continuously and the red laser is pulsed on and off with a $50 \%$ duty cycle. . . . . . . . . . . . .

5.19 A single MCS scan using the continuous excitation measurement technique. Each bin constitutes $0.02 \mathrm{~s}$. In the first half of the scan, the red laser is turned off and there is only background scattered light. In the second half, the red laser is turned on and signal is observed. . . . . .

5.20 Red and blue laser duty cycles during the intermodulation counting technique. The blue laser is held on for the first $60 \%$ of the cycle, it is then switched off and the red laser is turned on for the last $6 \%$. . .

5.21 A single MCS scan using the intermodulation measurement technique. Each bin spans $0.02 \mathrm{~s}$. The first 30 bins show scattered light while the blue laser is on. The following 17 bins show a gradual decrease is signal as the PMT cools, and the final 3 bins show signal while the red laser

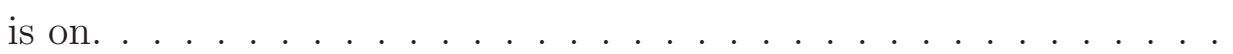

5.22 Previous barium tagging results. Discrete steps in photon count rate indicate individual ions exiting the trap. Figure 5.22(a) shows a SNR of $\sim 4.6$ and $\mathrm{S} / \mathrm{B}$ of $\sim 0.16$. Figure $5.22(\mathrm{~b})$ shows a SNR of $\sim 8.8$ and

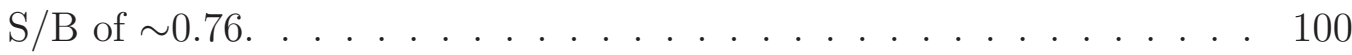

5.23 The hemispherical reflectance of Acktar Magic Black ${ }^{\mathrm{TM}}$ and Vacuum Black $^{\mathrm{TM}}$, another ultra-black coating which was considered. . . . . . . 101

5.24 Photograph of trap electrodes coated with Acktar Magic Black ${ }^{\mathrm{TM}}$. Some rods show minor damage to the coating due to handling during installation. . . . . . . . . . . . . . . . . . 
5.25 Discrete ion steps found using the intermodulation technique after improvements to background reduction in the ion trap. The photon rate of each ion step and their corresponding noise are found by fitting the photon count rates of each step to a gaussian function (shown on the right). This scan shows a SNR of 5.6 and S/B of 1.2. . . . . . . . . . 104

5.26 New ion counting attempt using the continuous excitation method. There are hints of discrete ion steps, however still not as good as the intermodulation method. . . . . . . . . . . . . . . . . . 105

5.27 Figure 5.27(a) shows the design of a new bladed quadrupole ion trap and figure 5.27(b) shows the design of a similar trap used at Innsbruck upon which the new design was based. This new bladed design is in the design state at TRIUMF and is intended to replace Carleton's ion trap for use in barium tagging when completed. . . . . . . . . . . . 105

5.28 An experimentally observed $\mathrm{Ba}^{+}$linewidth with many ions present. This was observed by measuring the fluorescence signal while varying the frequency setpoint of the blue laser. The value of $\delta_{\mathrm{B}}$ here is the detuning from resonance for ${ }^{136} \mathrm{Ba}$. . . . . . . . . . . . . . . 107 


\section{Chapter 1}

\section{Introduction}

Neutrinos have been known in the world of physics for nearly 100 years now, but there is still much to be learned about them. Only recently it has been shown by neutrino oscillation experiments such as SNO [1] and Super-K [2] that neutrinos carry mass, a fact inconsistent with the Standard Model of particle physics and in fact the first experimental evidence of physics Beyond the Standard Model (BSM). While the mass splitting between the three neutrino flavors has been measured, as of yet the absolute mass scale remains unknown, a fact which has led to a slew of new physics experiments intent on making a mass measurement.

The current leading experiments in the search for the neutrino mass scale are all searches for neutrinoless double-beta decay $(0 \nu \beta \beta)$, one of which is the Enriched Xenon Observatory (EXO). The EXO collaboration is searching for $0 \nu \beta \beta$ in liquid ${ }^{136}$ Xe using a $200 \mathrm{~kg}$ time projection chamber (EXO-200). Up until recently, EXO200 held the leading result for the $0 \nu \beta \beta$ half-life lower limit in ${ }^{136} \mathrm{Xe}$ and now aims to publish a new result in the near future. The primary focus of this work is on the development of an improved analysis technique which will be used by EXO-200 in this upcoming publication.

In addition to EXO-200, the EXO collaboration has also begun development of a successor experiment, a 5 tonne liquid xenon TPC dubbed nEXO (next EXO). As an upgrade to the detector, nEXO will eventually add systems to utilize a technique called barium tagging, in which the ${ }^{136} \mathrm{Ba}^{++}$daughter ion of the double-beta decay process is identified in order to remove any non- $\beta \beta$ backgrounds. While significant effort has already been made toward developing a system for barium tagging [3], this work implements improvements to the sensitivity of this existing system.

This thesis has the following structure. Chapter 2 presents the history of neutrinos 
from their conception to the neutrino oscillation experiments which proved they have mass. This chapter also explains the theory behind neutrino oscillations, doublebeta decay (both the two-neutrino and neutrinoless flavors) and their importance in measuring the absolute neutrino mass scale.

Chapter 3 describes the two major $0 \nu \beta \beta$ experiments which are the focus of this work, EXO-200 and nEXO. A description is given of the EXO-200 detector hardware as well as the process of event reconstruction. The nEXO detector is introduced focusing on the differences between it and its predecessor.

Chapter 4 then explains techniques used in analysis of EXO-200 physics data, beginning from the technique used in previous publications and presenting the most recent results, and continuing on to cover the newly developed analysis technique and presenting some preliminary new results. The explanation of the new analysis technique begins by introducing the concept of Multivariate Analysis (MVA) and the chosen technique (boosted decision trees, or BDTs). A full description of BDTs is then given covering conceptually how they function, and how they are built. The configuration of the BDT used in this analysis is then given including detailed descriptions of all BDT input variables. The chapter then continues on to describe the calculation of the $0 \nu \beta \beta$ half-life lower limit (and consequently the effective Majorana-neutrino mass upper limit), the experimental sensitivity and the discovery potential. It then concludes by presenting the preliminary results calculated using this new analysis on the full EXO-200 Phase-I data set.

Finally, chapter 5 describes the barium tagging system including the theory behind it, the experimental setup and the recent improvements. The chapter begins with an explanation of RF ion trapping theory and a description of the Stanford second generation linear ion trap in use at Carleton. It then presents the theory behind ion detection via laser induced fluorescence of the Ba-II system followed by a description of the laser systems used for this purpose. The chapter then goes on to show the results of previous barium tagging work, followed by the improvements to the system and new results.

The bulk of the original work of this thesis comprises chapter 4, in which the new analysis technique for EXO-200 is developed, and section 5.4 in which upgrades are made to the existing barium tagging apparatus leading to new single ion measurements. Some additional original work is shown in section 5.5 where the use of the barium tagging system to tag individual barium isotopes is explored. 


\section{Chapter 2}

\section{Neutrinos}

While the neutrino was proposed nearly a hundred years ago it remains quite mysterious to this day. It has only recently been shown thanks to experiments such as the Sudbury Neutrino Observatory (SNO) and Super Kamiokande (Super-K) that neutrinos undergo flavor oscillations and thus have non-zero mass. Despite the fact that they are known to have mass, this exact value has not yet been measured and many experiments to this effect are now underway worldwide by various experimental collaborations. Given that this work is a part of the search efforts of the Enriched Xenon Observatory experiments, EXO-200 and nEXO, it seems fitting that I first explore the history of the neutrino, beginning from it's conception as a solution to the non-conservation of energy in $\beta$-decay.

\subsection{The Conception and Discovery of Neutrinos}

In the early $20^{\text {th }}$ century, the current model of the atomic nucleus had not yet been established. At the time, the only known subatomic particles were the proton, electron and photon. The process of beta decay was thus understood to be,

$$
X \rightarrow Y^{+}+\mathrm{e}^{-}
$$

where a nucleus, $X$, is converted to a nucleus of lower mass $Y$ via the release of an

electron, e. It was expected, due to conservation of energy, that the electron would be emitted with the kinetic energy equivalent of the mass difference between $X$ and $Y$ however in 1927, C.D. Ellis and W. Wooster published the results of a study in which they showed a continuous energy spectrum from beta decay [4], a fact which seemed 
to imply non-conservation of energy. In 1930, as a solution to the problem termed the "energy crisis", Wolfgang Pauli proposed the existence of a new particle, the "neutron", which existed within the nucleus and was emitted in the $\beta$-decay process, thus accounting for the lost energy.

While Pauli's "neutron" was eventually renamed due to the 1932 discovery of the modern day neutron by James Chadwick [5], the concept of an extra particle to account for the loss of energy was sound. In 1934, Enrico Fermi published a paper on $\beta$-decay in which he established the theory behind neutrinos and their part in the beta decay process [6]. This new understanding of the process described beta decay as the conversion of a neutron, $\mathrm{n}$, into a proton, $\mathrm{p}$, via the release of an electron and a neutrino (specifically the electron anti-neutrino), $\bar{\nu}_{\mathrm{e}}$,

$$
\mathrm{n} \rightarrow \mathrm{p}^{+}+\mathrm{e}^{-}+\bar{\nu}_{\mathrm{e}}
$$

By examining the shape of the beta decay energy spectrum Fermi was able to predict that the neutrino must have a mass much smaller than that of the electron (possibly 0). Furthermore, Fermi's theory implied that a similar process to $\beta$ decay should occur, called inverse beta decay, in which a proton is converted into a neutron via the capture of a neutrino and the release of an anti-electron (or positron),

$$
\bar{\nu}_{\mathrm{e}}+\mathrm{p}^{+} \rightarrow \mathrm{n}+\mathrm{e}^{+}
$$

This process was finally observed in 1956 by Reines and Cowan [7] using a liquid scintillator and the neutrino flux from the core of the Savannah River, South Carolina, nuclear reactor, thus proving the existence of neutrinos.

\section{$2.2 \quad$ Properties of Neutrinos}

Ever since the discovery of neutrinos, the measurement of their various properties has been a popular subject for experiments in the field of particle physics. In 1958, it was shown at Brookhaven National Laboratory (BNL) that neutrinos are left-handed [8] (i.e. the direction of its spin is opposite to that of its momentum). Again at BNL, it was discovered that neutrinos come in different flavors, one for each lepton (at the time only the electron and muon were known). Later, with the discovery of the tau

lepton, a third neutrino flavor was expected. This third flavor was discovered by the 
DONUT experiment in 2001 [9].

\subsubsection{Neutrino Mass}

The neutrino mass remains to this day unknown and is of great interest in the realm of particle physics. Although the standard model of particle physics does not attribute mass to the neutrino, we now know from observation of flavor oscillations that at least two neutrino flavors must carry mass. Unfortunately, because the neutrino interacts so weakly with regular matter, conventional techniques for measuring the mass (such as measurement of the energy spectrum of $\beta$-decay) have so far only managed to set upper limits and have not achieved a successful mass measurement.

The concept of neutrino flavor oscillations was first proposed by Bruno Pontecorvo in 1968 [10] to explain the "solar neutrino problem", in which the measured flux of solar neutrinos was significantly lower than that predicted by theory. By undergoing flavor oscillations, the solar electron neutrinos could become muon or tau neutrinos explaining the shortage. This idea was not without precedent since particle oscillation had already been observed in the neutral kaon system in 1956 by Leon Lederman [11]. Neutrino flavor oscillations were first observed by Super-K in 1998 [2] and then more definitively by SNO in 2001 [1] leading to a Nobel Prize shared by members of both collaborations.

While the observation of flavor oscillations proves that neutrinos are in fact massive, it does not tell us exactly what their mass is. In order to understand the search for the neutrino mass, it will be useful to first examine the physics behind flavor oscillations.

To begin, we define the neutrino flavor eigenstates as $\left(\nu_{\mathrm{e}}, \nu_{\mu}, \nu_{\tau}\right)$ and the mass eigenstates as $\left(\nu_{1}, \nu_{2}, \nu_{3}\right)$. In order for flavor oscillations to occur, it is necessary that

there be a difference between these two sets of states. The two sets of eigenstates are related to each other via,

$$
\nu_{\alpha}=\sum U_{\alpha, i}^{*} \nu_{i}
$$

where $U_{\alpha, i}^{*}$ is a PMNS (Pontecorve-Maki-Nakagawa-Sakata) matrix element. Using the notation that $c_{i j}=\cos \theta_{i j}$ and similarly $s_{i j}=\sin \theta_{i j}$, where $\theta_{i j}$ are mixing angles, the PMNS matrix is given by, 


$$
U_{\mathrm{PMNS}}=\left[\begin{array}{ccc}
c_{12} c_{13} e^{\frac{i \phi_{1}}{2}} & c_{13} s_{12} & s_{13} e^{-i \delta} \\
-s_{12} c_{23}-c_{12} s_{23} s_{13} e^{i \delta} & \left(c_{12} c_{23}-s_{12} s_{23} s_{13} e^{i \delta}\right) e^{\frac{i \phi_{2}}{2}} & s_{23} c_{13} \\
s_{12} s_{23}-c_{12} c_{23} s_{13} e^{i \delta} & -c_{12} s_{23}-s_{12} c_{23} s_{13} e^{i \delta} & c_{23} c_{13}
\end{array}\right]
$$

where $\delta$ is a CP-violating phase and $\phi_{i}$ are a pair of additional CP-violating phases which depend on the Majorana/Dirac nature of neutrinos. If neutrinos are Dirac particles, the two phases vanish, otherwise if neutrinos are Majorana then they may take non-zero values. Dirac particles have distinct particles and anti-particles, whereas a Majorana particle is it's own antiparticle. Currently every standard model lepton is understood to be Dirac except for the neutrinos whose natures have not yet been determined.

Flavor oscillations also depend on $\Delta m_{21}^{2}$ and $\Delta m_{32}^{2}$ (and $\Delta m_{31}^{2}$ however this is simply a linear combination of the other two) which are the square-mass differences between the mass eigenstates given by,

$$
\Delta m_{i j}^{2}=\Delta m_{i}^{2}-\Delta m_{j}^{2}
$$

where $\Delta m_{i}$ is the mass difference between the $i$-th neutrino mass eigenstate and the lightest eigenstate (and similarly for $\Delta m_{j}$ ). Neutrino oscillation experiments have been able to constrain the neutrino mass parameter-space. However, since they are unable to determine the sign of $\Delta m_{31}^{2}$ they cannot determine the absolute neutrino mass scale, hence the continued need for neutrino mass experiments. The best estimates of mixing parameters are shown in table 2.1, separated according to their associated mass hierarchy, a concept which will be explained shortly.

It is in part the measurement of $\Delta m_{21}^{2}$ that leads to the distinction of the masses into two hierarchies. Because $\Delta m_{21}^{2}$ was found to be positive it is implied that $m_{\nu_{2}}>$ $m_{\nu_{1}}$. This measurement restricts the options for ordering the neutrino masses into two possibilities, the normal hierarchy, where $m_{\nu_{3}}>m_{\nu_{2}}>m_{\nu_{1}}$, and the inverted hierarchy, where $m_{\nu_{2}}>m_{\nu_{1}}>m_{\nu_{3}}$. There is also a third hierarchy in which the mass splittings are negligible in comparison to the absolute mass scale in which case the three neutrino masses are treated as essentially equal and the normal and inverted hierarchies are indistinguishable. This occurs when the mass scale is on the order 
Table 2.1: Experimental values of the neutrino mixing parameters separated by mass hierarchy ("Both" denotes values which are the same for both hierarchies).

\begin{tabular}{|c|c|c|}
\hline Parameter & Hierarchy & Value \\
\hline \hline$\Delta m_{21}^{2}\left(\mathrm{eV}^{2}\right)$ & Both & $7.54_{-0.22}^{+0.26} \times 10^{-5}$ \\
$\left|\Delta m_{31}^{2}-\frac{1}{2} \Delta m_{21}^{2}\right|\left(\mathrm{eV}^{2}\right)$ & Normal & $2.43 \pm 0.06 \times 10^{-3}$ \\
$\left|\Delta m_{31}^{2}+\frac{1}{2} \Delta m_{21}^{2}\right|\left(\mathrm{eV}^{2}\right)$ & Inverted & $2.38 \pm 0.06 \times 10^{-3}$ \\
$\sin ^{2} \theta_{12}$ & Both & $0.308 \pm 0.017$ \\
$\sin ^{2} \theta_{23}$ & Normal & $0.437_{-0.023}^{+0.033}$ \\
& Inverted & $0.455_{-0.031}^{+0.039}$ \\
$\sin ^{2} \theta_{13}$ & Normal & $0.234_{-0.0020}^{+0.0019}$ \\
& Inverted & $0.455_{-0.0019}^{+0.0022}$ \\
\hline
\end{tabular}

${ }^{*}$ Values from [12].

of $\sim 0.1 \mathrm{eV}$. The different hierarchies occupy different regions in the mass-parameter space (depicted in figure 2.1) and therefore a measurement of the neutrino mass scale would indicate which hierarchy the neutrino masses follow.

So far experiments have failed to measure the neutrino mass or determine the mass hierarchy, only managing to set upper limits. Some newer experiments however aim to exploit an exotic form of $\beta$-decay in order to measure the absolute neutrino mass scale.

\subsection{Double-beta decay}

The exotic double-beta decay is a simple extension from the more common beta decay in which two beta decays occur simultaneously. This decay comes in two flavors, twoneutrino double-beta decay $(2 \nu \beta \beta)$ and neutrinoless double-beta decay. While the former has been observed experimentally in a number of different isotopes, the latter has not and would be very informative if it were to be seen.

Neutrinoless double-beta decay is a similar decay process to two-neutrino doublebeta decay in which the two neutrinos annihilate together. This can also be interpreted as a single virtual neutrino taking part in both beta decays. A Feynman 


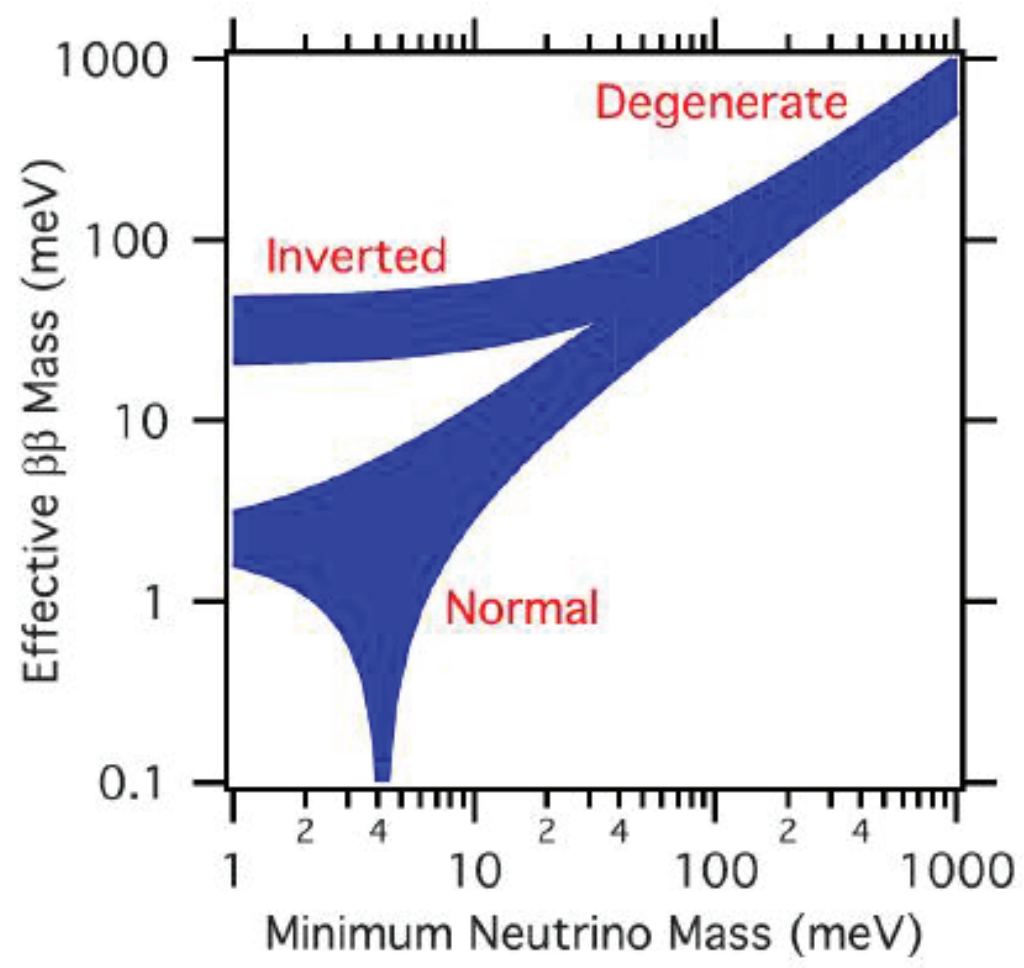

Figure 2.1: The effective Majorana mass vs. the lightest neutrino state mass. The three neutrino mass hierarchies are indicated. Reprinted from [13]. 


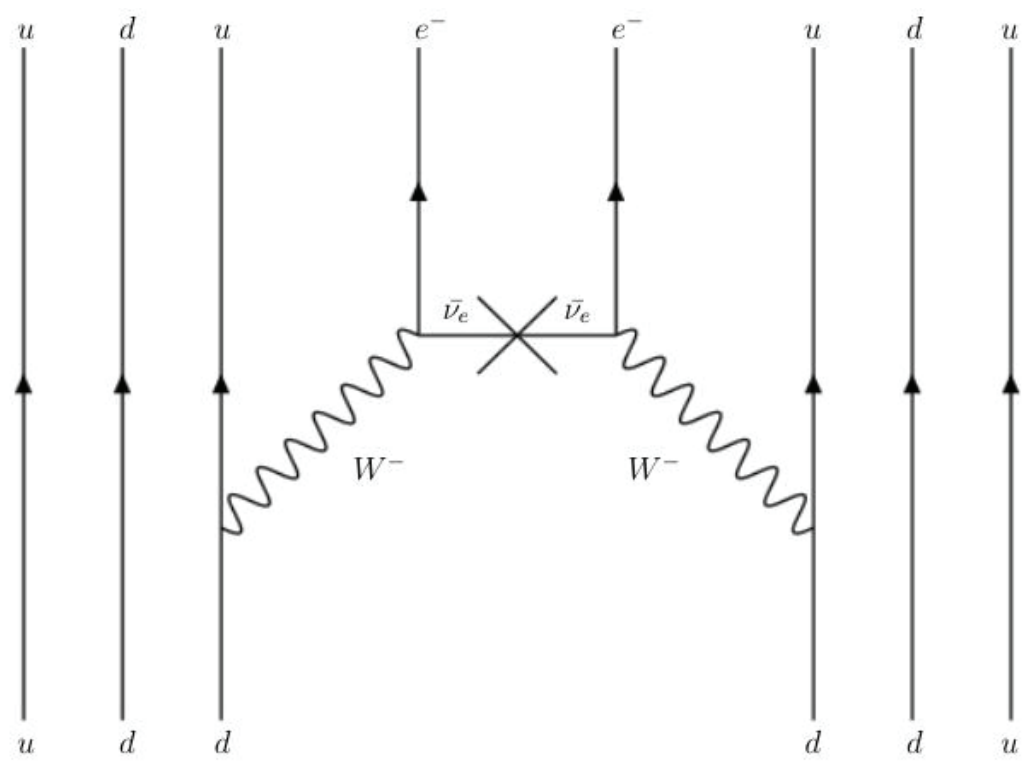

Figure 2.2: The Feynman diagram of neutrinoless double-beta decay. Majorana neutrinos from two simultaneous beta decays annihilate, violating lepton number conservation.

diagram of the decay can be seen in figure 2.2. This decay is of particular interest because it requires that neutrinos are Majorana in nature and it demonstrates violation of lepton number conservation, both properties which are not explicitly forbidden in the Standard Model but are not required by it and have yet to be observed. Furthermore, observation of this decay would lead to a measurement of Majorana mass scale of the neutrino.

There is one additional property of $0 \nu \beta \beta$ which leads toward a measurement of the neutrino mass scale. In order to understand this, the two beta decays must be considered separately. In the first decay, a right-handed neutrino is emitted, in the second a left-handed neutrino is absorbed. In order for this to be possible, the neutrino emitted from the first decay must have a left-handed component which is only possible if the neutrino is massive. Additionally, the amplitude of this lefthanded component (and consequently the rate at which $0 \nu \beta \beta$ occurs) is proportional to the mass of the neutrino. Therefore by measuring the rate of this decay, the mass of the neutrino can also be measured.

The half-life, $T_{1 / 2}^{0 \nu}$ of neutrinoless double-beta decay is given by [14], 


$$
\left[T_{1 / 2}^{0 \nu}\right]^{-1}=G^{0 \nu}\left(E_{0}, Z\right)\left[M^{0 \nu}\right]^{2} \frac{\left\langle m_{\beta \beta}\right\rangle^{2}}{m_{\mathrm{e}}^{2}}
$$

where $G^{0 \nu}$ is the leptonic phase space factor which can be calculated to any precision, $E_{0}$ is the total energy available in the decay, $Z$ is the nuclear charge number of the isotope undergoing the decay, $M^{0 \nu}$ is a nuclear matrix element (subject to a large theoretical error), $m_{\mathrm{e}}$ is the electron mass and $\left\langle m_{\beta \beta}\right\rangle$ is the effective Majorana mass. The Majorana mass term is a combination of the neutrino mass eigenstate masses given by,

$$
\left\langle m_{\beta \beta}\right\rangle=\sum U_{\mathrm{e}, i}^{2} m_{i}
$$

thus the neutrino mass scale can be measured.

In practice because it is difficult to detect neutrinos, $2 \nu$ and $0 \nu$ double-beta decays will look extremely similar when observed experimentally. This can potentially pose a problem since any isotope subject to either decay will be subject to both, guaranteeing a $2 \nu \beta \beta$ background in any attempt to observe $0 \nu \beta \beta$. Luckily the two decays can be differentiated via their energy spectra which will be significantly different. The $0 \nu \beta \beta$ energy spectrum is extremely simple. Since both electrons emitted from the decay can be observed, the sum of their energies must equal the Q-value of the decay (where the Q-value is defined as the sum energy of all electrons and neutrinos emitted from the decay) implying a $\delta$-function energy distribution. The spectrum for $2 \nu \beta \beta$ is more complicated. Because the two unobserved neutrinos carry off some of the decay energy, the electrons will only contain a fraction of the Q-value, following a continuous energy distribution between 0 and the Q-value. This distribution can be calculated using the Primakoff-Rosen approximation (given by [14]),

$$
\frac{d N_{2 \nu}}{d K} \sim K\left(T_{0}-K\right)^{5}\left(1+2 K+\frac{4 K^{2}}{3}+\frac{K^{3}}{3}+\frac{K^{4}}{30}\right)
$$

where $K$ is the sum energy of the two electrons and $T_{0}$ is the Q-value in units of electron mass. The energy spectra for $2 \nu$ and $0 \nu$ double-beta decays have been plotted in figure 2.3 with some energy smearing to approximate the resolution of a realistic detector. 


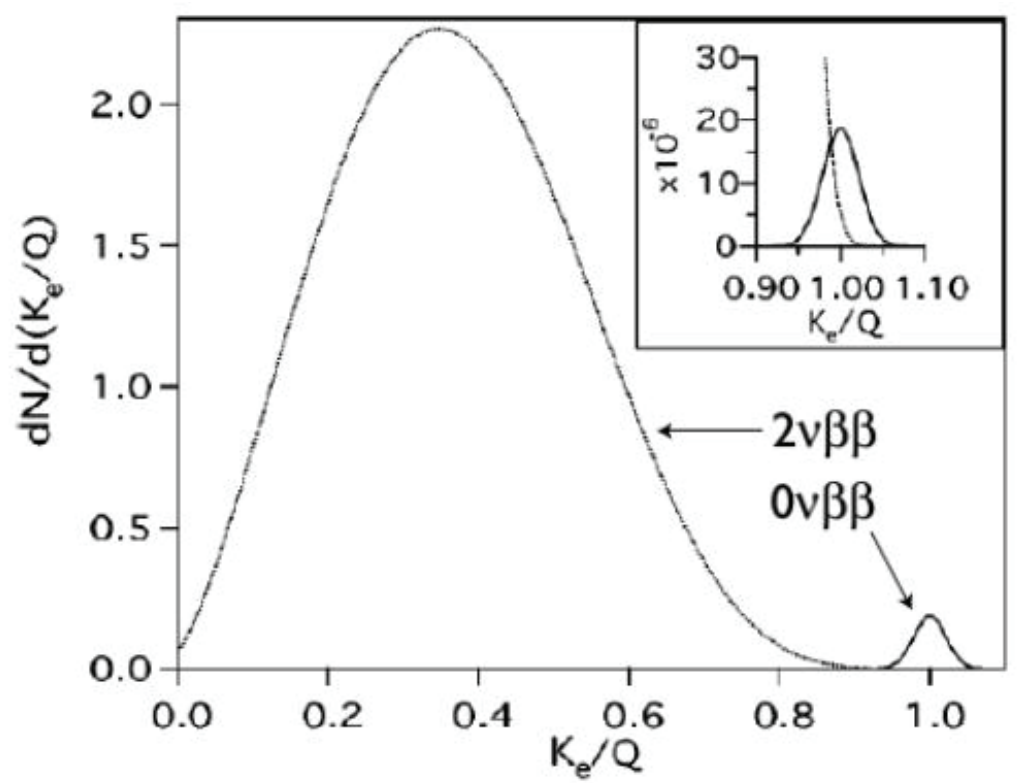

Figure 2.3: The energy spectra of $2 \nu \beta \beta$ and $0 \nu \beta \beta$. The main plot greatly exaggerates the height of the $0 \nu$ peak for the sake of visibility whereas the inset plot shows a more realistic height. Both distributions have been smeared to account for the resolution limitations of a realistic detector. Reprinted from [13].

\subsection{Neutrinoless Double-Beta Decay Experiments}

There are many candidate isotopes which may undergo double-beta decay with various different Q-values and lifetimes (summarized in table 2.2) and as such there are several experiments currently underway across the world searching for neutrinoless double-beta decay in various media. While none have yet successfully found $0 \nu \beta \beta$, they continue to push the upper mass limit lower and lower. Currently the best upper limit on the Majorana-neutrino mass is held by KamLAND-Zen [15], using the Kamioka Liquid Scintillator Antineutrino Detector in Toyama, Japan. Other competitive experiments include EXO-200 [16] (which will be discussed in depth later), GERDA [17] (Germanium Detector Array), at the Laboratori Nazionali del Gran Sasso in Italy and CUORICINO [18], also at the Gran Sasso facility. In addition to the currently active experiments there is also nEXO [19], the successor to EXO200 which will offer significantly improved sensitivity over its predecessor. All four of these experiments are passive counting experiments in which a volume of some chosen isotope is observed over a period of time to watch for neutrinoless double-beta decay. KamLAND-Zen, EXO-200 and nEXO are all liquid ${ }^{136}$ Xe based detectors whereas 
Table 2.2: Decay parameters of various $\beta \beta$-decay candidate isotopes. The nuclear matrix element, $M^{0 \nu}$ is presented using two different methods of calculation, the IBM-2 method and the QRPA method.

\begin{tabular}{|c|c|c|c|c|}
\hline Isotope & Q-value $(\mathrm{keV})$ & $G^{0 \nu}\left(10^{-15} \mathrm{yr}^{-1}\right)$ & \multicolumn{2}{|c|}{$M^{0 \nu}$ (dimensionless) } \\
\cline { 3 - 5 } & & & IBM-2 & QRPA \\
\hline \hline${ }^{48} \mathrm{Ca} \rightarrow{ }^{48} \mathrm{Ti}$ & 4272.26 & 64.34 & 2.82 & 0.871 \\
${ }^{76} \mathrm{Ge} \rightarrow{ }^{76} \mathrm{Se}$ & 2039.061 & 6.128 & 7.54 & 8.31 \\
${ }^{82} \mathrm{Se} \rightarrow{ }^{82} \mathrm{Kr}$ & 2995.12 & 26.35 & 6.01 & 7.47 \\
${ }^{96} \mathrm{Zr} \rightarrow{ }^{96} \mathrm{Mo}$ & 3350.37 & 53.37 & 4.56 & 4.38 \\
${ }^{100} \mathrm{Mo} \rightarrow{ }^{100} \mathrm{Ru}$ & 3034.4 & 41.28 & 6.80 & 8.70 \\
${ }^{110} \mathrm{Pd} \rightarrow{ }^{110} \mathrm{Cd}$ & 2017.85 & 12.49 & 6.52 & 9.28 \\
${ }^{116} \mathrm{Cd} \rightarrow{ }^{116} \mathrm{Sn}$ & 2813.5 & 43.31 & 4.99 & 6.51 \\
${ }^{124} \mathrm{Sn} \rightarrow{ }^{124} \mathrm{Te}$ & 2286.97 & 23.44 & 5.14 & 4.12 \\
${ }^{128} \mathrm{Te} \rightarrow{ }^{128} \mathrm{Xe}$ & 865.87 & 1.524 & 6.60 & 7.34 \\
${ }^{130} \mathrm{Te} \rightarrow{ }^{130} \mathrm{Xe}$ & 2526.97 & 36.88 & 5.96 & 6.26 \\
${ }^{136} \mathrm{Xe} \rightarrow{ }^{136} \mathrm{Ba}$ & 2457.83 & 37.81 & 4.91 & 3.51 \\
\hline
\end{tabular}

${ }^{*}$ Values from $[20-22]$.

GERDA uses ${ }^{76} \mathrm{Ge}$ crystal diodes and CUORICINO used ${ }^{130} \mathrm{Te}$ crystals. The most recent results of each experiment are presented in table 2.3. 
Table 2.3: The lower limits on the $0 \nu \beta \beta$ half-lifetime imposed by KamLAND-Zen, Gerda and EXO-200.

\begin{tabular}{|c|c|c|c|}
\hline Experiment & Medium & $T_{1 / 2}^{0 \nu}\left(\times 10^{25} \mathrm{yr}\right)$ & $\left\langle m_{\beta \beta}\right\rangle^{\dagger}(\mathrm{meV})$ \\
\hline \hline KamLAND-Zen & ${ }^{136} \mathrm{Xe}$ & $>10.7$ & $<61-165$ \\
EXO-200 & & $>1.1$ & $<190-450$ \\
GERDA & ${ }^{76} \mathrm{Ge}$ & $>5.3$ & $<150-330$ \\
CUORICINO & ${ }^{130} \mathrm{Te}$ & $>0.40$ & $<270-760$ \\
\hline
\end{tabular}

${ }^{\dagger}$ Results may vary based on the model used for the nuclear matrix element, $M^{0 \nu}$.

${ }^{*}$ Values from $[15-18]$. 


\section{Chapter 3}

\section{$0 \nu \beta \beta$ Counting Experiments}

The simplest method of searching for neutrinoless double beta decay $(0 \nu \beta \beta)$ is a passive search via a counting type experiment. A counting experiment operates by observing a medium in which the decay is expected to occur in order to measure the rate of decay. The Enriched Xenon Observatory (EXO) collaboration (technically split into two distinct collaborations, the EXO-200 and nEXO collaborations) currently has one operational prototype counting experiment (EXO-200) and another $(\mathrm{nEXO})$ in development. Both experiments search for $0 \nu \beta \beta$ in liquid ${ }^{136} \mathrm{Xe}$.

\subsection{EXO-200}

The EXO-200 experiment is a $\sim 200 \mathrm{~kg}$ liquid xenon (LXe) time projection chamber (TPC) originally built as a prototype by the EXO collaboration. It is located at the Waste Isolation Pilot Plant (WIPP) facility near Carlsbad, New Mexico. The experiment was originally planned as a prototype detector in preparation for a future ton-scale experiment.

EXO-200 operated in two phases. The first phase (Phase-I) collected data from 2011 until February 5, 2014 at which point the detector was shut down due to a set of unrelated incidents at the WIPP facility. In early 2016, the detector underwent upgrades to its electronics and a deradonator was installed to more closely monitor radon levels. After these upgrades, Phase-II data collection began in April 2016 and is currently ongoing. 


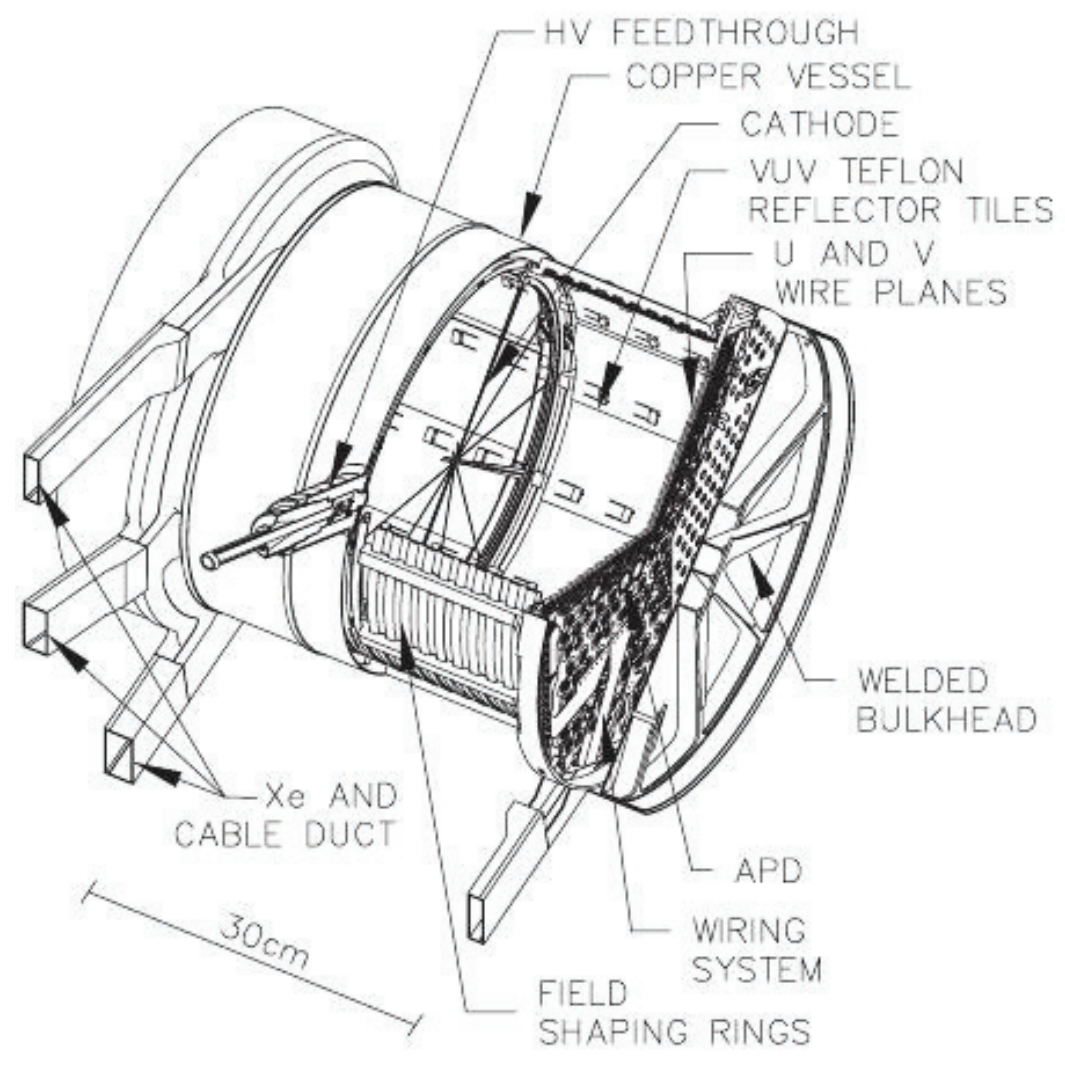

Figure 3.1: A schematic of the EXO-200 TPC vessel. Reprinted from [23].

\subsubsection{EXO-200 Detector}

The EXO-200 detector consists mainly of a time projection chamber filled with liquid xenon (enriched to $80.67 \pm 0.14 \%{ }^{136} \mathrm{Xe}$ ). The TPC itself consists of a cylindrical copper vessel ( $\sim 44 \mathrm{~cm}$ long with $\sim 40 \mathrm{~cm}$ diameter) which is divided in the centre by a cathode. At its end, the TPC is capped by identical sets of detection hardware; two wire planes, at $60^{\circ}$ from each other and a plane of avalanche photo-diode (APD) clusters. The first wire plane (closest to the cathode) is called the shielding plane, the second plane, called the collection plane, is located $6 \mathrm{~mm}$ behind the first with the APD plane another $6 \mathrm{~mm}$ behind that. With the cathode splitting the vessel in the middle, the detector is actually treated as two back-to-back TPCs with the collection wire plane acting as the anode in each (both TPCs are identical so in describing the operation of the TPCs only one will be explained). A schematic of the TPC vessel can be seen in figure 3.1 . 


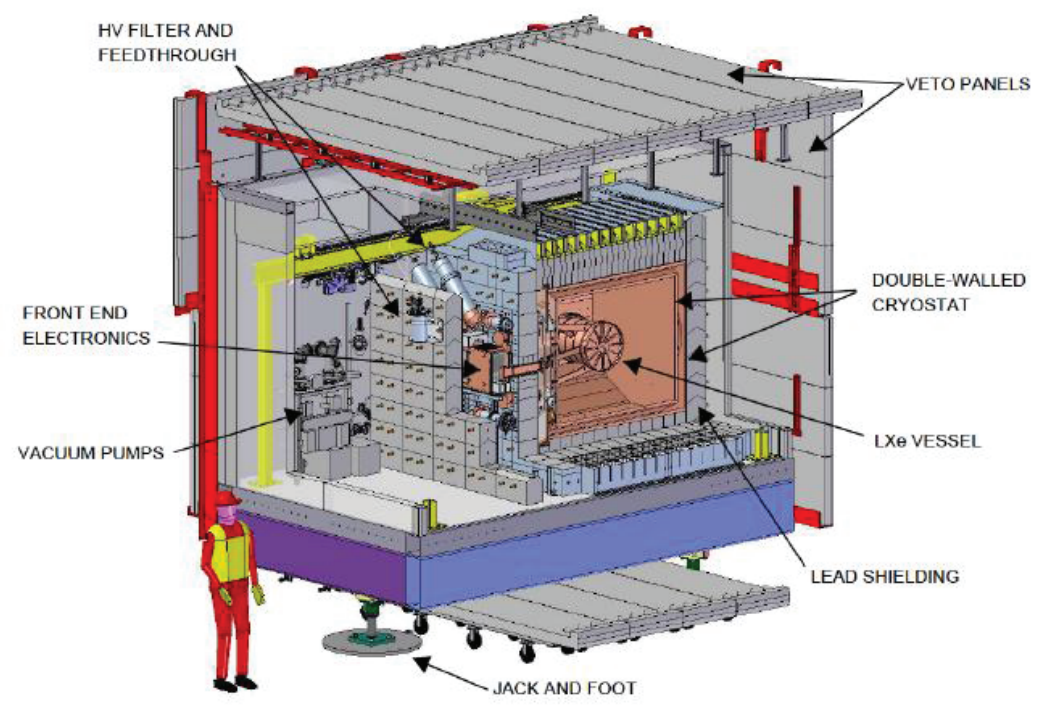

Figure 3.2: A cut-away view of the clean room module at the Waste Isolation Pilot Plant containing the EXO-200 detector. Reprinted from [23].

The TPC vessel is immersed in a bath of HFE-7000 [24] (1methoxyheptafluoropropane) cryofluid which shields the detector from external gamma radiation and regulates the temperature of the TPC. In addition to this, the detector is surrounded by lead shielding and a series of muon veto panels on four sides which detect any cosmic rays passing near the detector. More details on the supporting hardware of the detector can be found in [25].

\section{The Waste Isolation Pilot Plant}

An additional step taken (after HFE/lead shielding and muon veto) to reduce cosmic ray backgrounds in the detector is the choice of location for the experiment. EXO200 is located in the Waste Isolation Pilot Plant (WIPP) near Carlsbad, New Mexico. The WIPP facility is used for the permanent storage of trans-uranic nuclear waste and consists of a surface handling facility on top of a $~ 650 \mathrm{~m}$ deep mine in the Salado salt formation. The EXO-200 TPC is located inside a class 100 clean room inside this mine which provides equivalent shielding of $1585 \mathrm{~m}$ of water. The clean room itself consists of several modules, each containing different components of the system, one of which is pictured in figure 3.2. 


\subsubsection{Event Reconstruction}

When energy is deposited in the TPC (via particles interacting with the LXe) it produces free ionization charge (electrons) and scintillation light (photons at 178 $\mathrm{nm})$. The anode and cathode together produce a uniform electric field which drifts free ionization charges to the vessel end cap where it is detected by the two wire planes (seen as induction in the first plane and charge collection in the second). The scintillation light is detected by the APD clusters at either end of the TPC near instantaneously whereas the free charges take some time to drift depending on the electric field (for Phase-I the drift velocity is $\sim 1.71 \mathrm{~mm} / \mu \mathrm{s}$ ). Because the cathode is $90 \%$ optically transparent, the scintillation signal is detected in both TPCs, whereas charge signal is only detected in the TPC where the event originally occured.

It is now clear how the event position can be determined. Because of the $60^{\circ}$ angle between the shielding wires and collection wires we can use them to define a pair of correlated spatial coordinates, $\mathrm{V}$ and $\mathrm{U}$ respectively which can be easily transformed into the classical orthogonal $\mathrm{X}$ and $\mathrm{Y}$ coordinates (I will hereby refer to the collection wires as $\mathrm{U}$-wires and the shielding wires as $\mathrm{V}$-wires). The orientation of these two coordinate systems is shown in figure 3.3. The $\mathrm{Z}$ coordinate of the event can then be determined by calculating the time difference between the arrival of the scintillation signal and the charge collection signal and multiplying this time difference by the drift velocity in the TPC. In addition to the event position, there are several other useful quantities which can be measured when an event occurs in the TPC.

\section{Multiplicity and the Single-Site/Multi-Site Discriminator}

In some events, a particle passing through the TPC can deposit energy in multiple locations, resulting in signals appearing from multiple locations. A new variable is introduced called the multiplicity which represents the number of resolvable charge clusters observed in a single event. A single charge cluster is considered to be up to two neighbouring U-wires with ionization signals occuring within $3.5 \mu$ s of each other. Events can now be classified as either Single-Site or Multi-Site (SS/MS, multiplicity $=1$ or $>1$ ) categories. This separation is useful because almost all signal-like events $(2 \nu \beta \beta$ and $0 \nu \beta \beta)$ are expected to be in the SS category, therefore by examining the multi-site events additional constraints can be placed on the background model. 


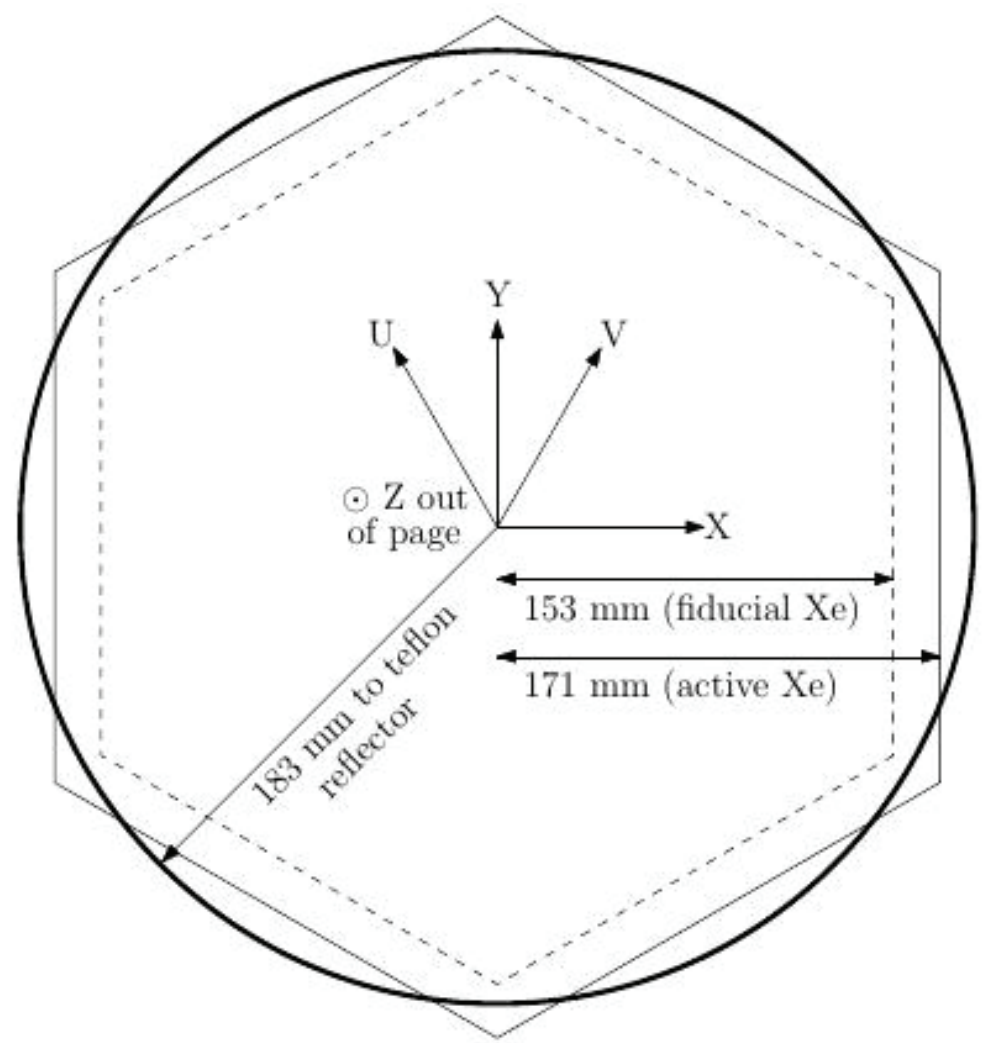

Figure 3.3: Diagram showing the orientation of the U-V and X-Y coordinate systems as well as the fiducial and active regions of the Xe TPC. Reprinted from [23]. 


\section{Standoff Distance}

Using the X-Y-Z coordinates of an event, an additional position related variable can be calculated, called the standoff distance. The standoff distance represents the closest distance from the event to the TPC vessel surface. In the case of a MS events, this is calculated for each cluster and the lowest value is used. This is another useful quantity because signal-like and background-like events are expected to have different spatial distributions, signal-like events should be uniformly distributed within the LXe whereas background-like events should mostly originate from the surfaces of the passive TPC components.

\section{Rotated Energy}

Another important quantity that can be gathered from an event is the event energy. For any given TPC event, the total energy can be calculated from both the ionization signals (seen on U-wires) and scintillation signals (seen on APD clusters). In either case, the energy is calculated by integrating the signal seen over all channels. The response of the detector is calibrated using several radioactive sources with known decay energies (sources summarized in table 3.1). In order to evaluate the spatial dependence of this response, several source positions are used, as illustrated in figure 3.4. The ${ }^{228} \mathrm{Th}$ source is used to calibrate the detector at source position S5 every few days but the rest of the sources and positions are only used periodically during calibration campaigns. These calibration campaigns take place over the course of several days approximately every three months, during which time data is collected for all calibration sources placed at each source position. Calibration data is collected for $\sim 3$ hours for each source/position (dependent on the activity of the source).

Once the ionization energy, $E_{\mathrm{I}}$, and the scintillation energy, $E_{\mathrm{S}}$ have been calculated they can be combined in a way which takes advantage of their anti-correlated nature to minimize the energy resolution at $2615 \mathrm{keV}$ (the $\gamma$ energy of ${ }^{228} \mathrm{Th}$ ). This is done by calculating what is called the rotated energy, $E_{\mathrm{R}}$ defined as,

$$
E_{\mathrm{R}}=E_{\mathrm{S}} \sin \theta+E_{\mathrm{I}} \sin \theta
$$

where $\theta$ is the rotation angle. Single-site and multi-site events use separate rotation 


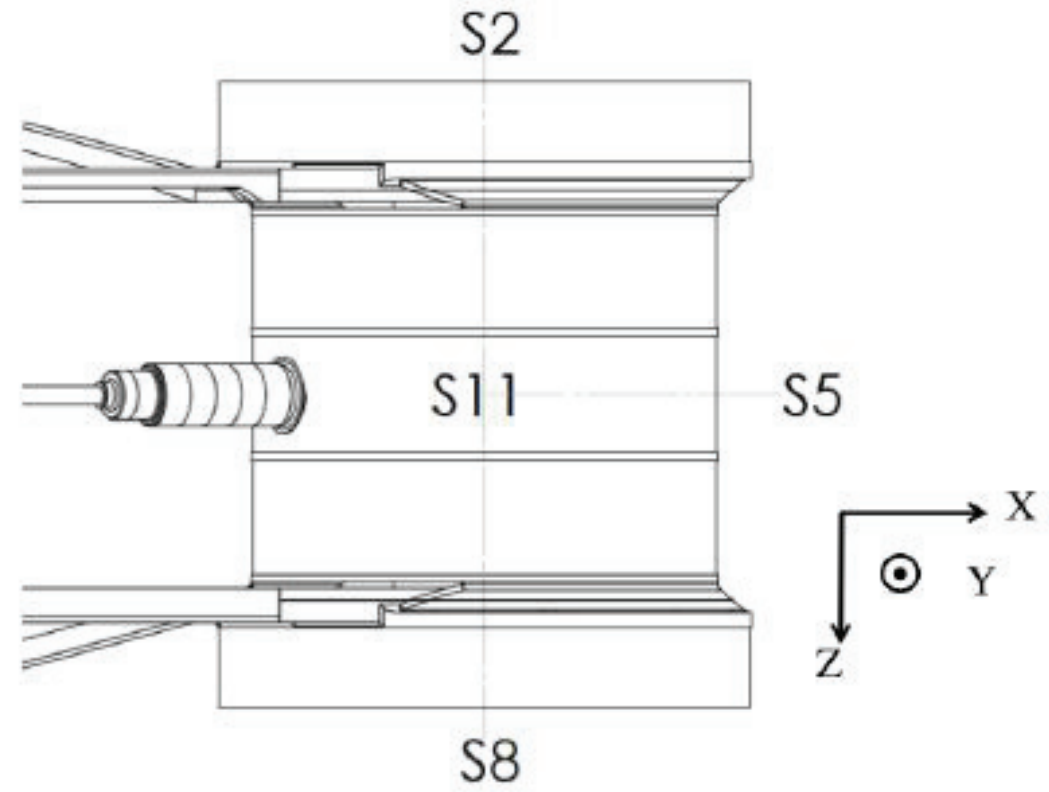

Figure 3.4: Diagram of the calibration source locations in the EXO-200 TPC. The $\mathrm{X}-\mathrm{Y}-\mathrm{Z}$ coordinates of each are as follows: $\mathrm{S} 2=(0,0,-29.5 \mathrm{~cm}), \mathrm{S} 5=(25.5 \mathrm{~cm}$, $0,0), \mathrm{S} 8=(0,0,29.5 \mathrm{~cm}), \mathrm{S} 11=(0,25.5 \mathrm{~cm}, 0) . \mathrm{In}$ addition, $\mathrm{S} 17=(0,-25.5$ $\mathrm{cm}, 0)$ is not shown. Reprinted from [23].

Table 3.1: Basic information on the radioactive sources used for detector calibration in EXO-200.

\begin{tabular}{|c|c|c|}
\hline Source & Activity (Bq) & Half-life (years) \\
\hline \hline${ }^{60} \mathrm{Co}$ & $530 \pm 6$ & 5.27 \\
${ }^{137} \mathrm{Cs}$ & $2820 \pm 33$ & 30.1 \\
${ }^{228} \mathrm{Th}$ & $1417 \pm 17$ & 1.91 \\
${ }^{226} \mathrm{Ra}$ & $255 \pm ?$ & 1600 \\
\hline
\end{tabular}

*Values from [23]. 


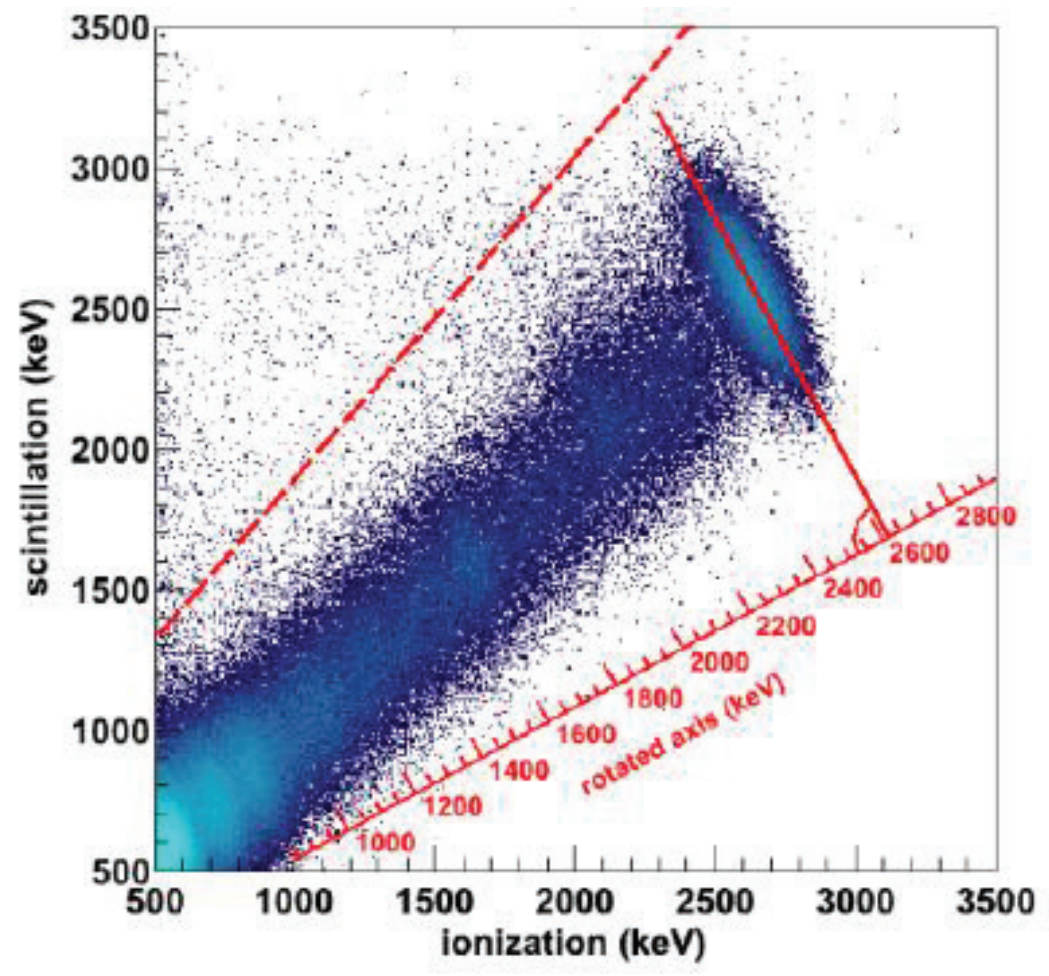

Figure 3.5: Scintillation energy vs. ionization energy for the ${ }^{228} \mathrm{Th}$ source at S5. The minimization of energy resolution by rotation is illustrated here. Reprinted from [19]. 
Table 3.2: EXO-200 effective energy resolution fit results.

\begin{tabular}{|c|c|}
\hline Parameter & Value \\
\hline \hline$\sigma_{\text {elec }}$ & $20.8 \mathrm{keV}$ \\
b & $0.628 \mathrm{keV}^{1 / 2}$ \\
$\mathrm{c}$ & $1.10 \times 10^{-3}$ \\
\hline \multicolumn{2}{|c|}{ Values from [16]. }
\end{tabular}

angles. This rotation is illustrated in figure 3.5.

Since the resolution is dominated by electronic noise in the scintillation readout and is seen to vary with time, a few additional considerations are made to obtain the best possible resolution. To account for the time variation, the optimal rotation angle is recalculated on a weekly basis for both SS and MS. During post-processing, a denoising algorithm is applied to the scintillation signals. The algorithm considers the noise in each APD channel as well as the position of charge depositions in the detector in order to select the best combination of APD waveforms, thus improving energy resolution and further reducing the time dependence.

The rotated energy calibration data is fit to an effective, time-independent energy resolution function [25], $\sigma^{2}(E)=\sigma_{\text {elec }}^{2}+b^{2} E+c^{2} E^{2}$ (where $\sigma_{\text {elec }}$ is the electronic noise contribution) which is used to smear the simulated energy distributions. The fit results ar shown in table 3.2. This results in an effective energy resolution of $1.53 \pm 0.06 \%$ for SS events and $1.65 \pm 0.05 \%$ for MS at the $0 \nu \beta \beta$ Q-value.

\section{$3.2 \quad n E X O$}

The nEXO experiment is another liquid xenon TPC intended to search for neutrinoless double beta decay. Designed as a successor to EXO-200, nEXO is a 5-tonne version of the current $200 \mathrm{~kg}$ detector. With 5-10 years livetime, the nEXO collaboration expects to be able to either discover a neutrino mass in the inverted hierarchy or completely rule it out. 


\subsubsection{The nEXO Experiment}

The nEXO detector is intentionally designed to be as similar to the EXO-200 detector as possible and therefore the majority of the previous information still applies. The primary difference between the two is the greater mass of xenon ( 5 tonne vs. $200 \mathrm{~kg}$ ). This increase in mass has a number of benefits.

The first and most obvious benefit is that the number of $0 \nu \beta \beta$ events observed is directly proportional to the amount of xenon under observation. Unfortunately any xenon related backgrounds will also scale with the amount of xenon so an increase in volume does not correlate to a linear increase in sensitivity. On the other hand, the large volume of xenon benefits from "self-shielding" in which the increased massattenuation of $\gamma$-rays leads to a reduction of backgrounds due to external radiation. Furthermore the large volume provides better event containment, increasing the fraction of events which are deposited entirely inside the detector leading to better event reconstruction.

The nEXO detector will be installed at the SNOLAB facility in Sudbury, Ontario. This facility, located $2 \mathrm{~km}$ underground, was originally the home of the SNO detector and now houses a number of major underground physics experiments mostly focusing on the study of neutrinos and dark matter. The norite rock formation above the facility provides a $\sim 6000 \mathrm{~m}$ water equivalent overburden, approximately four times that of WIPP, increasing shielding from cosmic rays.

One final improvement of nEXO over EXO-200 is the introduction of barium tagging to reduce major backgrounds. While not a part of the initial detector design, the introduction of this system is a planned upgrade to further increase the detector sensitivity. The main concept of barium tagging is to identify the ${ }^{136} \mathrm{Ba}^{++}$daughter ion produced from the double beta decay of ${ }^{136} \mathrm{Xe}$. 


\section{Chapter 4}

\section{EXO-200 Analysis}

In order to determine the effective Majorana-neutrino mass, data from the experiments detailed in chapter 3 must be analyzed. This chapter will explain the current analysis technique used by the EXO-200 collaboration and then explore a new improved version of the analysis technique which has been developed in order to incorporate additional event information and improve results.

\subsection{EXO-200 Previous Analysis}

Once the EXO-200 detector has collected a sufficient amount of events, an analysis can be performed to search for the $0 \nu \beta \beta$ half-lifetime and hence the effective Majorana mass of the neutrino. The analysis performed by EXO-200 is a 2D fitting process in which a probability density function $(\mathrm{PDF})$ is fit to data in order to calculate a distribution of energy vs. standoff distance. The PDF used in the fit will be explained in more detail later in this chapter however here it will suffice to say that it contains parameters modeling the different background components as well as parameters for the $0 \nu \beta \beta$ signal. The events are divided into SS/MS events (effectively adding a third fit dimension with two bins) to further constrain the background model and a binned likelihood fit is performed to fit the aforementioned PDF to the experimental data. The most recently published results of this fit are shown in figure 4.1.

During the fitting process, a negative-log-likelihood (NLL) profile over the number of $0 \nu \beta \beta$ events is created by varying the number of $0 \nu$ events and re-fitting all other floating parameters. The minimized NLL value is the plotted vs. the number of $0 \nu$ events in what is called the NLL profile. This profile is then used to calculate the 


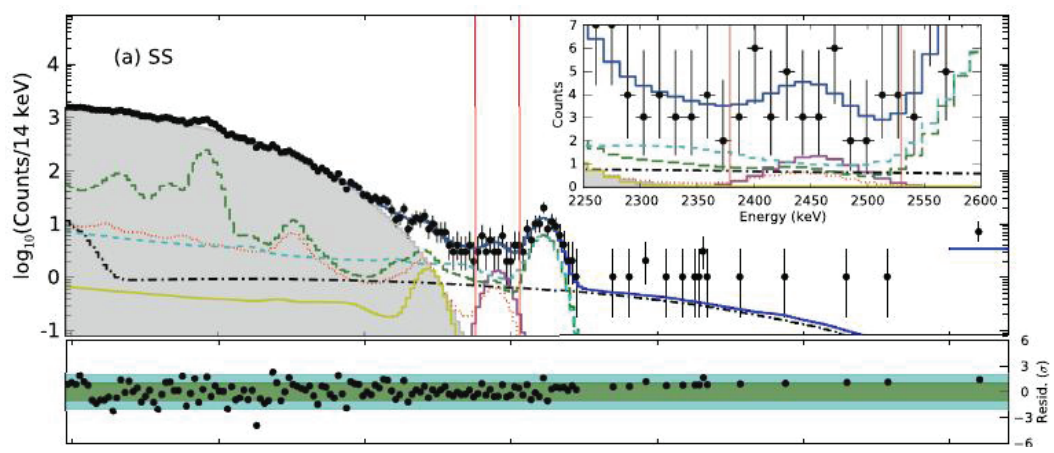

(a)

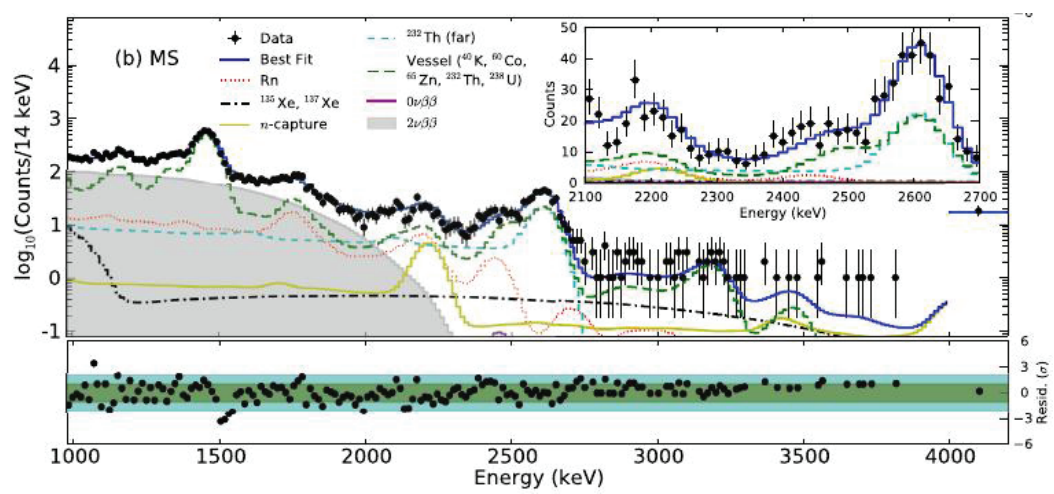

(b)

Figure 4.1: A 1D projection of the fit into energy are shown. Figure 4.1(a) shows the result for SS events and figure 4.1(b) shows the result for MS events. Both reprinted from [16]. 


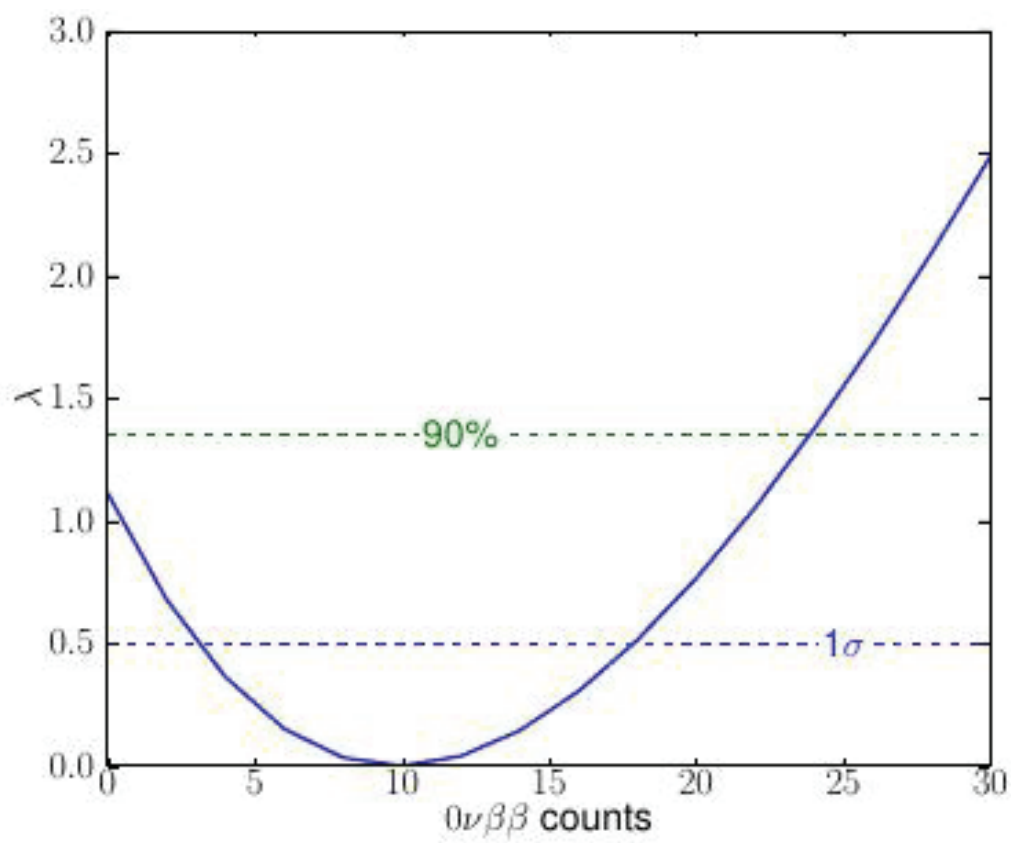

Figure 4.2: The NLL profile for $0 \nu \beta \beta$ counts. The negative-log-likelihood $(\lambda)$ is shown on the y-axis (offset to achieve minimum value of 0 ) vs. the number of $0 \nu$ events on the x-axis. The dashed green (black) line represent the $90 \%(1 \sigma)$ confidence level upper limit on the number of $0 \nu$ events. The minimum near 10 indicates the central value of the fit result. Reprinted from [16].

90\% confidence level upper limit (illustrated in figure 4.2). The upper limit is then used to calculate the half-life upper limit and majorana mass limit. The process of these calculations, along with the process of calculating experimental sensitivity will be discussed in more detail later in this chapter.

\subsubsection{EXO-200 Results}

The most recent results of this analysis, published in Nature in 2014 [16], using the full Phase-I dataset are summarized in table 4.1. At the time of publication this was the best neutrino mass upper limit worldwide.

While the previous EXO-200 analysis used a 2D fit of standoff distance vs. energy with single site/multi site discrimination, it is now possible to include additional information in the analysis that will improve the sensitivity of the experiment. A study was recently published by the EXO-200 collaboration detailing measurements 
Table 4.1: EXO-200 results published in Nature, 2014.

\begin{tabular}{|c|c|}
\hline Property & Value \\
\hline \hline $0 \nu \beta \beta$ half-life sensitivity & $1.9 \cdot 10^{25} \mathrm{yr}$ \\
$0 \nu \beta \beta$ half-life lower limit & $1.1 \cdot 10^{25} \mathrm{yr}$ \\
Majorana neutrino mass upper limit & $190-450 \mathrm{meV}$ \\
\hline
\end{tabular}

*Values from [16].

of the drift velocity and diffusion of electrons within the detector [26]. These new measurements allow significantly better simulation of topological variables such as the number of U-wires with collection/induction signals. These variables provide additional information on event size which can now be used in the search for neutrinoless double beta decay.

When adding information to the analysis, the method of inclusion must be considered carefully. The computation time for a binned likelihood fit is directly proportional to the total number of bins, limiting either the number of fit dimensions or the number of bins in each dimension. Since a reduction in the number of bins would severely limit the sensitivity of the analysis, this places a limit on the number of dimensions in the fit, and so rather than naively adding the new variables as extra fit dimensions, a more sophisticated approach is used. The approach taken is to combine the standoff distance, along with the new variables into a single value using a multivariate analysis (MVA) technique known as a boosted decision tree (BDT) and to use this new variable in place of the standoff distance in the $2 \mathrm{D}$ fit similar to the approach taken in an earlier search for $2 \nu \beta \beta$ decay to excited states [27].

\subsection{Boosted Decision Trees}

Boosted decision trees are a robust MVA technique which have become common in high energy physics (HEP) analysis over the past decade or so (first used by MiniBooNE in 2004 [28]) due to their discriminating power in separating signal from background. At the most basic level, a BDT takes multiple variables describing an event as input and returns a single value, usually ranging from -1 to 1 which indicates the likelihood that the event is either more likely signal (closer to 1) or background (closer to -1). One major application in high energy physics (HEP) was in the search 


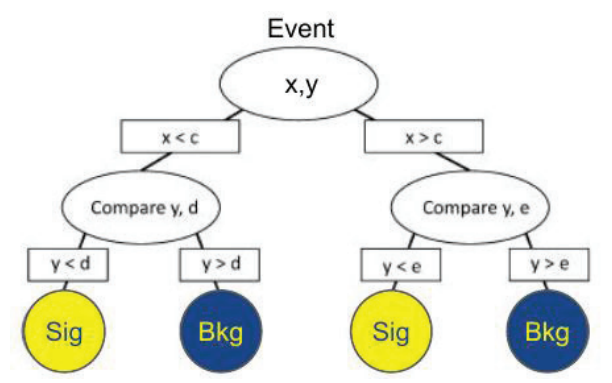

(a)

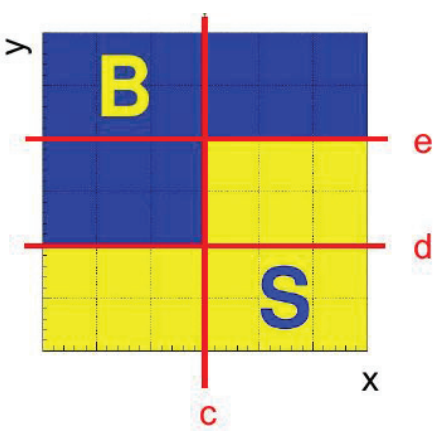

(b)

Figure 4.3: Figure 4.3(a) depicts the decision process used by a simple binary decision tree with two input variables. Figure $4.3(\mathrm{~b})$ shows the regions in 2D parameter space of either signal-like or background-like events defined by the decision tree. Figure 4.3(a) adapted from [31].

for the Higgs boson with the ATLAS detector as detailed in [29] and [30] in which a 2D fit was used, similar to that used by EXO-200, with the BDT variable as one of the fit dimensions.

\subsubsection{BDTs and Binary Decision Trees}

\section{Binary Decision Trees}

One can imagine trying to manually categorize an event described by several variables as either signal or background. The thought process would likely be similar to that shown in figure 4.3(a) in which each variable describing the event is compared to some threshold value and depending on whether the variable is greater or less than this threshold, the event is classified as either signal-like or background-like. This process carves out regions of either signal-like or background-like in the parameter space (the n-dimensional space in which each dimension is one of the variables describing the events) and is known as a binary decision tree. A simple 2D example of this type of parameter space is shown in figure $4.3(\mathrm{~b})$.

\section{Binary Tree Growth}

The process by which a binary decision tree is grown is quite simple, however before an explanation is given, some terminology must be defined. A node of a binary decision 
tree is defined as a point at which the tree splits into multiple branches as the result of a decision (e.g. in figure 4.3(a), there is a node where the variable " $x$ " is compared to the threshold value " $c$ "). An extension to this definition is the root node, which is the first node in the tree. The process of growing a binary decision tree is called training, this term is common to most MVA methods. In order to train a binary tree (or any MVA method), a dataset is required in which it is known which events are signal and which are background. This is referred to as the training dataset.

Now, in order to train a binary decision tree, a simple procedure is followed. First, the signal events and background events in the dataset are normalized such that the weighted sum of signal events is the same as the weighted sum of background events. Beginning at the root node, each input variable is examined in turn. A threshold is chosen for each variable which optimizes the training parameter (typically signal purity or some similar metric) for the dataset and whichever variable provides the greatest increase to the training parameter is used as the decision at the current node, the dataset is then split into subsets based on which events pass or fail the chosen cut and the process is repeated for each new node. This process continues until a termination condition is reached (typically either a minimum dataset size or a maximum tree depth, where the tree depth is the number of nodes passed before reaching a decision). The final nodes of the tree rather than splitting the dataset further, return a classifier score of \pm 1 ( +1 for signal-like, -1 for background-like) based on the purity of the remaining dataset. The purity of the dataset is defined as,

$$
p=\frac{s}{s+b}
$$

where $p$ is the purity, $s$ is the weighted number of signal events and $b$ is the weighted

number of background events. The node is then classified as signal-like if the purity is $>0.5$ (i.e. $>50 \%$ signal) and background-like otherwise. Figure 4.4 shows a flow diagram of the training process.

\section{Boosted Decision Trees}

It is evident that unless signal and background events are perfectly separated from each other (in which case analysis becomes trivial) then a simple binary will likely misclassify a significant portion of both signal and background events. This misclassification is undesirable for a rigorous analysis and is dealt with by combining the 


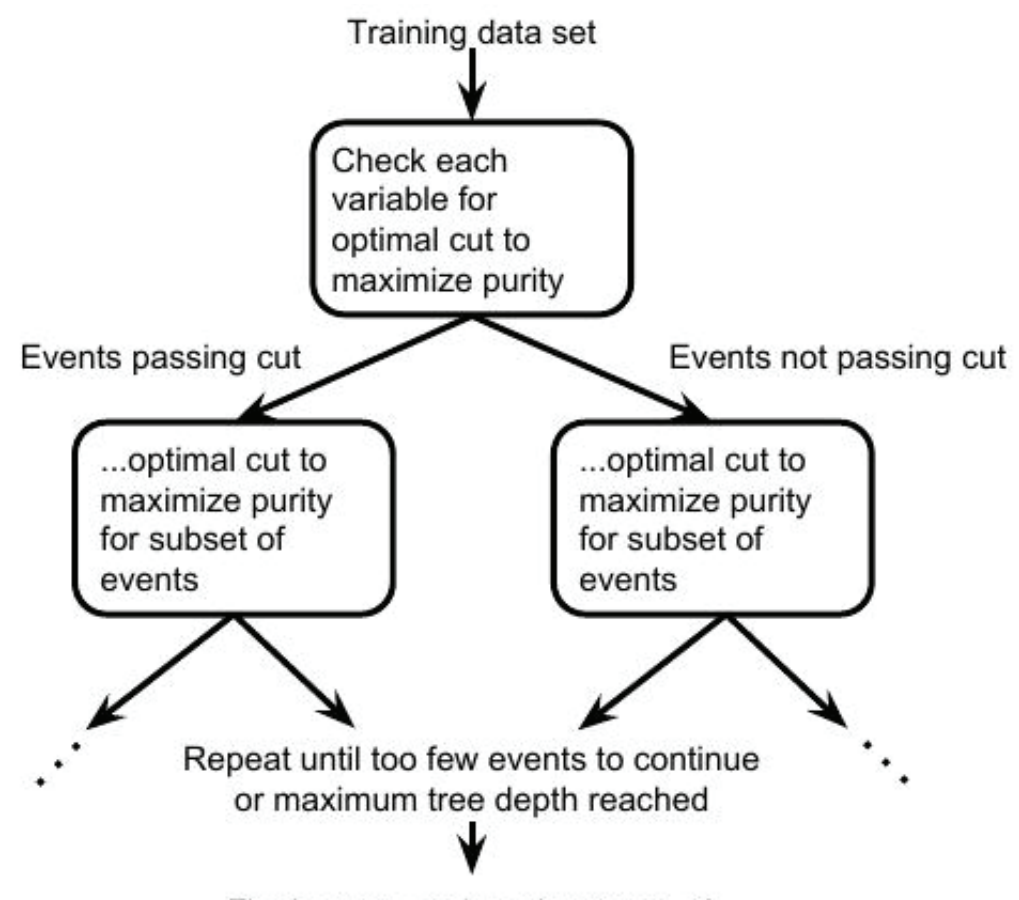

Final cut on each path returns \pm 1

Figure 4.4: The process by which a binary decision tree is trained.

result of multiple binary decision trees into a single value. This is the core structure of a boosted decision tree.

A BDT is composed of a number of binary decision trees, each of which examines the same set of input variable and returns a binary decision $( \pm 1)$. The results of all binary trees are combined via a weighted average into what is called a classifier score, where an individual tree's result is weighted according to the previous tree's misclassification rate (i.e. during training a tree with a high misclassification rate increases the weight of the following tree) as illustrated in figure 4.5(a). This weighted average, rather than defining regions in parameter space of either signal-like or backgroundlike events, now defines many regions of different classifier scores ranging from -1 to 1 (an example of which is shown in figure 4.5(b)). As the number of trees increases, the regions of different scores become a nearly continuous spectrum. The process by which each binary tree in a BDT is created is called boosting, hence the name boosted decision tree. For the analysis described in this thesis, adaptive boosting was used as described in section 4.2.1. 


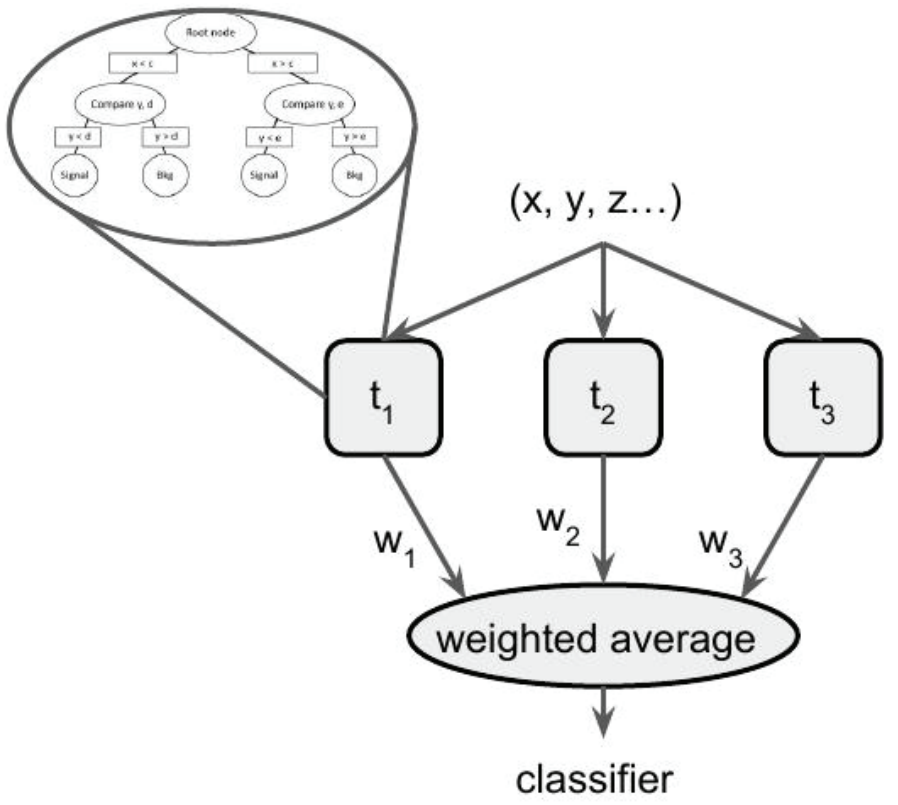

(a)

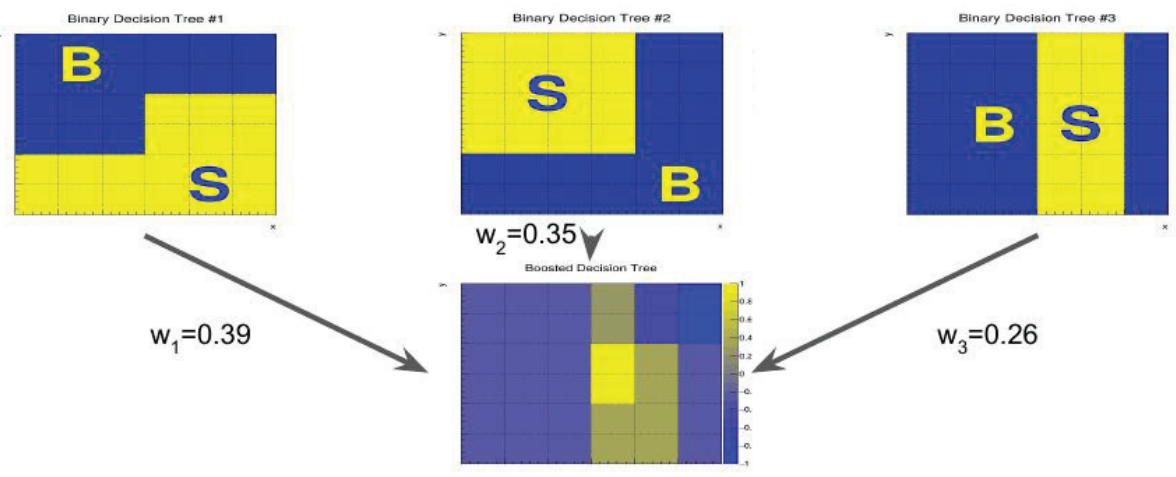

(b)

Figure 4.5: Figure 4.5(a) shows the process by which a BDT calculates its classifier score from the weighted average of binary trees. Here $\mathrm{w}_{1,2,3}$ are the weights of the binary trees, $\mathrm{t}_{1,2,3}$. Figure $4.5(\mathrm{~b})$ depicts the $2 \mathrm{D}$ parameter space of a BDT with three binary trees. There are now regions corresponding to different classifier scores rather than regions of signal or background. Figure 4.5(a) adapted from $[31]$. 


\section{Boosting and Bagging}

It is clear from examining the training process of a binary decision tree that for a given training dataset, training will always result in the same decision tree. This is not useful for the contruction of a boosted decision tree because if every binary tree is identical, then the BDT is no more effective than a single binary tree. In order to create a series of different binary trees the training set must be changed after each tree is built. One method for changing the dataset is to only use a random subset of the full dataset when training each binary tree. This method is called bagging and results in what is called a bagged decision tree (also shortened to BDT). The other method (which is used in this analysis) is to reweight events in the training dataset. The reweighting process is called boosting and results in a boosted decision tree.

To build a BDT, again a training dataset is required and a simple iterative process is followed. The dataset is used to construct a binary decision tree (including the reweighting of signal and background events). After contruction of a binary tree, the misclassification rate of the tree is calculated (using the original, unweighted training dataset) and it is noted which events were classified correctly or incorrectly. The binary decision tree is assigned a weight according to the misclassification rate of the previous tree and the events in the dataset are reweighted (now according to the new tree's misclassification rate). The exact reweighting may vary depending on training settings or boosting method however the basic concept is that events which were misclassified have their weights increased, while events which were properly classified have their weights decreased allowing the total weight of the dataset to stay the same. After this reweighting, the signal and background events are again reweighted such that the sum weights are equal). The reweighted dataset can then be used to train a new binary tree and this process is repeated until the desired number of trees is reached. This process is illustrated in figure 4.6. It is clear that the earliest trees will have the most importance in the final BDT and that the tree weights will drop off rapidly as later trees focus more and more on borderline events which are difficult to classify correctly.

\subsubsection{BDTs in TMVA}

The ROOT data analysis framework provides a useful toolkit for our analysis, called the Toolkit for MultiVariate Analysis (TMVA) [32], which contains implementations 


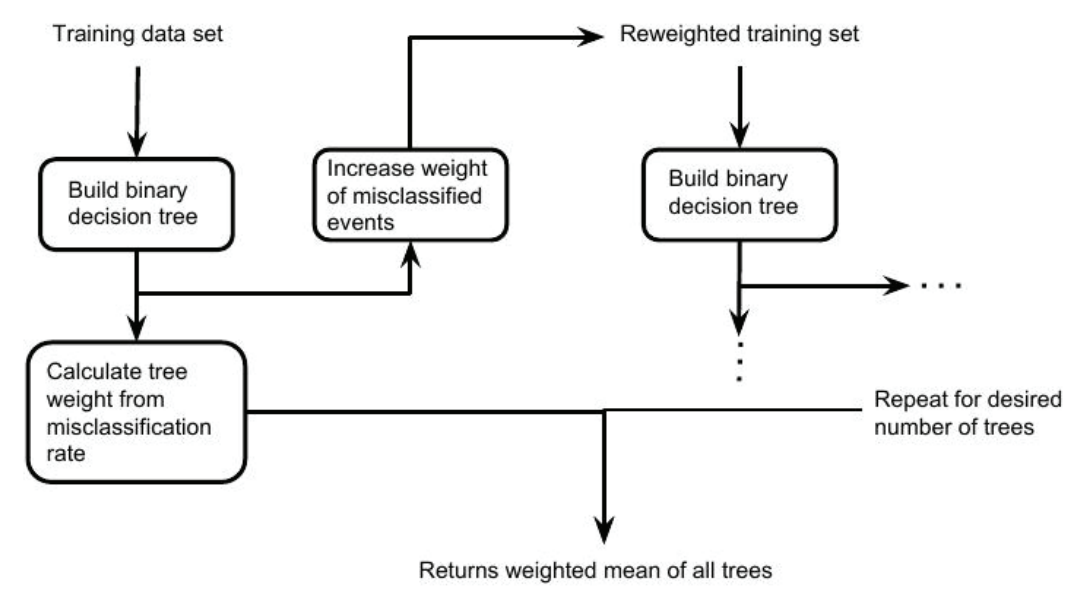

Figure 4.6: The process by which a boosted decision tree is trained.

of a variety of MVA methods including BDTs. The specifics of the chosen BDT method can now be explained within the context of the TMVA settings. Table 4.2 lists some settings used to train the BDT in this analysis. Any settings not listed use the TMVA defaults as described in [32]. The use of nCuts $=-1$ causes TMVA to automatically optimize the value of nCuts, increasing computation time but occasionally resulting in slightly improved BDT performance. This option was tested for use in this study but no significant impact was seen on the results and so the default value of 20 was used. It should be noted that while the BDT in this study is referred to as a Boosted Decision tree, it was trained with bagging enabled, using a bagging fraction of 0.5, making it a hybrid Boosted/Bagged Decision Tree.

\section{Gini Index}

As mentioned in section 4.2.1, when optimizing the thresholds while training the binary decision trees some kind of purity metric is used. The default criteria in TMVA (used in this analysis) is called the Gini Index. This metric is defined as,

$$
g=p \cdot(1-p)=\frac{s b}{(s+b)^{2}}
$$

where $g$ is the Gini index and $p$ is the purity, as defined in equation 4.1 (where $s$ and $b$ are also defined).

During training, in order to calculate the optimal splitting threshold, the Gini index is calculated for each data subset. The threshold is then found which minimizes 
Table 4.2: TMVA options used in training the BDT.

\begin{tabular}{|c|c|c|}
\hline Option & Value Used & Description \\
\hline NTrees & 1000 & Number of trees in the forest \\
\hline MinNodeSize & 0.1 & $\begin{array}{l}\text { Minimum percentage of training } \\
\text { events required in a leaf node (de- } \\
\text { fault: Classification: } 5 \% \text {, Regres- } \\
\text { sion: } 0.2 \% \text { ) }\end{array}$ \\
\hline MaxDepth & 3 & $\begin{array}{l}\text { Max depth of the decision tree al- } \\
\text { lowed }\end{array}$ \\
\hline AdaBoostBeta & 0.5 & $\begin{array}{l}\text { Learning rate for AdaBoost algo- } \\
\text { rithm }\end{array}$ \\
\hline UseBaggedBoost & True & $\begin{array}{l}\text { Use only a random (bagged) sub- } \\
\text { sample of all events for growing } \\
\text { the trees in each iteration. }\end{array}$ \\
\hline BaggedSampleFraction & 0.5 & $\begin{array}{l}\text { Relative size of bagged event sam- } \\
\text { ple to original size of the data } \\
\text { sample (used whenever bagging is } \\
\text { used (i.e. UseBaggedBoost, Bag- } \\
\text { ging,) }\end{array}$ \\
\hline nCuts & 20 & $\begin{array}{l}\text { Number of grid points in variable } \\
\text { range used in finding optimal cut } \\
\text { in node splitting. }\end{array}$ \\
\hline
\end{tabular}

${ }^{*}$ Descriptions from [32]. 
the sum of the two Gini indices.

TMVA offers several other metrics for cut optimization however it was found that the Gini Index provided the best BDT performance.

\section{Adaptive Boosting}

Another setting mentioned in section 4.2.1 was the specific boosting method. In this BDT, TMVA's default method, AdaBoost (short for Adaptive Boost) was used. The adaptive boost method calculates a common boost weight, $\alpha$, for each binary tree as it is built. All misclassified events have their weights multiplied by this $\alpha$ and then the total dataset weight is renormalised such that it remains constant. Here $\alpha$ is given by,

$$
\alpha=\frac{1-e}{e}
$$

where $e$ is the misclassification rate of the tree.

The weight of the tree itself (used in the weighted average) is then $\ln (\alpha)$, such that the final BDT classifier score is given by,

$$
y_{\text {Boost }}(\mathbf{x})=\frac{1}{N_{\text {collection }}} \cdot \sum_{i}^{N_{\text {collection }}} \ln \left(\alpha_{i}^{\beta}\right) \cdot h_{i}(\mathbf{x})
$$

where $y_{\text {Boost }}$ is the BDT response, $h_{i}$ is the response of the $i$-th binary decision tree (i.e. \pm 1$), N_{\text {collection }}$ is the total number of binary trees and $\mathbf{x}$ is the set of all input variables. In this equation, there is an additional parameter, $\beta$, called the adaptive boost learning rate (AdaBoostBeta). This parameter rescales the boost weight, allowing the user to slow the learning of the BDT, spreading the weights more evenly across all trees.

\subsubsection{An Unbiased BDT}

As with any type of analyis, it is important when training a BDT to avoid biasing the results. The two primary types of bias that can be encountered when training a BDT are overtraining (also known as overfitting), where a BDT is affected by statistical fluctuations in the training dataset, and Monte Carlo bias, which is the bias towards simulated data as opposed to real physical data. 


\section{Over Training}

Over training is an effect which can occur in any multivariate analysis technique. It is usually caused by training on a dataset which does not accurately represent the full dataset on which the BDT will be applied (either too few events are used or the selection does not span the full parameter space of the dataset). This potentially allows the BDT to pick up on trends in the training set which do not appear in the full data set. While this form of bias is always present to some degree, some precautions can be taken to minimize it's effects. The simplest solution to this is to ensure that the training set properly represents the full data set. This is typically done by selecting a large number of events and splitting this data set into statistically independent data sets, a training set and a testing set. A Kolmogorov-Smirnov test (K-S test) is used to check that the training and testing sets originate from the same events distribution. The BDT can then be built using the training set and then the response will be checked on both the training and testing sets and compared (again using the K-S test). If the BDT shows the same response to both the training and testing sets then it is said not to be overtrained. The K-S test is a statistical test of equality of two probability distributions, explained in more detail in [33].

\section{Monte Carlo Bias Due to Modelling}

Another type of bias, Monte Carlo (MC) bias, can occur when a BDT (or any other MVA method) is trained using Monte Carlo data rather than real physics data. This effect occurs when the Monte Carlo does not accurately represent the real life data, either due to low statistics or inaccuracies in the simulation. The solutions to these problems are quite obvious, although not necessarily simple, simulate more or simulate better. In order to avoid this bias, checks must be made to ensure that the $\mathrm{MC}$ is a good representation of real physics data. This is most easily done if there is real data available to compare with the MC (for example, in EXO-200 source calibration data is used). A statistical test can then be used to compare the data and $\mathrm{MC}$ for

consistency. The K-S test is an appropriate test, however historically EXO-200 uses the residuals, defined as,

$$
r_{\mathrm{i}}=\frac{O_{\mathrm{i}}-E_{\mathrm{i}}}{E_{\mathrm{i}}}
$$

to evaluate data/MC agreement and so the same metric is used in this analysis. Here 
$r_{\mathrm{i}}$ denotes the residual of bin $\mathrm{i}$, which contains $O_{\mathrm{i}}$ events of data and $E_{\mathrm{i}}$ events of MC. Note that the residuals approach 0 as data/MC agreement improves.

Unfortunately it is not always the case that real physics data is available for comparison, for instance in the case of $0 \nu \beta \beta$. In this case it must suffice to ensure that the simulation is theoretically sound. It also be useful in this case to check that theoretically similar events (e.g. $2 \nu \beta \beta$ ) are well simulated.

\subsection{EXO-200 Monte Carlo Simulation}

The Monte Carlo which is used in the training of the BDT is generated in two stages. The first stage is the simulation of energy deposits in the detector using a GEANT4 [34] based simulation of the detector geometry. The second stage converts the output of the first stage into electronic signals similar in format to those measured by the actual detector. The signals produced by this simulation can then undergo the same reconstruction process as the real data.

The simulation in GEANT4 used custom coded decay schemes to simulate all necessary isotope nuclear decays and particle interactions within the detector geometry. The simulated geometry includes detailed models of the TPC and it's inner components as well as some of it's surroundings including the HFE, cryostat and lead shield. The geometry is coded using GEANT4 shape primitives and therefore some of the more complex components are reduced to approximate geometries (shown in figure 4.7). These approximations can potentially cause differences between data and MC however the differences are expected to be small and checks are made using source calibration data to ensure there are no major problems.

In simulating the waveforms produced by the energy deposits from the first stage, a few considerations are made. When simulating the waveforms on $\mathrm{U}$ - and $\mathrm{V}$-wires, the simulation accounts for ionization loss, which is tied to the LXe purity, and for the diffusion of charge within the detector [26]. The diffusion is simulated using a 3-D map of the electric field. Once the waveforms are simulated, real noise is then added which has been sampled from each wire. The waveforms on the APD plane are treated differently, accounting only for the varying APD response based on the location of the energy deposition. This is done based on a 3-D light map constructed by placing the ${ }^{228} \mathrm{Th}$ calibration source at various positions in the detector and measuring the $\mathrm{APD}$ response [23]. Once the APD waveforms have been constructed, real noise is 


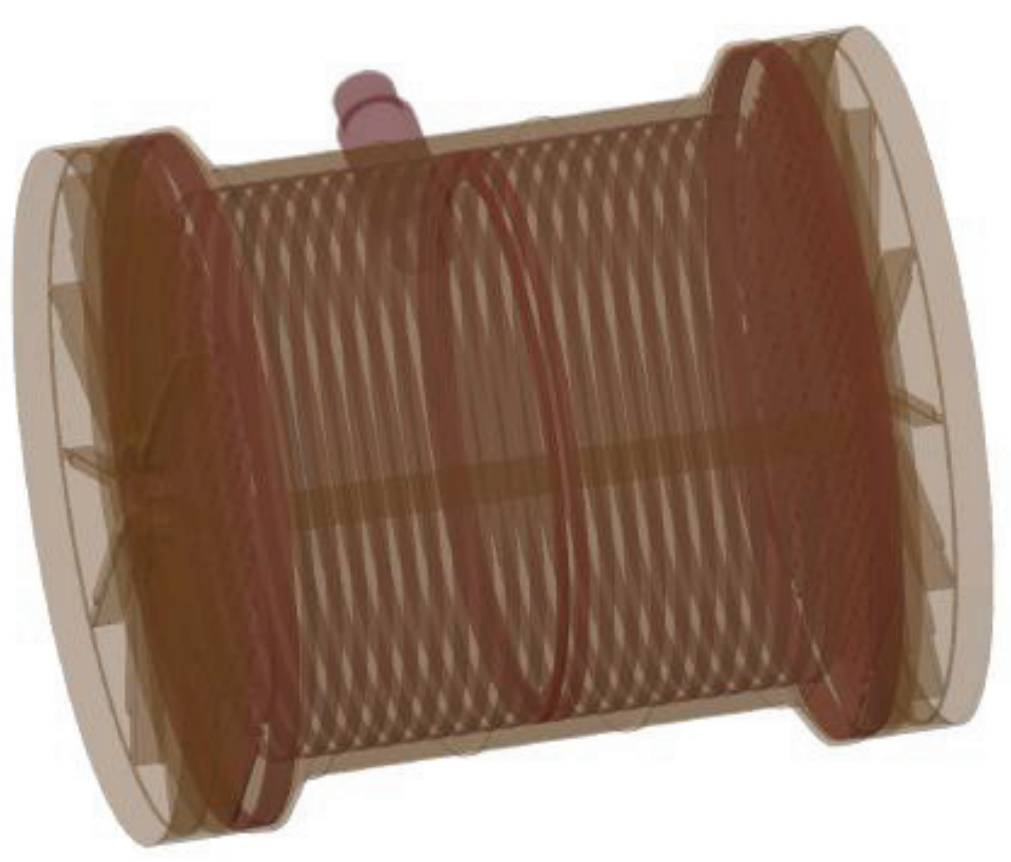

Figure 4.7: Simulated EXO-200 TPC geometry in GEANT4, showing the TPC vessel and and copper internal components. Reprinted from [23].

added sampled from the sum APDs.

Monte Carlo data sets were produced for all non-negligible detector background components including interactions of external $\gamma$-rays with the detector, backgrounds due to radioactive contamination of the detector assembly, radon decay chain backgrounds, cosmogenic backgrounds and muon induced backgrounds (a full list will be shown when discussing the analysis). In addition to backgrounds, Monte Carlo was produced for $0 \nu \beta \beta$ events in the TPC and for each calibration source.

Because the Monte Carlo is vital to the EXO-200 analysis it is important to verify the accuracy of the simulations. The primary method of verification is a direct comparison of Monte Carlo and source calibration data. It is important to note however that there are some major differences between the calibration sources and the $2 \nu \beta \beta$ events which constitute the largest detector background. The $2 \nu \beta \beta$ signal is $\sim 95 \%$ single-site whereas the calibration sources are roughly $\sim 20-50 \%$ single-site. Furthermore, calibration source events are highly localized near the source positions as opposed to $2 \nu \beta \beta$ which are uniformly distributed in the LXe. The final and greatest difference is that the $2 \nu \beta \beta$ are beta-particles, i.e. electrons, whereas the calibration sources are all $\gamma$-rays, thus they undergo different types of interactions within 
the TPC. Because of these differences it is useful to augment the source calibration information with direct analysis of low-background physics data [23].

\section{Evaluation of Systematic Errors Using Monte Carlo}

Unfortunately any modelling involved in the generation of Monte Carlo introduces a degree of systematic error. In order to evaluate the systematic errors in EXO-200, data collected using calibration sources (see Table 3.1) is compared to Monte Carlo simulation of each source. The residuals are evaluated (as in equation 4.5) and the absolute value of the residuals are fit to a linear function. This process is repeated for all required variables and the resulting functions are used to calculate the systematic errors for corresponding values. How these errors are propagated to the final result is explored further in section 4.5.3.

\subsection{A $\beta$-like $/ \gamma$-like BDT Discriminator}

The BDT chosen for this analysis was designed to separate $\beta$-like events $(0 \nu$ and $2 \nu$ ) from $\gamma$-like events (most of our backgrounds). It was trained using $0 \nu \beta \beta$ Monte Carlo as signal and a mix of all $\gamma$-like background components as background with the number of events used for each component based on the background model from a previous analysis.

In order to select the variables that would be used in construction the BDT, two criteria were examined. The first criterion was the Data/Monte Carlo agreement which was evaluated by looking at the residuals as defined in equation 4.5. The residuals were calculated for single-site events using data and Monte Carlo from the ${ }^{228}$ Th calibration source (background-like events) and $2 \nu \beta \beta$ (signal-like). The $2 \nu \beta \beta$ data was obtained by selecting low-background physics data from 980 to $2000 \mathrm{keV}$ and cutting between 1400 and $1500 \mathrm{keV}$ to remove the ${ }^{40} \mathrm{~K}$ peak. This range of energy is expected to be $>90 \% 2 \nu \beta \beta$ events. When examining the residuals, values from 0.1 to 0.1 (i.e. agreement within $10 \%$, hence systematic error $<10 \%$ ) were generally considered to be acceptable for use in the BDT with the effects of any disagreement being propagated forward as systematic error (as described in section 4.5.3), however some leniency was allowed in the case of bins containing only a few events since these are not expected to significantly impact the analysis. The second criterion was the separation [32], defined as, 


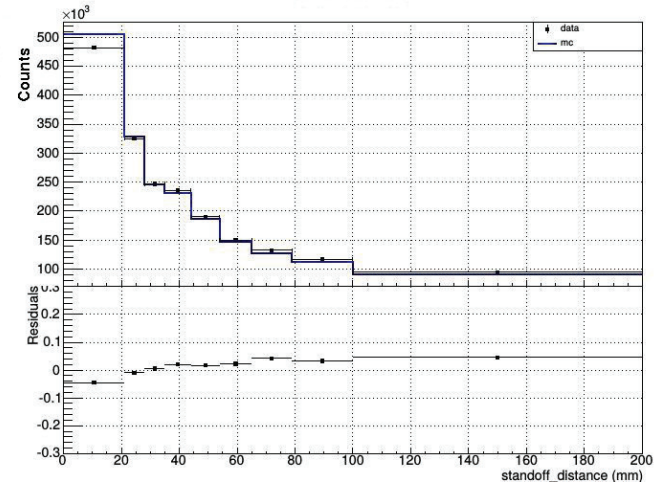

(a) ${ }^{228} \mathrm{Th}$ shape agreement.

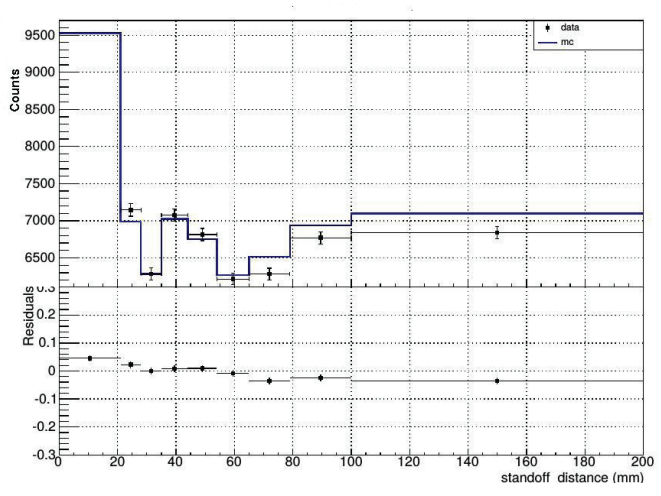

(b) $2 \nu \beta \beta$ shape agreement.

Figure 4.8: Standoff distance shape agreement showing event distributions (upper) and residuals (lower) for both ${ }^{228} \mathrm{Th}$ and $2 \nu \beta \beta$ events. Here a non-uniform binning is used in which we expect signal-like events to be near-uniformly distributed. Statistical uncertainties are shown, systematic uncertainties are omitted.

$$
\left\langle S^{2}\right\rangle=\frac{1}{2} \int \frac{\left(\hat{y}_{\mathrm{S}}(y)-\hat{y}_{\mathrm{B}}(y)\right)^{2}}{\hat{y}_{\mathrm{S}}(y)+\hat{y}_{\mathrm{B}}(y)} d y
$$

where $\left\langle S^{2}\right\rangle$ is the separation, $\hat{y}_{\mathrm{S}}$ is the signal PDF and $\hat{y}_{\mathrm{B}}$ is the background PDF. The separation can take values ranging from 0 to 1 , where a value of 1 represents perfect separation of signal and background and 0 represents identical distributions of signal and background. No particular cutoff separation was required for including a variable in the BDT however preference was given to those which showed the greatest separation between signal and background events. The separation was also evaluated between $0 \nu$ and $2 \nu$ double beta decay events as a check that all beta-like events behave similarly. Like the residuals, the separation was evaluated only for single-site events, since most of the $0 \nu \beta \beta$ signal is expected to be single-site.

\section{Standoff Distance}

The first variable considered for use in the BDT was the standoff distance. Since this variable was used in previous analyses it was an obvious choice for inclusion. The Data/MC agreement is shown in figure 4.8 where $|\mathrm{r}|<0.1$ for both ${ }^{228} \mathrm{Th}$ and $2 \nu \beta \beta$ events. The distributions of background-like, $0 \nu$ and $2 \nu$ events are shown in figure 


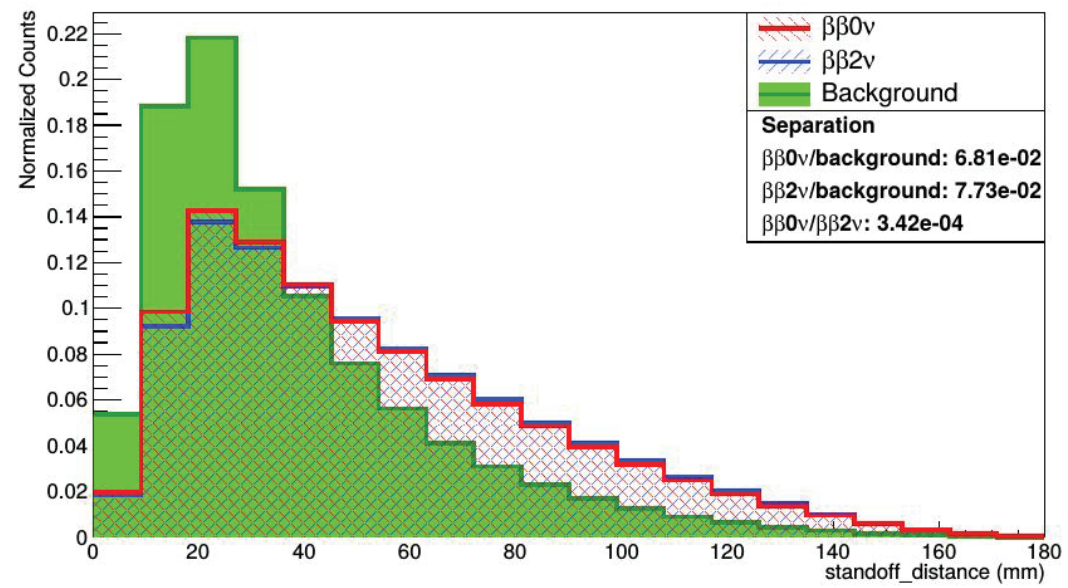

Figure 4.9: Standoff distance distributions for $0 \nu \beta \beta, 2 \nu \beta \beta$ and background events with calculated separation scores between each.

4.9 where a separation of $\sim 0.07$ was calculated between $0 \nu \beta \beta$ and background.

\section{Cluster Risetime}

The second variable chosen for use in the BDT was the cluster risetime. This the time difference between the $5 \%$ and $95 \%$ pulse peak amplitude threshold of a U-wire waveform (summed in the case of more than one wire in a cluster). To understand exactly what this means, we must examine in more detail how signals are identified on waveform traces. Each waveform is first subjected to a matched filter, a threshold is calculated and amplitudes exceeding the treshold are identified as pulses (see figure 4.10 for an example of a U-wire pulse before and after the matched filter). When a pulse has been identified, the waveform is subjected to an unshaping algorithm which calculates the amount of charge deposited, the waveform is then reshaped, subjected to a moving average filter and another peak search is performed to look for additional pulses. Once all pulses in a waveform have been located, a $\chi^{2}$ fit is performed using MIGRAD [35], fitting the pulses to their corresponding signal model (U-wire, V-wire or APD sum signal) in order to calculate the amplitude and timing of each pulse. Figure 4.11 shows a pair of pulses which have been fit to their corresponding signal models.

A few extra characteristics are also calculated directly from the waveforms in order to distinguish between charge collection and induction signals. This distinction 

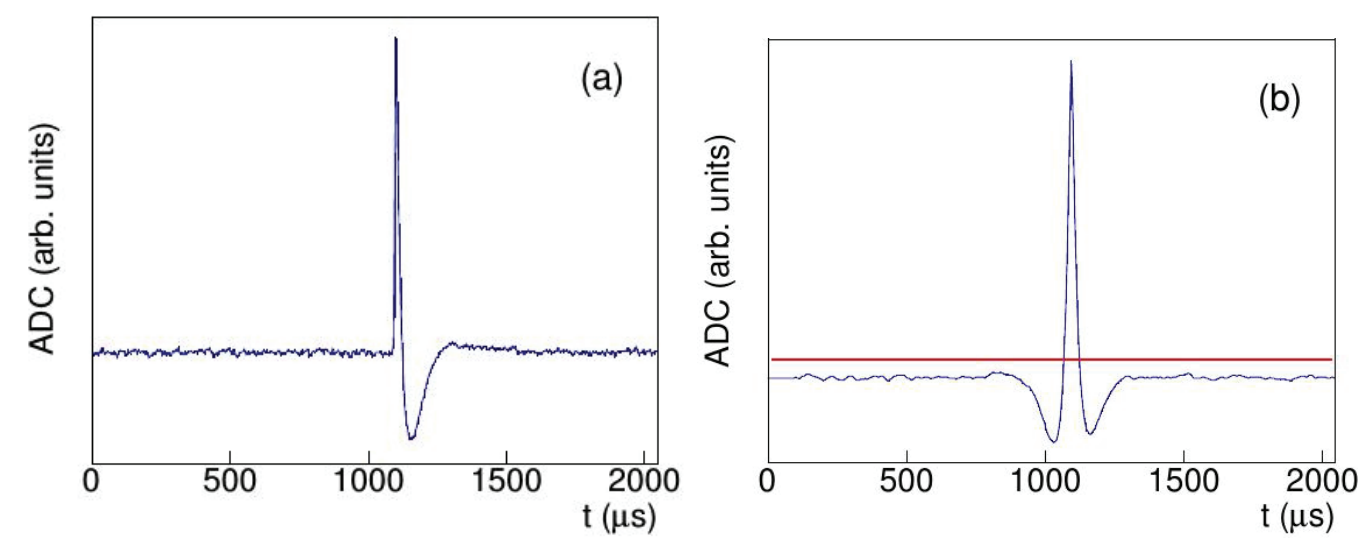

Figure 4.10: Figure 4.10(a) shows a U-wire waveform and figure 4.10(b) shows the matched filter result and corresponding pulse threshold. Both reprinted from [23].

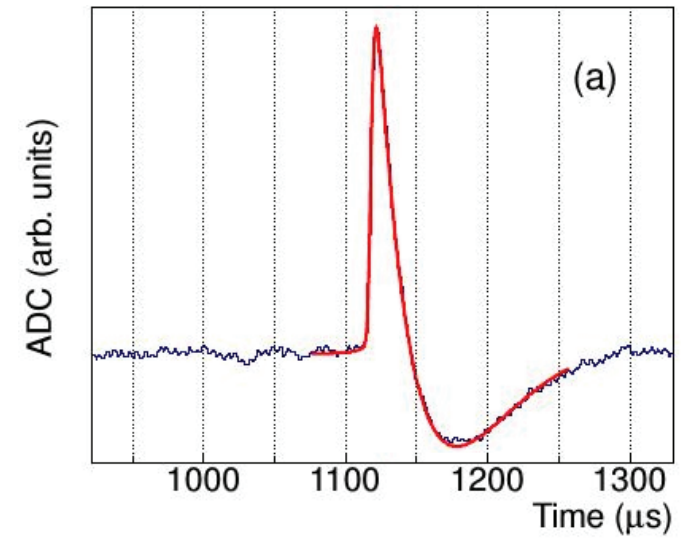

(a) U-wire signal

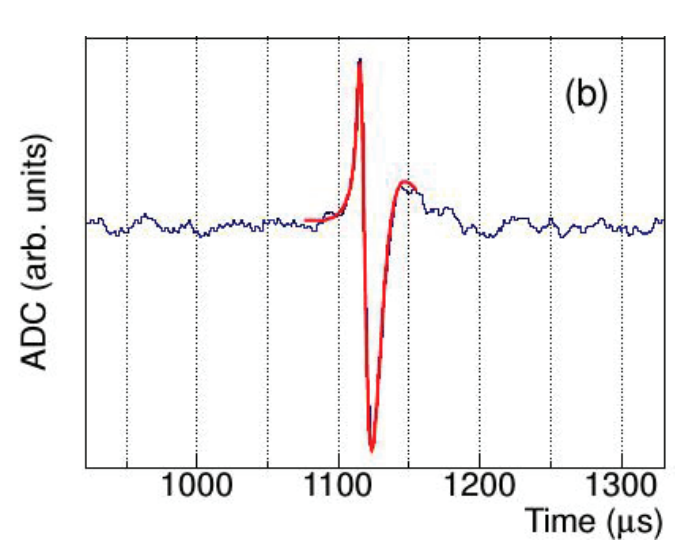

(b) V-wire signal

Figure 4.11: U- and V-wire waveforms fit to their corresponding signal models. Both reprinted from [23]. 
is needed in order to avoid misclassifying single-site events as multi-site (for instance if an induction signal were identified as a small amplitude charge collection signal). Among the characteristics calculated here, the rise time is of primary interest. It is defined as the time measured from when a pulse last crosses a minimum threshold ( $5 \%$ of the pulse height above baseline) to when the pulse first crosses a maximum threshold ( $95 \%$ of the pulse height above baseline).

The cluster risetime is of interest because it relates to how spread out an event is across the Z-coordinate of the TPC. In the case of multi-site events, which contain more than one charge cluster, the cluster risetime is actually a vector quantity which is unsuitable for use in the BDT. To avoid this, the longest cluster risetime is selected (called cluster risetime max) and this is the variable which is used in the BDT. Figure 4.12 shows the data/MC shape agreement for the cluster risetime max. The cluster risetime is found to have considerable residuals $(|r|>0.2)$ at both the lower and upper ends of the range. To compensate for this large disagreement, a range is chosen (indicated by the red vertical bars in figure 4.12) within which the residuals are considered acceptable (6600 - 7900 ns). For the purposes of training the BDT, events outside of this range have their risetimes set to the nearest threshold (i.e. if risetime < threshold $_{\min }$ then risetime $=$ threshold $_{\min }$ and similarly for the upper threshold). This process, called profiling, prevents the BDT from making any decisions outside of the selected range therefore avoiding the propagation of the disagreement into the discriminator variable.

Figure 4.13 shows the risetime distributions of $0 \nu, 2 \nu$ and background events. A separation of $\sim 0.08$ is calculated between $0 \nu \beta \beta$ and background events, a value slightly better than that of the standoff distance which was used in previous analyses. An interesting feature of the risetime distributions is that the $0 \nu$ and $2 \nu$ events actually have a non-negligible separation $(\sim 0.02)$. This separation has been studied and was found to be an effect of energy (higher energy $2 \nu$ events follow a distribution closer to that of the $0 \nu$ events). Despite the somewhat poor data/MC agreement (residuals as high as $\sim 0.2$ even in "acceptable" range), the separation power provided by the risetime makes it a strong candidate for a BDT input variable, therefore the requirement of $|r|<0.1$ is relaxed to $|r|<0.2$. 


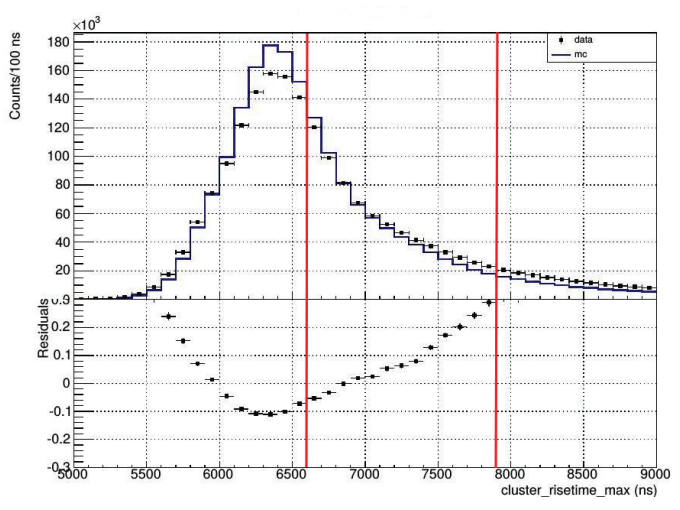

(a) ${ }^{228} \mathrm{Th}$ shape agreement.

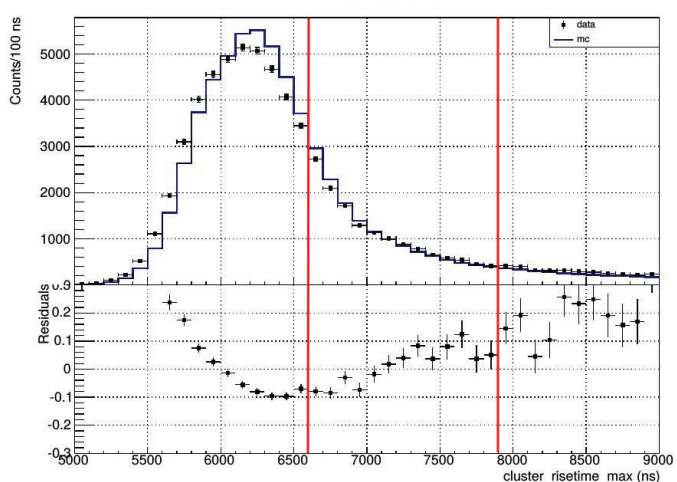

(b) $2 \nu \beta \beta$ shape agreement.

Figure 4.12: Cluster risetime shape agreement showing event distributions (upper) and residuals (lower) for both ${ }^{228}$ Th and $2 \nu \beta \beta$ events. Red vertical lines indicate the upper and lower risetime profiling thresholds. Statistical uncertainties are shown, systematic uncertainties are omitted.

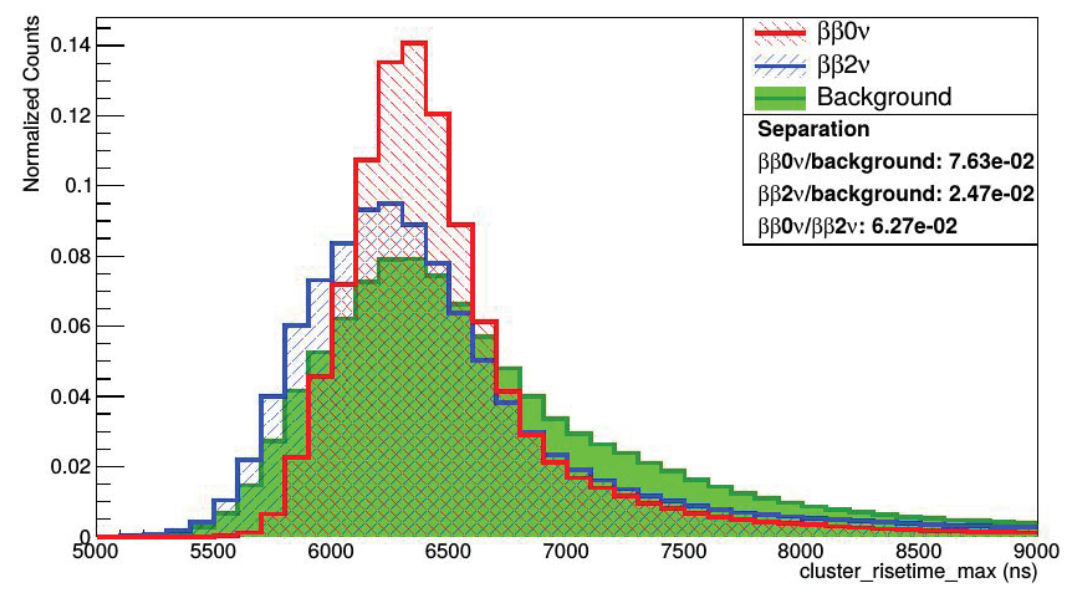

Figure 4.13: Cluster risetime distributions for $0 \nu \beta \beta, 2 \nu \beta \beta$ and background events with calculated separation scores between each. 


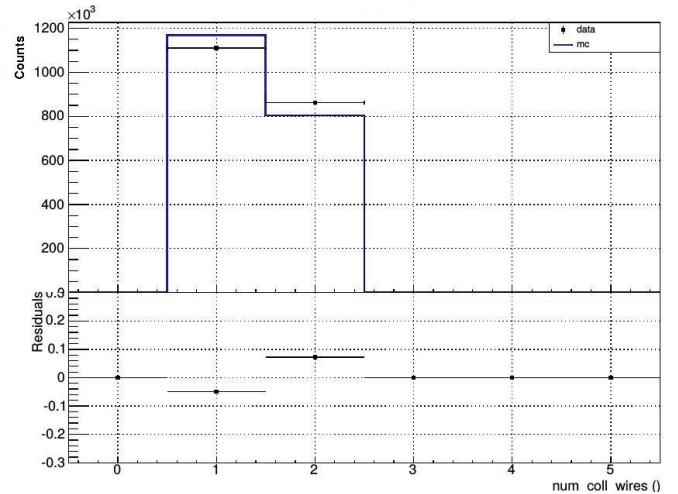

(a) ${ }^{228} \mathrm{Th}$ shape agreement.

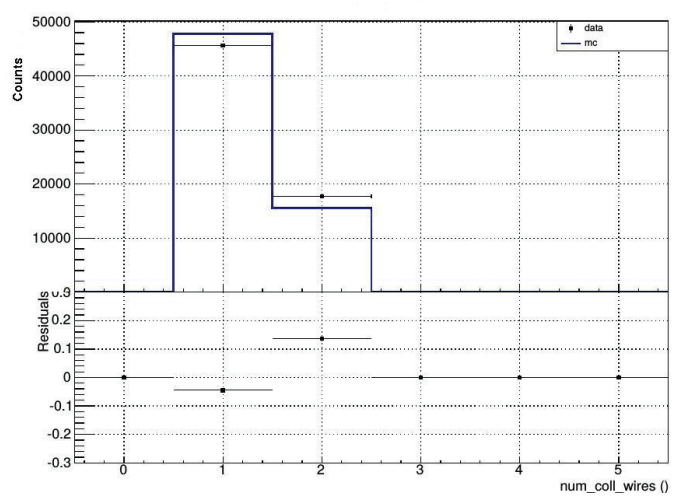

(b) $2 \nu \beta \beta$ shape agreement.

Figure 4.14: Collection wire shape agreement showing event distributions (upper) and residuals (lower) for both ${ }^{228} \mathrm{Th}$ and $2 \nu \beta \beta$ events. Statistical uncertainties are shown, systematic uncertainties are omitted.

\section{Number of Collection Wires}

The final variable chosen for use in the BDT was the number U-wires with charge collection signals (referred to as collection wires for brevity). As the name implies this is the number of U-wires in which a charge collection signal is seen in an event. This variable relates to how spread out an event is in the X-Y plane of the TPC since a more spread out event will be detected on more wires. Due to the discrete nature of this variable it always takes on integer values and in the case of single-site events, only the values 1 and 2. The data/MC agreement for the number of collection wires can be seen in figure 4.14 where it was seen that for the most part $|r|<0.1$. The bin containing events with two wires for $2 \nu \beta \beta$ events showed a slightly larger disagreement $(\sim 0.15)$ however this was attributed to background contamination of the $2 \nu$ data $(2 \nu \beta \beta$ data contains $\sim 10 \%$ background contamination). Figure 4.15 shows the collection wire distributions for $0 \nu, 2 \nu$ and background events with a calculated separation between the $0 \nu$ and background distributions of $\sim 0.02$.

\section{Rejected Variables}

A series of other variables were initially considered for use in the BDT as well however most were quickly dismissed due to poor shape agreement and weak separation power. Chief among these variables was the number U-wires with induction signals (similar 


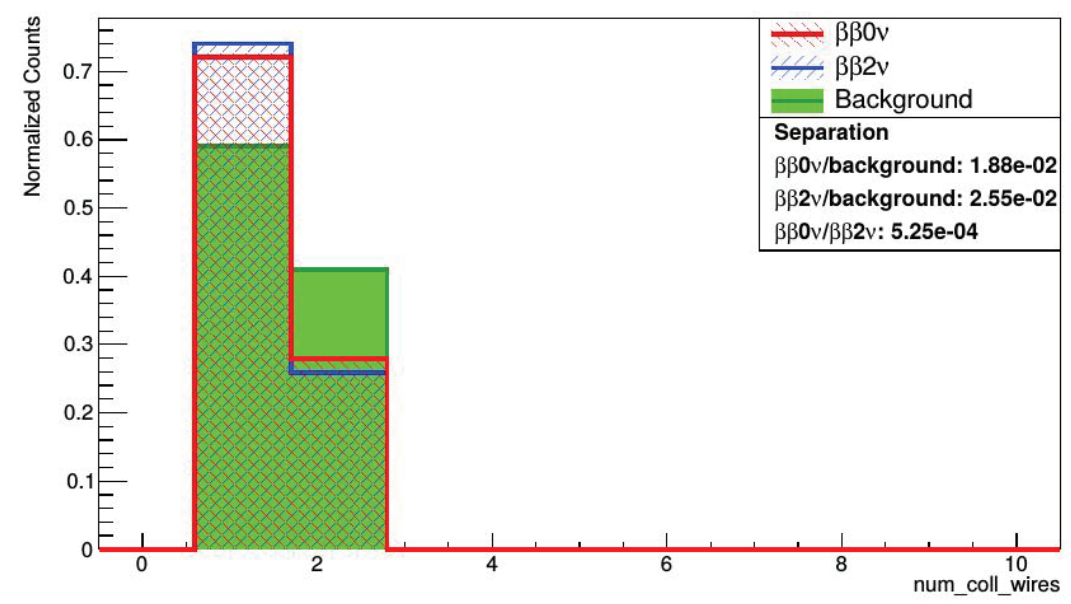

Figure 4.15: Collection wire distributions for $0 \nu \beta \beta, 2 \nu \beta \beta$ and background events with calculated separation scores between each.

to the number of collection wires except counting induction signals instead of charge collection). The number of induction wires was initially found to have poor data/MC agreement however corrections to electron drift velocity in the Monte Carlo simulations brought about some improvement in both induction wire shape agreement and cluster risetime. The induction wires however still provided very little separation and were eventually dropped from the analysis.

In addition to the number of induction wires, several minimal spanning tree (MST) metrics were considered. These metrics were originally intended for use in the analysis in a similar capacity to how the BDT discriminator is now used (i.e. combining multiple topological variables into a single value) except much simpler in form. These variables were found to have very poor data/MC agreement and provided little additional separation and were therefore discarded.

\section{The BDT Discriminator}

One final check that must be made after choosing the input variables is to verify that the correlations between them is well simulated. The strength of boosted decision trees comes from their ability to carve our regions of signal in a phase space of multiple highly correlated variables, therefore it is important the variables used are correlated similarly in both real physics data and the Monte Carlo used to train the BDT. To check that the correlations are consistent, correlation matrices were calculated for data and $\mathrm{MC}$ for both ${ }^{228} \mathrm{Th}$ and $2 \nu \beta \beta$, similar to what was done when checking the 
residuals (again, only single-site events are checked). When calculating the correlation matrices, the systematic errors estimated from the shape agreement (see section 4.3) are propagated using a binned approximation in order to reduce compuation time. In all correlation matrices, the lowest diagonal elements are omitted due to diagonal symmetry.

The correlation matrices for ${ }^{228} \mathrm{Th}$ were found to be,

${ }^{228}$ Th data $=\left[\begin{array}{ccc}\text { Standoff Distance } & \text { Cluster Risetime } & \text { Collection Wires } \\ 1 & 0.0013 \pm 0.0022 & 0.0692 \pm 0.0011 \\ 1 & 0.166 \pm 0.015 \\ & & 1\end{array}\right]$ Collection Wires

and,

${ }^{228} \mathrm{Th} \mathrm{MC}=\left[\begin{array}{ccc}\text { Standoff Distance } & \text { Cluster Risetime } & \text { Collection Wires } \\ 1 & 0.0307 \pm 0.0047 & 0.0643 \pm 0.0018 \\ & 1 & 0.178 \pm 0.26 \\ & & 1\end{array}\right]$ Collection Wires

where the greatest discrepancies are in the standoff distance/collection wires and standoff distance/cluster risetime coefficients. These differences were larger than the systematic error ( $2.3 \sigma$ and $5.7 \sigma$ respectively) so the effects of any disagreement in the $\mathrm{BDT}$ result were investigated (see section 4.5.3). The correlation matrices for $2 \nu \beta \beta$ were found to be, 


$2 \nu \beta \beta$ Data $=\left[\begin{array}{ccc}\text { Standoff Distance } & \text { Cluster Risetime } & \text { Collection Wires } \\ 1 & 0.0133 \pm 0.0014 & 0.060 \pm 0.023 \\ 1 & 0.13 \pm 0.37 \\ & & 1\end{array}\right]$ Collection Wires

and,

$2 \nu \beta \beta \mathrm{MC}=\left[\begin{array}{ccc}\text { Standoff Distance } & \text { Cluster Risetime } & \text { Collection Wires } \\ 1 & 0.03617 \pm 0.00026 & 0.0740 \pm 0.0041 \\ 1 & 0.121 \pm 0.066 \\ & & 1\end{array}\right]$ Collection Wires

where now the greatest discrepancy is that of the standoff distance/cluster risetime coefficient. This difference is even larger than the previous $(16.4 \sigma)$ and thus any disagreement between the data and $\mathrm{MC}$ of the discriminator must be carefully examined.

The BDT is now trained using on Monte Carlo selecting 1000000 events each for training and testing. Once the BDT is built, it is applied to all necessary data and Monte Carlo so that the same checks can be done to the BDT discriminator that were done to each input variable (i.e. shape agreement and separation). When tested for overtraining using the K-S test, a p-value >0.999 was found between the training and testing data sets, indicating a $>99.9 \%$ probability that both sets follow the same distribution (i.e. the BDT is not overtrained). The data/MC agreement for the BDT discriminator are presented in figure 4.16 where nearly all bins show $\mid$ res $\mid<0.1$ with the exception of a few low statistic bins. This is the high level check to see that our BDT is unbiased and the result is satisfactory.

Figure 4.17 shows the discriminator distributions for $0 \nu, 2 \nu$ and background events. We now calculate a separation score of $\sim 0.32$ which is an order of magnitude 


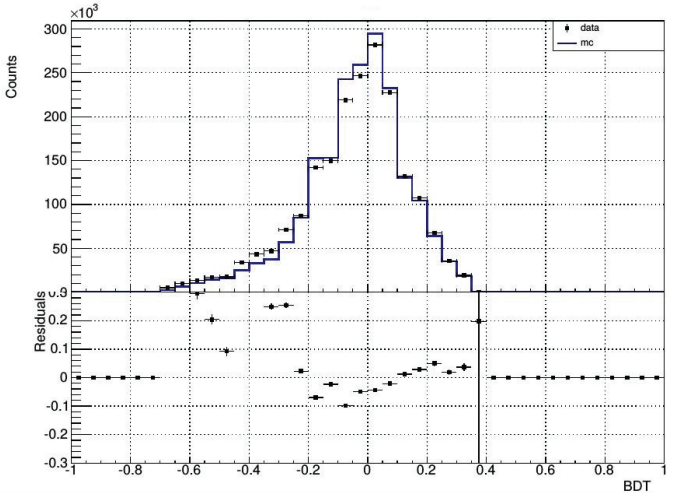

(a) ${ }^{228}$ Th shape agreement.

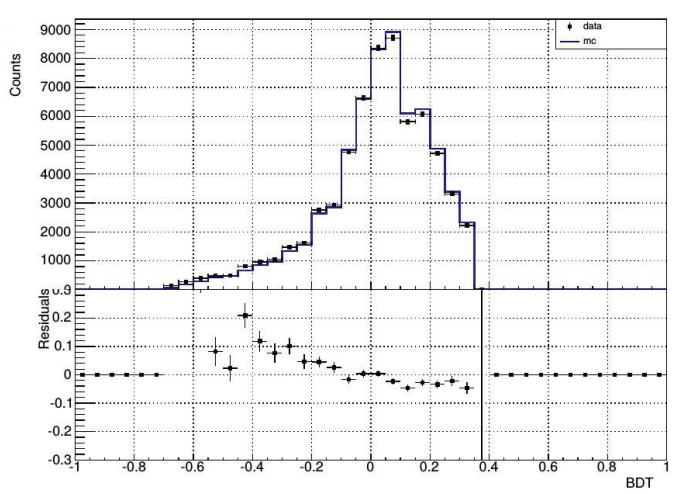

(b) $2 \nu \beta \beta$ shape agreement.

Figure 4.16: BDT discriminator shape agreement showing event distributions (upper) and residuals (lower) for both ${ }^{228} \mathrm{Th}$ and $2 \nu \beta \beta$ events.

improvement over any of the input variables on their own.

\subsection{Analysis Process}

The physics analysis of EXO-200 data is performed in several stages all managed by the EXO-200 fitting package which utilizes RooFit [36] workspaces for all data handling and fitting processes. In the first stage of the analysis, all data is collected together into a number of datasets, one set for real physics data and one set for each Monte Carlo component (all background components as well as $0 \nu \beta \beta$ signal). The datasets are then subjected to a series of cuts to produce three entry lists, one for each data and MC (signal/background) dataset. The cuts applied separate the events into lists of single-site and multi-site events (plus a third list containing both), remove events outside the hexagonal fiducial volume and remove events with missing information or which are poorly reconstructed (generally problematic events). A further selection is made which keeps events only within the variable ranges over which the fit will be performed (in the Nature publication $980 \mathrm{keV}<$ energy $<9800$ keV [16], now $1 \mathrm{MeV}<$ energy $<10 \mathrm{MeV}$ and $-1<$ discriminator $<1$ ).

Once the entry lists have been created, a set of 2-D (energy vs. discriminator) binned RooFit PDFs are generated for each list. These PDFs act as normalized histograms which describe the probability of finding an event in a given bin for any given signal/background component. Separate PDFs are produced for SS/MS events. 


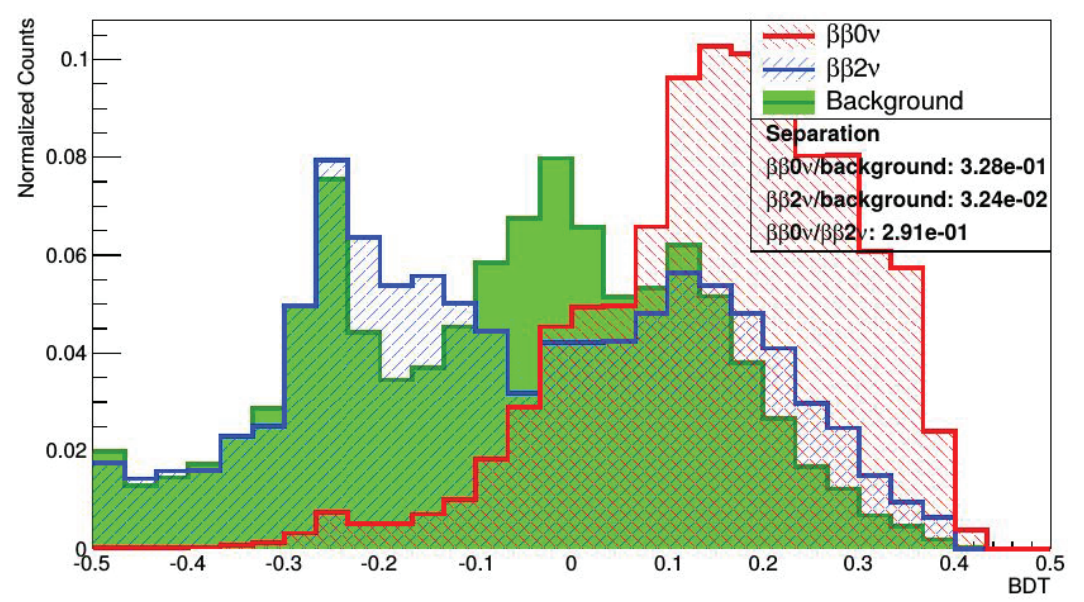

Figure 4.17: BDT discriminator distributions for $0 \nu \beta \beta, 2 \nu \beta \beta$ and background events with calculated separation scores between each.

Once all PDFs have been created, they are combined into two main PDFs which represent the overall distribution of SS and MS events in the TPC. These PDFs contain a number of floating parameters which will be determined later. The combined PDFs take the form,

$$
P_{\text {Total }}^{\mathrm{SS}}=n_{\text {all }}\left(P_{\text {standard }}^{\mathrm{SS}}+P_{\mathrm{nCap}}^{\mathrm{SS}}\right)
$$

where,

$$
P_{\text {standard }}^{\mathrm{SS}}=\sum_{i=0}^{N_{\mathrm{std}}} n_{i} \cdot f_{i} \cdot P_{i}^{\mathrm{SS}}
$$

and,

$$
P_{\text {nCap }}^{\mathrm{SS}}=n_{\mathrm{nCap}} \sum_{i=0}^{N_{\text {nCap }}} f_{i}^{\mathrm{nCap}} \cdot f_{i} \cdot P_{i}^{\mathrm{SS}}
$$

where $P_{i}^{\mathrm{SS}}$ are the individual single-site PDF components, $n_{i}$ is the number of events of the $i$-th component and $f_{i}$ is the fraction of these events which are single-site (the MS version of the PDF is similar, simply replacing $f_{i}$ with $\left.\left(1-f_{i}\right)\right)$. The neutron capture components of the PDF are treated slightly different in that there is just one parameter, $n_{\mathrm{nCap}}$, which represents the total number of neutron capture events and then $f_{i}^{\text {nCap }}$ is the fraction of these events coming from the $i^{\text {th }}$ component. There are also two hidden parameters, $n_{\mathrm{ROI}}$ and $\beta$, which along with the normalization factor, 
$n_{\text {all }}$, and the single-site fractions are used to incorporate systematic errors into the fit. These "nuisance" parameters will be discussed in more detail later in section 4.5.3.

A full list of PDF components can be found in table 4.3. Within this list, different PDF components are grouped into either standard or neutron capture based on which type of PDF weighting they use and are coloured based on how they are grouped when plotting the fit results and bolded elements represent background components with major contributions in the $2 \sigma$ ROI (the region containing $95.45 \%$ of all $0 \nu \beta \beta$ events, 2383 to $2533 \mathrm{keV}$ ). They are further also separated into subgroups which generally describe either the location or nature of the event type. The "Vessel" subgroup contains background components consisting of either radioactive contamination or cosmogenically activated isotopes within the copper contituting the TPC vessel. The "Radon" subgroup contains ${ }^{222} \mathrm{Rn},{ }^{214} \mathrm{~Pb}$ and ${ }^{214} \mathrm{Bi}$ (both members of the ${ }^{222} \mathrm{Rn}$ decay chain) at various locations around the detector. During the fitting process, the radon components within the xenon are constrained to within $10 \%$ of the values measured during a standalone study of radon backgrounds. The "Active LXe" subgroup contains non- ${ }^{136} \mathrm{Xe} \beta \beta$ events which occur within the LXe. The "Neutron Capture" group/subgroup contains all types of neutron capture events which can occur in the HFE, the LXe or in the copper vessel/cryostat. Similar to the radon subgroup, the neutron capture components are constrained to within $20 \%$ of levels established by a standalone study. Finally the "Other" subgroup contains background components which did not fit anywhere else, specifically the $2 \nu \beta \beta$ background, ${ }^{232}$ Th contamination in the inner cryostat and some ${ }^{60} \mathrm{Co}$ contamination in the tube through which the calibration sources are deployed.

Once built, the PDFs can be simultaneously fit to the SS/MS EXO-200 datasets in order to determine all of the aforementioned parameters. This is done using a negative-log-likelihood fit. The likelihood function is related to the probability density function (PDF) in the sense that, the PDF describes the odds of an outcome for a given set of parameters whereas the likelihood function describes the odds that a set of parameters could produce a given outcome. More simply put,

$$
\mathcal{L}(\theta \mid \mathbf{x})=P(\mathbf{x} \mid \theta)
$$

where $\mathcal{L}$ is the likelihood function and $P$ is the PDF for a set of parameters, $\theta$ and a set of outcomes, $\mathbf{x}$. It is intuitive that by maximizing the likelihood function the 
Table 4.3: All components of the full EXO-200 PDF along with the corresponding fit result for their $n_{i}$ parameter.

\begin{tabular}{|c|c|c|c|}
\hline Group & Sub-group & Name & Fit Result (cts) \\
\hline Signal & Signal & $\mathrm{bbOn}$ & 16.556 \\
\hline \multirow{4}{*}{$\begin{array}{c}\text { Standard } \\
\text { Backgrounds }\end{array}$} & Other & $\begin{array}{c}\text { bb2n } \\
\text { InnerCryo_Th232 } \\
\text { SourceTube_Co60 }\end{array}$ & $\begin{array}{c}54854 \\
850.92 \\
3.4084 \times 10^{-5}\end{array}$ \\
\hline & Vessel & $\begin{array}{c}\text { AllVessel_U238 } \\
\text { AllVessel_Zn65 } \\
\text { AllVessel_Th232 } \\
\text { AllVessel_Co60 } \\
\text { AllVessel_K40 }\end{array}$ & $\begin{array}{l}2016.8 \\
146.84 \\
1186.8 \\
2555.5 \\
6691.8\end{array}$ \\
\hline & Radon & $\begin{array}{c}\text { InactiveLXe_Rn222 } \\
\text { CathodeSurf_Bi214_nochain } \\
\text { ActiveLXe_Pb214_only } \\
- \text { AirGap_214_Bi_nochain }\end{array}$ & $\begin{array}{c}168.10 \\
--------- \\
726.00\end{array}$ \\
\hline & Active LXe & $\begin{array}{l}\text { ActiveLXe_Xe137 } \\
\text { ActiveLXe_Xe135 }\end{array}$ & $\begin{array}{l}107.20 \\
432.42\end{array}$ \\
\hline $\begin{array}{l}\text { Neutron } \\
\text { Capture }\end{array}$ & $\begin{array}{l}\text { Neutron } \\
\text { Capture }\end{array}$ & $\begin{array}{c}\text { nCap_LXe_randE } \\
\text { nCap_InnerCryo_Cu63 } \\
\text { nCap_InnerCryo_Cu65 } \\
\text { nCap_OuterCryo_Cu63 } \\
\text { nCap_OuterCryo_Cu65 } \\
\text { nCap_Vessel_Cu63 } \\
\text { nCap_Vessel_Cu65 } \\
\text { nCap_HFEr1_H1 } \\
\text { nCap_HFEr2_H2 } \\
\text { nCap_HFEr1_H3 } \\
\text { nCap_HFEr1_H4 } \\
\text { nCap_HFEr1_H5 } \\
\text { nCap_HFEr1_H6 }\end{array}$ & 210.95 \\
\hline
\end{tabular}


set of parameters most likely to result in a given data set can be determined. This is the basis behind the negative-log-likelihood fit. If the vector $\mathbf{x}$ is expanded into it's individual components, $\mathbf{x}=\left(x_{0}, x_{1}, \ldots, x_{N}\right)$, the likelihood function takes the following form,

$$
\mathcal{L}(\theta \mid \mathbf{x})=\prod_{i=0}^{N} p\left(x_{i} \mid \theta\right)
$$

It is clear from equation 4.15 that when calculated for a large dataset the likelihood function will very quickly converge to 0 (product of many real positive numbers less than 1) making this difficult to compute. A simple solution to this problem is to use the log-likelihood,

$$
\log \mathcal{L}(\theta \mid \mathbf{x})=\sum_{i=0}^{N} \log \left(p\left(x_{i} \mid \theta\right)\right)
$$

which will now be a negative number of larger magnitude. Because the function $\log (z)$ is always increasing with $z$, maximizing the log-likelihood will simultaneously maximize the likelihood. This process is typically taken one step farther by using $-\log \mathcal{L}$. The fit is now a minimization problem which can be easily handled by ROOT's MINUIT minimizer [35]. It should be noted that because a binned fit is used, the negative-log-likelihood (NLL) takes the form,

$$
-\log \mathcal{L}(\theta \mid \mathbf{x})=-\sum_{i=0}^{N} N_{i} \cdot \log \left(p\left(x_{i} \mid \theta\right)\right)
$$

where $N_{i}$ is the number of events in the $i^{t h}$ bin and $x_{i}$ is now the centre of the bin. In the case of the EXO-200 analysis, $\theta$ is the set of parameters previously explained and each $x_{i}$ is a vector containing the centre coordinates of each bin in energy vs. discriminator. Furthermore there are two terms in the sum, one for the SS likelihood and one for the MS likelihood. The $\theta$ used in each term is the same, however the SS term will only use $x_{i}$ terms corresponding to SS bins and similarly for the MS. By varying the configuration of this fit (by fixing or releasing various parameters), several useful results can be obtained including the number of $0 \nu \beta \beta$ events. The results of using this analysis technique for the full Phase-I data set can be seen in figure 4.18.

In order to achieve a more detailed result, the parameter $n_{0 \nu \beta \beta}$ (written as 

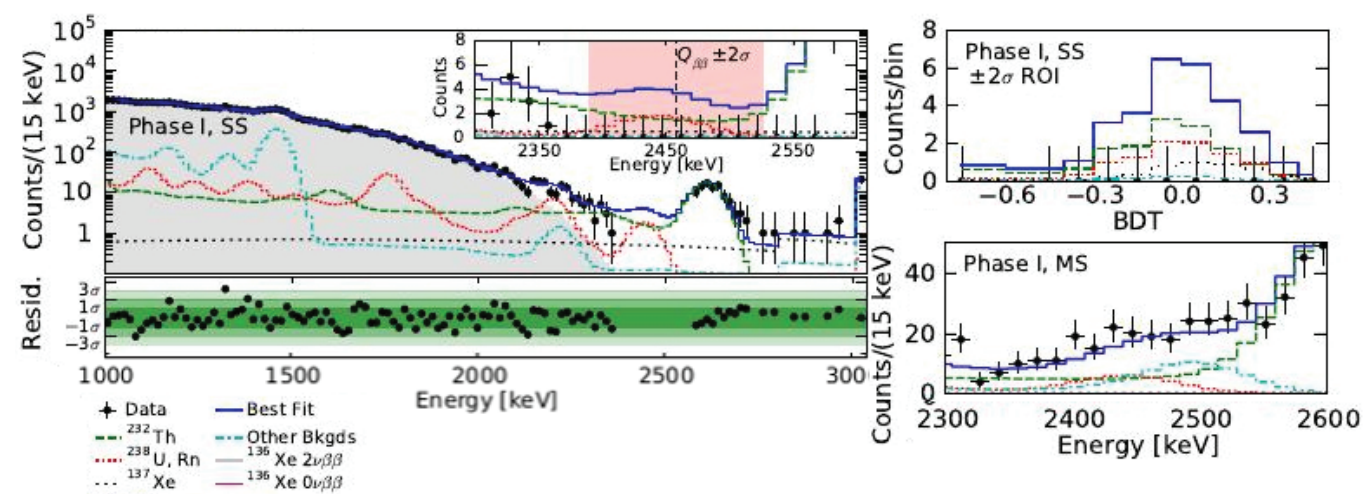

Figure 4.18: Fit result using the new analysis technique showing 1-D projections into energy for SS (with ROI inset) and MS events (only ROI shown) and BDT discriminator for SS only. Results are shown for fit to full Phase-I data set. Background components in the fit are grouped according to their colors in table 4.3. Adapted from [37].

num_bb0n), representing the number of $0 \nu \beta \beta$ events is manually profiled. To profile $n_{0 \nu \beta \beta}$, its value is set to a constant hypothesis and all other fit parameters are minimized. This is done for a number of values about the minimum NLL to create a curve, called the profile, of NLL vs. $n_{0 \nu \beta \beta}$. The NLL profile over $n_{0 \nu \beta \beta}$ is shown in figure 4.19 for the full Phase-I data set using this new analysis technique along with two other analysis techniques for comparison. Wilks theorem [38] tells us that with a sufficient number of events, the $\Delta$ NLL follows a $\chi^{2} / 2$ distribution allowing us to set confidence intervals on the NLL profile (in this case $90 \%$ CI at $\sim 1.35$ ). This upper limit on the number of $0 \nu$ events can be converted into a lower limit on the decay half-life or even taken a step further to calculate a neutrino mass upper limit.

\section{$0 \nu \beta \beta$ Half-Life Lower Limit and $\left\langle m_{\beta \beta}\right\rangle$ Upper Limit}

Once an upper limit on the number of $0 \nu$ events has been calculated, it is straightforward to convert this into a lower limit on the decay half-life. This is done via equation 4.18 ,

$$
T_{1 / 2}^{0 \nu}=\frac{M_{\mathrm{Xe}} \cdot N_{\mathrm{Xe} / \mathrm{kg}} \cdot \xi \cdot t \cdot \log 2}{\left(N_{\text {upper }} / \epsilon\right)}
$$

where $M_{\mathrm{Xe}}$ is the fiducial mass of xenon, $N_{\mathrm{Xe} / \mathrm{kg}}$ is the number of xenon atoms per $\mathrm{kg}$, $\xi$ is the enrichment fraction of ${ }^{136} \mathrm{Xe}, t$ is the detector livetime, $N_{\text {upper }}$ is the upper 


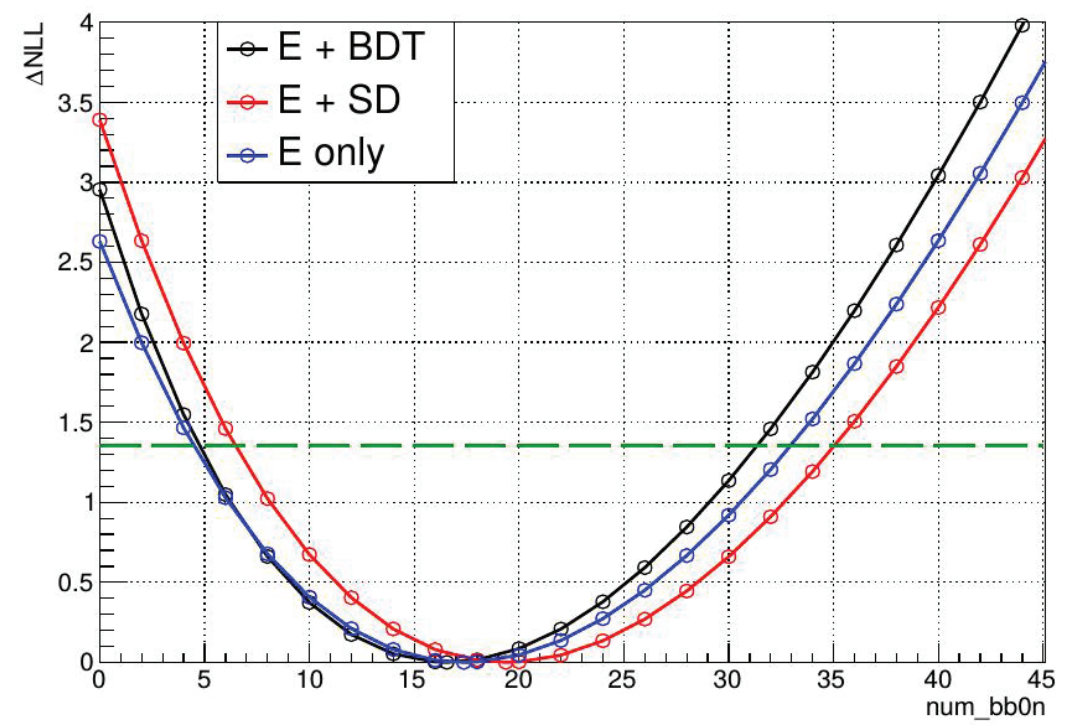

Figure 4.19: The NLL profile for the number of $0 \nu \beta \beta$ events. Profiles are shown for the Energy + BDT fit detailed in this work as well as the baseline Energy + Standoff Distance fit and a 1D Energy only fit. The 90\% confidence level (assuming the validity of Wilk's theorem) is indicated by the dashed horizontal green line.

limit of the number of $0 \nu \beta \beta$ events and $\epsilon$ is the detector efficiency. As discussed in chapter 2, the effective Majorana mass upper limit can then be calculated from the half-life limit via,

$$
\left\langle m_{\beta \beta}\right\rangle=\frac{m_{\mathrm{e}}}{M^{0 \nu}} \sqrt{\frac{1}{T_{1 / 2}^{0 \nu} G^{0 \nu}}}
$$

where $G^{0 \nu}$ is the leptonic phase space factor which can be calculated precisely, $M^{0 \nu}$ is a nuclear matrix element which carries a large theoretical error and $m_{\mathrm{e}}$ is the electron mass.

\subsubsection{Sensitivity}

In a counting experiment, the sensitivity is defined as the most likely statistical limit that an experiment will put on an unobserved effect. In the case of EXO-200, this is the most likely upper limit the experiment will place on the number of $0 \nu \beta \beta$ events (and therefore the Majorana-neutrino mass) in the event that none are detected. In a counting experiment, typically a region of interest (ROI) is defined in which the 
goal of the experiment is to count the number of events from some type of signal. In any realistic experiment, this region of interest will also contain some number of background events. If we define the mean number of signal events as $s$ and the mean number of background events as $b$, then the total number of events $N$ observed in the ROI follows a Poisson distribution with mean $\mu=s+b$ with PDF given by,

$$
P(N \mid \mu)=\frac{\mu^{N}}{N !} e^{-\mu}
$$

Rewriting this in terms of $s$ and $b$, we define a distribution for the number of signal events observed, $s^{\prime}$,

$$
P\left(s^{\prime} \mid s+b\right)=\frac{(s+b)^{s^{\prime}+b}}{\left(s^{\prime}+b\right) !} e^{-(s+b)}
$$

where we have now substituted $N \rightarrow s^{\prime}+b$. For a given dataset following this distribution, an upper limit on s can be calculated. The method of calculation may vary between experiments, however for EXO-200, a negative-log-likelihood profiling method was used (as previously discussed). Were the experiment to be repeated, it would be seen that the upper limits follow some distribution $h\left(s_{\text {up }} \mid \mu\right)$. The sensitivity, $\tilde{s}_{\text {up }}$, of the experiment is then by definition,

$$
0.5=\sum_{s_{\mathrm{up}}=0}^{\tilde{s}_{u p}} h\left(s_{u p} \mid b\right)
$$

since half of all calculated upper limits fall below this value and half fall above, this is also known as the median of the distribution of upper limits.

In order to calculate the detector sensitivity, a background model must be constructed. This is done by performing a NLL fit as previously described while fixing $n_{0 \nu \beta \beta}$ to 0 . When performing this fit, the extra fit parameters $n_{\text {all }}, n_{\mathrm{ROI}}$ and $\beta$ are not used (the use of these parameters will be explained later in section 4.5.3). The result of this fit is a background model in which it is assumed there are no $0 \nu \beta \beta$ events. It is important when comparing different analysis techniques that the same background model is used for each since the number of background counts has a strong impact on the sensitivity.

This background model is used to generate a large number $(\sim 5000)$ of toy MC data sets. A NLL fit is performed on each toy (now including $n_{0 \nu \beta \beta}$ and the other 


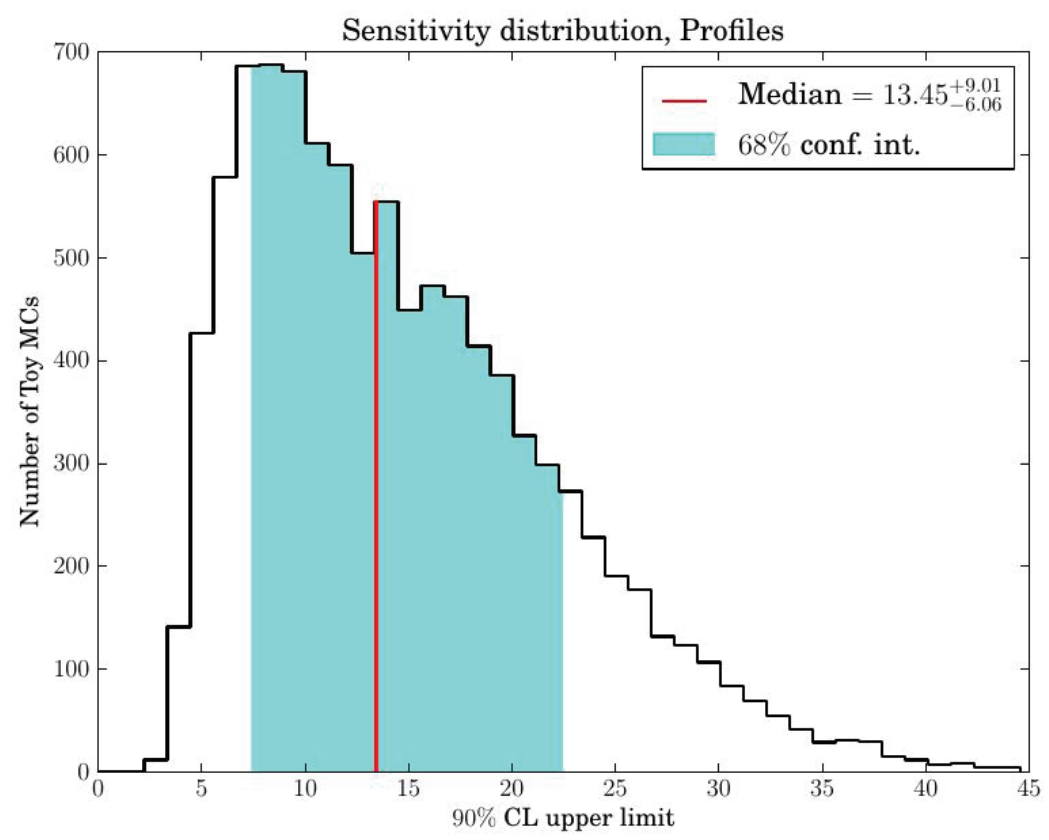

Figure 4.20: A histogram of the $90 \%$ CL upper limits on the number of $0 \nu \beta \beta$ events calculated on 5000 toy data sets. The vertical red line indicates the median upper limit corresponding to the sensitivity of the experiment $(13.45$ $0 \nu \beta \beta$ events).

extra parameters) and $n_{0 \nu \beta \beta}$ is profiled to find the $90 \%$ confidence level (CL) upper limit on each. The median 90\% CL upper limit is taken to be the sensitivity of the experiment and is subsequently converted to a half-life using equation 4.18. An example histogram of $90 \%$ CL upper limits used in sensitivity calculations is shown in figure 4.20. The example shown uses a 1-D energy-only analysis technique and results in a sensitivity of $13.450 \nu \beta \beta$ events corresponding to a half-life sensitivity of $2.37 \times 10^{25}$ years.

\subsubsection{Discovery Potential}

The discovery potential of an experiment is conceptually similar to the sensitivity in that it is a statistical method of evaluating detector performance. Unlike the sensitivity however, this test calculates the significance level of a discovery using a model which includes signal. This test stems from the likelihood ratio test assuming the validity of Wilk's theorem [38] where a test statistic, t, given by,

$$
t=-2 \log \Lambda
$$


follows a $\chi^{2}$ distribution. Here $\Lambda$ is the likelihood ratio between the null hypothesis $\left(n_{0 \nu \beta \beta}=0\right.$ and a chosen alternate hypothesis $\left(n_{0 \nu \beta \beta}>0\right)$. This test statistic can be rewritten by expanding $\Lambda$,

$$
\begin{aligned}
t & =-2 \log \frac{\mathcal{L}_{0}}{\mathcal{L}_{1}} \\
& =2\left(-\log \mathcal{L}_{0}+\log \mathcal{L}_{1}\right) \\
& =2\left(N L L_{0}-N L L_{1}\right) \\
& =2 \cdot \Delta N L L
\end{aligned}
$$

In order to calculate the discovery potential, again a background model is constructed. This background model is then fit with two different PDFs, one with fixed $n_{0 \nu \beta \beta}=0$ and one which allows for $n_{0 \nu \beta \beta}>0$. The difference is then calculated between the minimum NLL values to find $\triangle$ NLL which is then converted to the test statistic, t. This process is repeated for a large number of toy $\mathrm{MC}$ and the distribution of $t$ is plotted.

A similar process is repeated, this time using a model which includes a $0 \nu \beta \beta$ signal corresponding to $T_{1 / 2}^{0 \nu}=2.6 \times 10^{25} \mathrm{yr}$, the central value of the Nature analysis [16]. The $t$-distribution using the signal model is then superimposed on that of the backgroundonly model. A discovery probability, $\beta$, is selected for the discovery potential (i.e. 0.50 or 0.90 ) representing the probability that given an ensemble of experiments, $\beta$ is the fraction of them that will register a discovery at the given significance level. The $1-\beta$ percentile of the signal model distribution is then found (i.e. the value of $t$ for which $\beta \cdot 100 \%$ of the distribution lies above this value). The discovery potential at the chosen significance level is then the fraction of $t$ values, $\alpha$, in the background-only distribution falling above this value. This process is illustrated in figure 4.21.

\subsubsection{Systematic Uncertainty}

Systematic uncertainties in the analysis technique are incorporated by introducing a number of nuisance parameters to the PDFs used in the NLL fit. By adding these variables, the fit is relaxed, reducing the NLL. This effectively broadens the NLL profile, increasing (worsening) the upper limit on $n_{0 \nu \beta \beta}$. These parameters are $n_{\text {all }}$, $n_{\mathrm{ROI}}, \beta$ and all of the single site fraction parameters $\left(f_{i}\right)$. 


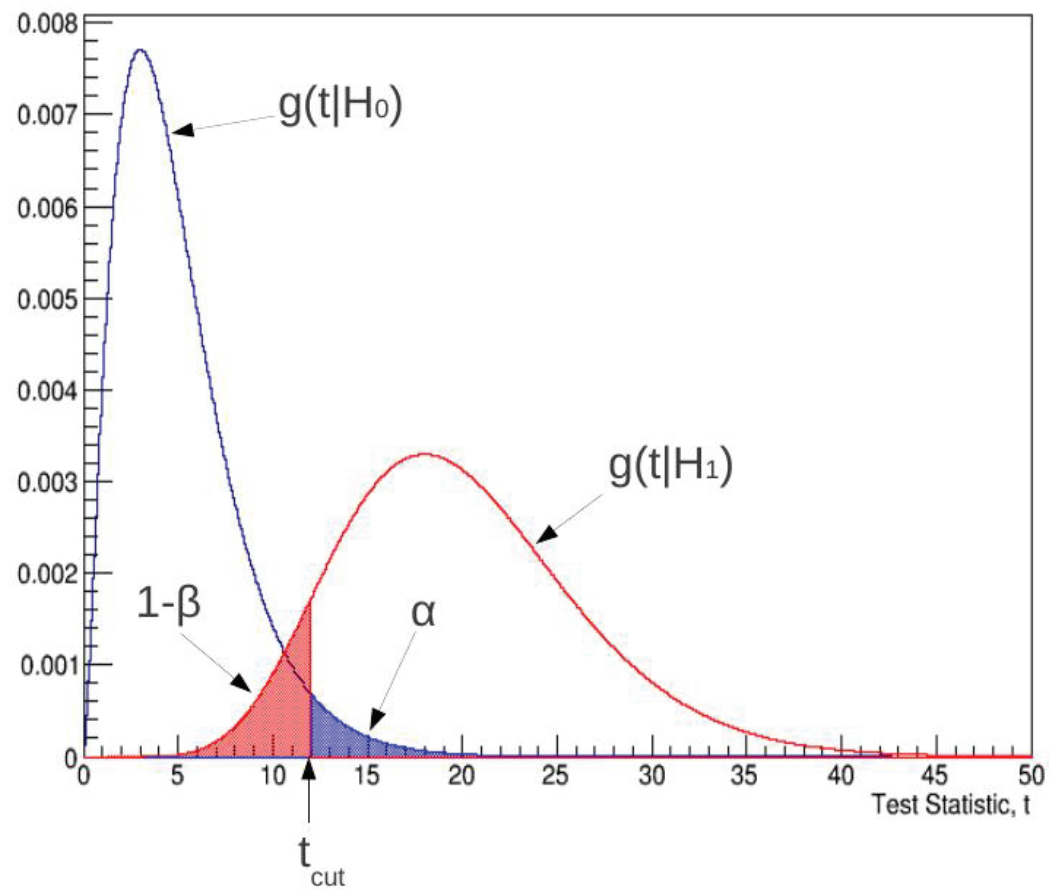

Figure 4.21: The distribution of the test statistic, $t$, for the null hypothesis and an alternate hypothesis. Reprinted from [39].

The normalization parameter, $n_{\text {all }}$, represents the uncertainty on the event detection efficiency. The factor combines uncertainties contributed by some event selection, 3D event reconstruction efficiency and fiducial volume agreement. For the analysis shown in [16] the detection efficiency error was 8.6\%. This has now been reduced to $3.0 \%$ for the new Phase-I analysis, corresponding to $n_{\text {all }}=1 \pm 0.030$.

As mentioned in section 4.3, the residuals calculated between data and MC are used to estimate systematic error. The propagation of this uncertainty into the NLL fit is done through toy MC studies which allow us to estimate appropriate gaussian constraints on another nuisance parameter. The original PDFs of each background/signal components are unskewed (i.e. modified) by the calculated residual errors to create new unskewed PDFs ( $\gamma$ components are modified using residuals from the ${ }^{228}$ Th calibration source, $\beta$ components are modified using residuals from background subtracted $2 \nu \beta \beta$ data). These unskewed PDFs are then used to generate a large number of simulated data sets following the best-fit background-only model, the results of which are then fit using the original, un-modified PDFs. The fitted number of events within the $2 \sigma$ ROI is compared to the original best fit and the difference is incorporated as a nuisance normalization parameter within the ROI. The 
ROI normalization parameter, $n_{\mathrm{ROI}}$, represent the error on the background model within the $2 \sigma$ region of interest (ROI) arising from shape differences between data and MC. The ROI normalization is included in the fit by splitting the PDFs of each major ROI background component (denoted in table 4.3 by a *) into separate PDFs, one for the ROI, one for the remaining energy range. The PDFs contributing to the ROI $(\mathrm{U}, \mathrm{Th}, \mathrm{Xe}, \mathrm{Co})$ are then rescaled by a factor of $\left(1+n_{\mathrm{ROI}}\right)$. For the new Phase-I analysis the ROI normalization error was found to be $6.2 \%$, corresponding to $n_{\text {ROI }}=0 \pm 0.062$.

The $\beta$ parameter attempts to compensate for uncertainty on the energy calibration for $\beta$ events. This was introduced because the calculation of the rotated energy scale is done entirely using $\gamma$ sources with no direct $\beta$ source calibration. The $\beta$ factor rescales the energy of all $\beta$ PDFs and is allowed to float completely free (can take any value in the fit). When studied, $\beta$ was found to be consistent with unity to better than $0.7 \%$.

Finally, the single-site fraction parameters all carry an associated error. The initial values of these parameters are set from the simulated SS-fraction obtained from Monte Carlo. While the initial value is different for each component, the error on each for Phase-I is set to $5 \%$ which was determined by comparing calibration source data to the corresponding Monte Carlo.

\subsection{Results}

Using the techniques detailed in the previous sections, the sensitivity and discovery potential have been calculated using three different analysis techniques. The first technique is a 1-D energy-only fit, this technique has not previously been used for an official analysis however it is interesting for use in comparison to see what improvement the standoff distance offers. The second technique is a 2-D fit of energy vs. standoff distance. This is the analysis technique used in [16] and is the baseline to which the new analysis must be compared. The final technique is the 2-D energy vs. BDT discriminator fit which has been explained throughout this chapter. Table 4.4 summarizes the results obtained using these three techniques with the EXO-200 full Phase-I dataset. It is important to note that the results shown here were calculated concurrently with the new EXO-200 results. The final published results may change slightly however the relative improvement will remain the same. 
Table 4.4: Results of EXO-200 analysis using full Phase-I dataset comparing the new 2D energy vs. BDT discriminator fit to the previously used energy vs. standoff distance fit as well as a 1D energy-only fit.

\begin{tabular}{|c|c|c|c|}
\hline Result & E-only & E + SD & E + BDT \\
\hline \hline \multicolumn{3}{|c|}{ Monte Carlo Driven Results } \\
\hline $\begin{array}{c}\text { Sensitivity } \\
\left(\times 10^{25} \text { years }\right) \\
\text { Discovery Potential }\end{array}$ & 2.6 & 2.6 & 2.9 \\
\hline (p-value \% for $T_{1 / 2}^{0 \nu}=2.6 \times 10^{25}$ years $)$ & 4.9 & 5.1 & 3.5 \\
\hline \multicolumn{3}{|c|}{ Data Driven Results } \\
\hline \begin{tabular}{c|c}
$n_{\mathrm{bb} 0 \mathrm{n}}($ counts $)$ \\
$T_{1 / 2}^{0 \nu}\left(\times 10^{24}\right.$ years $)$
\end{tabular} & $<33$ & $<35$ & $<31$ \\
$\left\langle m_{\beta \beta}\right\rangle(\mathrm{meV})^{1,2}$ & $>9.4$ & $>8.8$ & $>9.9$ \\
$<203-551$ & $<210-569$ & $<198-537$ \\
\hline
\end{tabular}

\footnotetext{
${ }^{1}$ Variation is due to different choice of model used to calculate $M^{0 \nu}$.

${ }^{2}$ Values of $M^{0 \nu}$ and $G^{0 \nu}$ are the same used in [37].
}

Table 4.4 also contains a new lower limit on the $0 \nu \beta \beta$ half lifetime in ${ }^{136} \mathrm{Xe}$ calculated using this new analysis technique on the full Phase-I data set. This new limit is $\sim 10 \%$ lower than the limit found in [16] (see table 4.1) and shows a $\sim 13 \%$ improvement over the baseline Energy + Standoff Distance technique. The reduction of the lower limit relative to the Nature result could be due to a number of factors (e.g. larger number of background counts due to increased livetime, more conservative error estimation, etc.), the important thing is the comparison between the new technique and the old. New limits are also presented for the effective Majorana-neutrino mass for completeness however due to the large variation based on the calculation of the nuclear matrix elements (NMEs), the limit on $T_{1 / 2}^{0 \nu}$ remains the focal point of these results.

The improvements to the sensitivity, discovery potential and half-lifetime lower limit using the new analysis technique are clear. The 2-D energy vs. BDT discriminator fit brings a $\sim 12 \%$ improvement to sensitivity over the baseline energy vs. standoff distance fit. Furthermore it also brings about a $31 \%$ improvement to the discovery potential and a $\sim 13 \%$ improvement to the $T_{1 / 2}^{0 \nu}$ lower limit. The use of this analysis 
technique has been approved for further use in future EXO-200 analyses including the upcoming analysis of the full Phase-I and II datasets. 


\section{Chapter 5}

\section{Ba Tagging}

In section $3.2 \mathrm{I}$ briefly introduced the concept of barium tagging for use in nEXO. By identifying the ${ }^{136} \mathrm{Ba}^{++}$daughter ion produced in the double beta decay of a

${ }^{136} \mathrm{Xe}$ atom, it would be possible to eliminate all non- $\beta \beta$ background events in the detector and in particular eliminate virtually all background events in the $2 \sigma$ region of interest (i.e. the region within which $95.45 \%$ of all $0 \nu \beta \beta$ events occur). This is obviously desirable as the reduction of background would lead to a significant increase in sensitivity. Because only a few $0 \nu \beta \beta$ events are expected per year, even a small amount of background event can easily cause the signal to become lost in noise (following a Poisson distribution, noise $\propto \sqrt{n}$ ). In both experiments, the vast majority of backgrounds in the ROI are gammas from the ${ }^{232} \mathrm{Th}$ and ${ }^{238} \mathrm{U}$ decay chain. By tagging barium virtually all of these backgrounds would be eliminated, leading to a large increase in sensitivity.

In this chapter, I will go on to describe the experimental setup as well as the underlying physics of the barium tagging apparatus that currently exists at Carleton. I will then describe my work which led to improvements to the system and present new barium tagging results which I will compare to those previously demonstrated in [19]. Furthermore, I will describe my work towards using this system to tag not only barium, but individual barium isotopes.

Although work is in progress at various institutions around the world to study barium tagging in solid or gas phase, this study focuses on barium tagging in gas. Because $\mathrm{nEXO}$ is a liquid TPC, tagging in gas first requires that the barium ion is extracted from the liquid xenon. A complete system for barium tagging in gas would consist of three main components: extraction of $\mathrm{Ba}^{++}$and LXe from the TCP (and conversion to gas phase), extraction of $\mathrm{Ba}^{++}$from gaseous xenon and finally trapping 
and identification of $\mathrm{Ba}^{+}$. While the first two components are in development at other locations, this work focuses primarily at the third component, the trapping and identification (or tagging) of the $\mathrm{Ba}^{+}$ion. The original work presented in this chapter focuses primarily on improvements to the ion trapped and optical tagging systems currently in use.

Each of these stages has already been studied and working systems have been demonstrated by nEXO collaborators at various institutions. Extraction of ions from a high pressure region to a low pressure region via an RF funnel has been demonstrated at Stanford University [40]. The trapping and identification of ions has been demonstrated both at Stanford [13,41] and Carleton Universities [19] and charge conversion of ${ }^{136} \mathrm{Ba}^{++}$to ${ }^{136} \mathrm{Ba}^{+}$has been demonstrated at Carleton University [42]. A system is also under development now for the extraction of barium from LXe using a cryogenic probe. A sketch of the planned overall barium tagging scheme is shown in figure 5.1 indicating the institutions at which each component is being developed and tested. This diagram also depicted a newer component of the system, a MultiReflection Time of Flight (MR-TOF) Mass Spectrograph, which will be used as a secondary detection phase after the optical tagging. In the figure 5.1, the ion trap is attributed to TRIUMF. This will be a third generation ion trap to replace the one currently in use and will be discussed further near the end of this chapter. The ion trap which will be discussed in detail in this chapter was developed at Stanford University and has been thouroughly tested at both Stanford and Carleton.

\subsection{Linear Ion Trap}

In order to trap and confine barium ions, a linear quadrupole ion guide with a confining longitudinal potential is used. At a basic level, this consists of four parallel cylindrical rods arranged symmetrically about a central axis (see figure 5.2). A radiofrequency $(\mathrm{RF})$ potential (of the form $\pm V \cos \Omega t$, where $V$ is the amplitude of the potential and $\Omega$ is the frequency of oscillation) on the rods confines ion radially while a z-dependent potential well confines them axially. The underlying physics and experimental setup of the trap will be explored in depth in the following sections. 


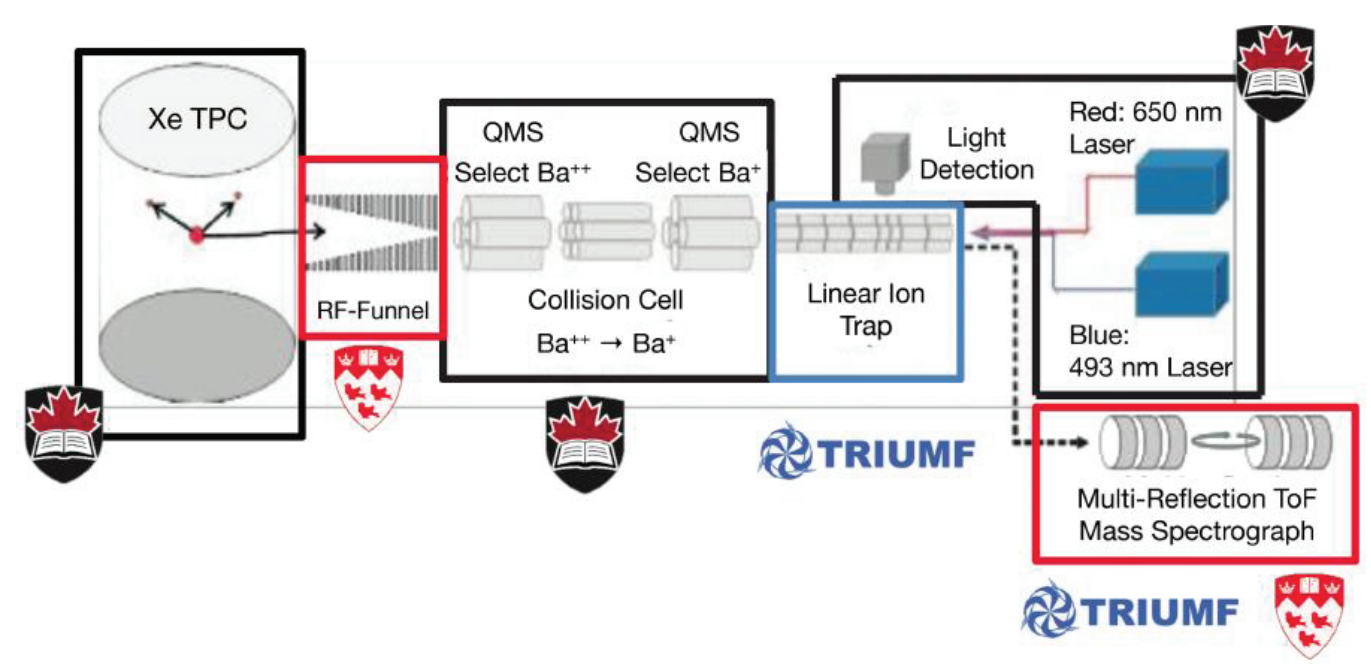

Figure 5.1: A diagram of the planned barium tagging scheme. A barium ion is extracted from the liquid xenon TPC via a cryogenic probe at which point it is evaporated toward the entrance of an RF funnel. This funnel then removes any gaseous xenon allowing the ion to be trapped and optically tagged in vacuum. Also shown is an MR-TOF system which will act as a secondary detection after the optical tagging. Logos are shown for the various instutions at which each component is being developed. Reprinted from [43].

\subsubsection{The Adiabatic Approximation}

In order to understand the functionality of the ion trap, the potential created around these electrodes must be examined. While the linear quadrupole (LQ) is made of four cylinders, a true quadrupole uses hyperbolic shaped electrodes. Unfortunately hyperbolic electrodes are difficult to manufacture properly so cylinders with carefully chosen radii $\left(r_{\mathrm{e}}=1.148 r_{0}\right)$ are used as a close approximation (see figure 5.2 [19]).

A proper hyperbolic quadrupole produces a radial potential of the form:

$$
\Phi_{\mathrm{RF}}=\frac{1}{2} V \cos \Omega t\left(\frac{x^{2}-y^{2}}{r_{0}^{2}}\right)
$$

The gradient of equation 5.1 can then be calculated in order to obtain the electric field produced by the LQ:

$$
\vec{E}_{\mathrm{RF}}=-\nabla \Phi_{\mathrm{RF}}=-V \cos \Omega t\left(\frac{x \hat{x}-y \hat{y}}{r_{0}^{2}}\right)
$$

The motion of a charged particle can now be examined within this field using Newton's second law of motion, $F=m a$ and substituting in $F=Q E$. To start, a 


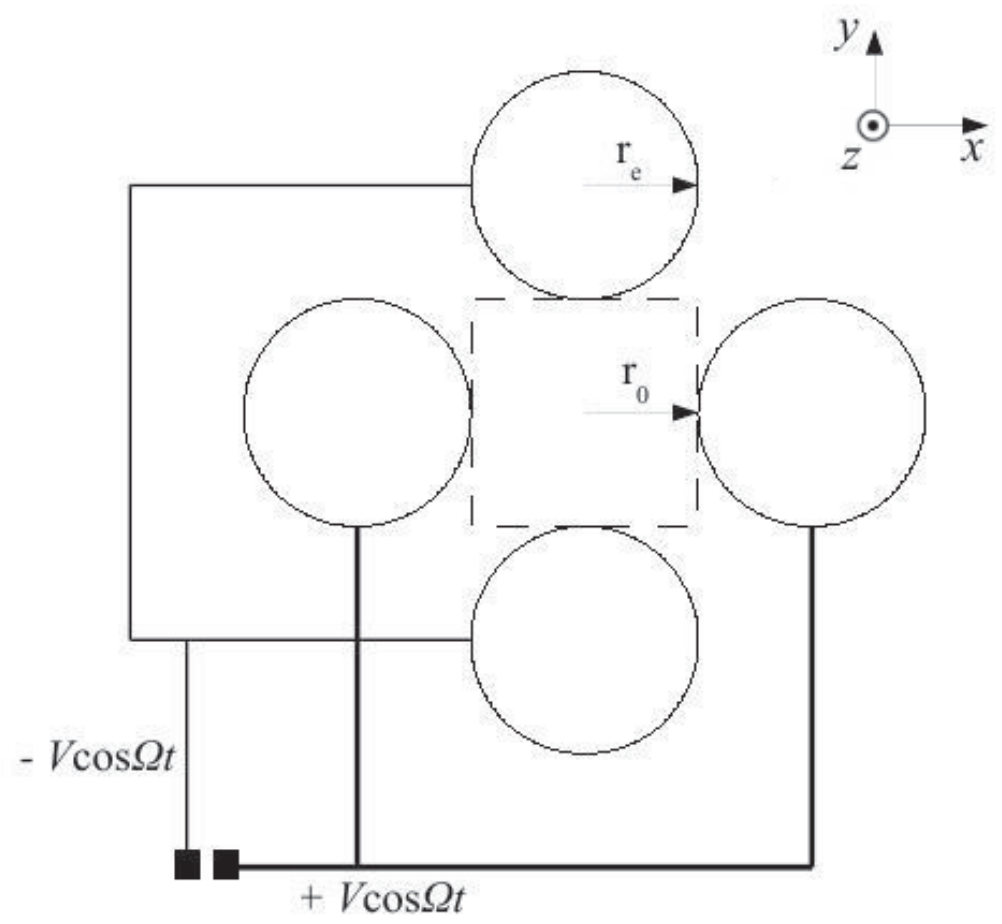

Figure 5.2: Cross-section of the electrode layout of a typical linear quadrupole. Neighbouring rods carry RF voltage of opposite sign. Adapted from [19].

generic oscillating electric field, $E_{0}(r) \cos \Omega t$, is considered which will later be replaced with the electric field from equation 5.2.

$$
m \ddot{r}(t)=Q E_{0}(r) \cos \Omega t
$$

If a homogeneous electric field is assumed, the trivial solution of harmonic motion about the origin is found,

$$
r(t)=-\frac{Q E_{0}}{m \Omega^{2}} \cos \Omega t
$$

This simple solution can be extended toward a slightly inhomogeneous field, in which case the particle is expected to follow some slowly oscillating main trajectory (macromotion) while exhibiting harmonic oscillation similar to before (micromotion), now centered on this new main trajectory. Here an approximation is made that the amplitude of the fast oscillation is negligible compared to the overall motion of the particle. This is the primary assumption of the adiabatic approximation. 


$$
r(t)=R_{0}(t)-\frac{Q}{m \Omega^{2}} E_{0}(t) \cos \Omega t
$$

Here $R_{0}(t)$ represents the new main trajectory and the rest of the equation is similar to the trivial solution.

By performing a Taylor series expansion of the electric field about $R_{0}$ and discarding all but the first two terms it is found that,

$$
E(r, t)=E_{0}\left(R_{0}\right) \cos \Omega t-\frac{Q}{m \Omega^{2}} E_{0} \frac{d E_{0}}{d r} \cos ^{2} \Omega t
$$

The previous two equations can be considered together by plugging them into the left and right sides of $m \ddot{r}(t)=Q E(t)$ respectively to find,

$$
m \ddot{R}_{t}+Q E_{0}(r) \cos \Omega t=Q\left[E_{0}\left(R_{0}\right) \cos \Omega t-\frac{Q}{m \Omega^{2}} E_{0} \frac{d E_{0}}{d r} \cos ^{2} \Omega t\right]
$$

Since it is still assumed the inhomogeneity of the field is small, the electric field can be treated as constant on the scale of the small oscillatory motion and $E(r)=E\left(R_{0}\right)$. The two cos $\Omega t$ terms now cancel. This can be further simplified by taking the time average of the remaining equation of motion to find,

$$
m \ddot{R}_{0}=-\frac{Q^{2}}{4 m \Omega^{2}} \frac{d}{d r}\left\langle E_{0}^{2}\right\rangle=-\nabla\left[\frac{Q^{2}\left\langle E_{0}^{2}\right\rangle}{4 m \Omega^{2}}\right]
$$

An effective potential can now be defined,

$$
\Phi_{\text {eff }}\left(R_{0}\right)=\frac{Q\left\langle E_{0}^{2}\left(R_{0}\right)\right\rangle}{4 m \Omega^{2}}
$$

This equation is now in a form which can be used to examine the behaviour of a particle in a quadrupole, however before doing so an important feature of this equation must be noted; the dependence on the gradient of the squared electric field. This dependence means the the particle is (over an extended time scale) always attracted towards regions of minimal electric field magnitude. This observation along with the form of the quadrupole electric field already indicates that the particle should be confined to the centre of the quadrupole without even solving the equation of motion. In order to see some of the more subtle details of the quadrupole potential however, this will be examined further.

The time average and square of the quadrupole electric field from equation 5.2 are now computed, 


$$
\left\langle E_{\mathrm{RF}}^{2}\right\rangle=\left\langle\vec{E}_{\mathrm{RF}} \cdot \vec{E}_{\mathrm{RF}}\right\rangle=\frac{V^{2}}{2} \frac{r^{2}}{r_{0}^{4}}
$$

When this is substituted into equation 5.9 the result is a simple harmonic oscillator potential,

$$
\Phi_{e f f}=\frac{Q V^{2}}{8 m \Omega^{2} r_{0}^{4}} r^{2}
$$

This potential form is what will be used to confine ions axially within the ion trap.

\subsubsection{Linear Quadrupole Mass Filters}

The analysis of the quadrupole potential can be taken one step further by examining the case of a quadrupole in which the electrodes carry a DC voltage as well as the RF voltage. Referring back to figure 5.2, now the potential is modified, $\pm V \cos \Omega t \rightarrow \pm(U+V \cos \Omega t)$. It will be seen that the addition of a DC voltage allows the quadrupole to act as a mass filter.

The electric field can be broken down separately into $\mathrm{x}$ and $\mathrm{y}$ components to more easily analyze the behavior. It will now have the form,

$$
E_{u}=\mp(U+V \cos \Omega t) \frac{u}{r_{0}^{2}}
$$

where $u \in\{\mathrm{x}, \mathrm{y}\}$ and the sign in front is negative for $\mathrm{x}$, positive for $\mathrm{y}$. Now the equations of motion will be,

$$
m \ddot{u}(t)=\mp \frac{Q}{r_{0}^{2}}[U+V \cos \Omega t] u
$$

where again the sign is negative for $u=\mathrm{x}$ and positive for $u=\mathrm{y}$. A few variables can now be defined that will allow equation 5.13 to be rewritten,

$$
\begin{aligned}
\tau & =\frac{1}{2} \Omega t \\
a_{\mathrm{u}} & = \pm \frac{4 Q U}{m \Omega^{2} r_{0}^{2}} \\
q_{\mathrm{u}} & =\mp \frac{2 Q V}{m \Omega^{2} r_{0}^{2}}
\end{aligned}
$$




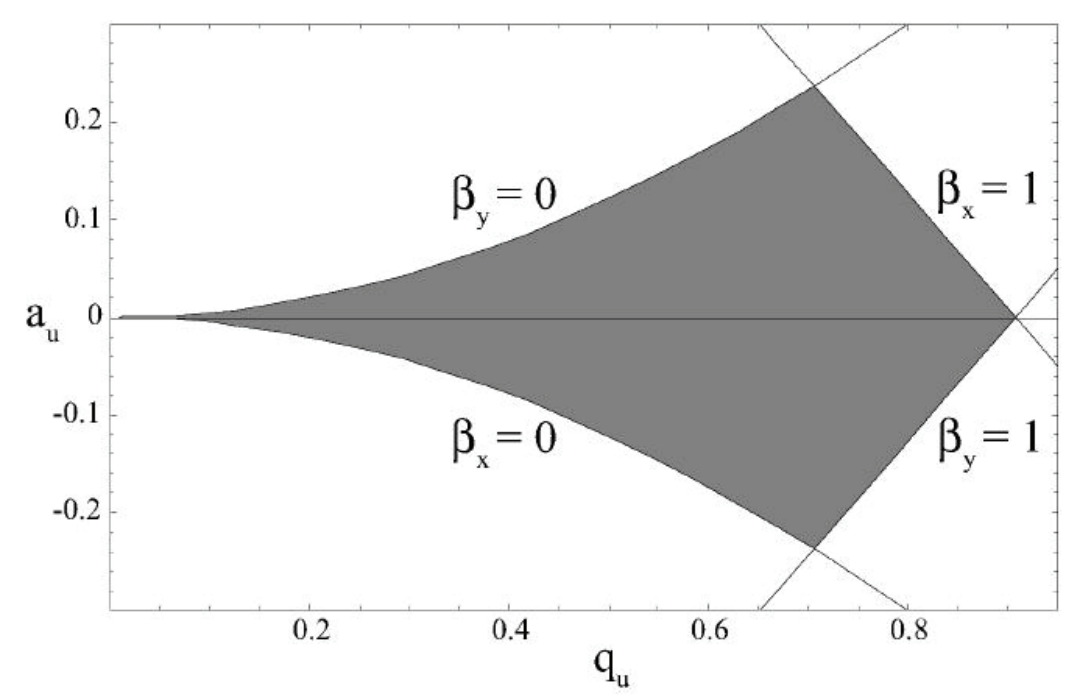

Figure 5.3: Region of stable ion trajectory bounded by $\beta_{\mathrm{u}}=0,1$. Reprinted from $[13]$.

Now equation 5.13 becomes,

$$
\ddot{u}(\tau)+\left[a_{\mathrm{u}}-2 q_{\mathrm{u}} \cos 2 \tau\right] u=0
$$

which is the general form of the homogenous Mathieu equation. The Mathieu equation is a well-studied differential equation with known series solutions of the form,

$$
u(\tau)=A e^{i \beta_{\mathrm{u}} \tau} \sum_{n=-\infty}^{\infty} C_{2 n} e^{i 2 n \tau}+B e^{-i \beta_{\mathrm{u}} \tau} \sum_{n=-\infty}^{\infty} C_{2 n} e^{-i 2 n \tau}
$$

In this solution, $A$ and $B$ are constants chosen to satisfy the initial conditions of the particle and the coefficients $C_{2 n}$ and $\beta_{\mathrm{u}}$ depend only on $a_{\mathrm{u}}$ and $q_{\mathrm{u}}$. For the particle trajectory to be stable (i.e. the ion path does not diverge) it is required that $\beta_{\mathrm{u}}$ be a real, non-integer number. For small $a_{\mathrm{u}}$ and $q_{\mathrm{u}}, \beta_{\mathrm{u}}$ can be approximated to $\beta_{\mathrm{u}}= \pm \sqrt{\frac{1}{2} q_{\mathrm{u}}^{2}+a_{\mathrm{u}}}[19]$ and a stability region can be defined in $a_{\mathrm{u}}, q_{\mathrm{u}}$ space bounded by $\beta_{\mathrm{u}} \in(0,1)$ as shown in figure 5.3 .

At this point it can be observed that the parameters $a_{\mathrm{u}}$ and $q_{\mathrm{u}}$ depend only on characteristics of the quadrupole $\left(U, V, \Omega, r_{0}\right)$ and of the particle $\left(\frac{Q}{m}\right)$. It then follows that for a given set of quadrupole parameters only particles within a particular range of charge-to-mass ratio will have a stable trajectory. This principle is what allows the design of a quadrupole mass filter. This concludes all of the physics knowledge 
required to contruct a linear quadrupole ion trap and or mass filter.

\subsubsection{The Second Generation Stanford Linear Quadrupole Ion Trap}

The ion trap in use at Carleton is the second generation trap constructed at Stanford University and explained in detail in [13]. The basic geometry of the trap is a set of stainless steel cylindrical electrodes, similar to those shown in figure 5.2, now with $r_{\mathrm{e}}=3 \mathrm{~mm}$ and $r_{0}=2.66 \mathrm{~mm}$.

\section{Trap Structure}

In order to create a longitudinal potential well, the ion trap is divided into 16 shorter quadrupole segments each of which has a DC potential in addition to the normal quadrupole RF potential. The potential on each rod now has the form $V_{D C} \pm V \cos \Omega t$ (not to be confused with the mass filter potential which had the form $\pm(U+V \cos \Omega t))$. These $\mathrm{DC}$ potentials do not affect the radial confinement of the ions since they are approximately uniform in $\mathrm{x}$ and $\mathrm{y}$ and can be configured to create a longitudinal potential well. In the set up, segments 13 and 14 are designated as the trapping segments (segment 1 being the trap entrance and segment 16 the trap exit) and therefore the lowest DC potential is set here. Each trap segment is $50 \mathrm{~mm}$ long (49.5 mm electrodes with $0.5 \mathrm{~mm}$ spacing) with the exception of the two trapping segments which are each $5 \mathrm{~mm}$ (4 mm electrodes with $1 \mathrm{~mm}$ spacing). This gives a total trap length of $71 \mathrm{~cm}$. The longitudinal potential created by this segmented trap design is shown in figure 5.4. In this configuration a sharp potential well has been created at the trapping segments (13 and 14) and a tall potential barrier has been created at segment 16 to prevent ions from flying all the way through the trap.

The trap electrodes are mounted in pairs onto macor blocks which provide electrical isolation and a rigid frame to maintain proper alignment (each macor block supports two rods with the exception of the trapping segment blocks which each support four shorter rods). These blocks are then mounted on a stainless steel frame with push pins to help with alignment. The entire assembly can be seen in figure 5.5.

The assembled trap structure is mounted inside a vacuum chamber for operation. The vacuum within the chamber is maintained using an Edwards nEXT400 turbomolecular pump [44] and monitored using two Pfeiffer PKR 261 full range Pirani/cold 

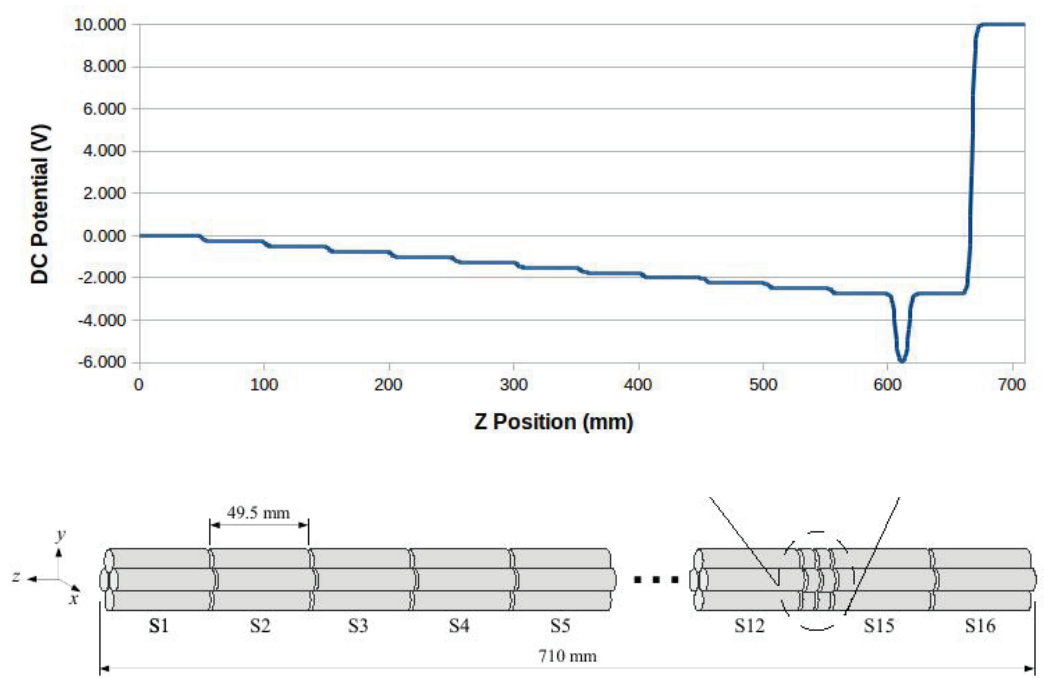

Figure 5.4: The longitudinal potential is shown along the centre $(r=0 \mathrm{~mm}$ of the trap using DC offset potentials found in table 5.1. This potential was calculated using the SIMION ion flight simulation software. Below, the segmentation of the ion trap is illustrated. Adapted from [19].

cathode pressure gauges [45]. When baked out, the pressure in the vacuum chamber typically ranges from $10^{-} 9$ to $10^{-} 8$ mbar.

\section{Electronics}

To provide the voltage on each trapping segment a number of different power supplies are used. A small amplitude RF signal is created using a BK Precision 4005DDS function generator [46] which is then amplified using a custom made RF amplifier powered by an HP 6227B DC power supply [47]. This RF potential is then mixed with the 16 different DC offset voltages (provided by an Extrel MS Command System 20-pin analog output [48]) and passed through a set of 10-pin feedthrough ports and then connected to their corresponding electrodes (diagonally opposite electrodes are wired together to simplify connections). An RF/DC mixing circuit is shown in figure 5.6. Sixteen identical circuits were used in total. The assembled trap can be seen in figure 5.7 . 


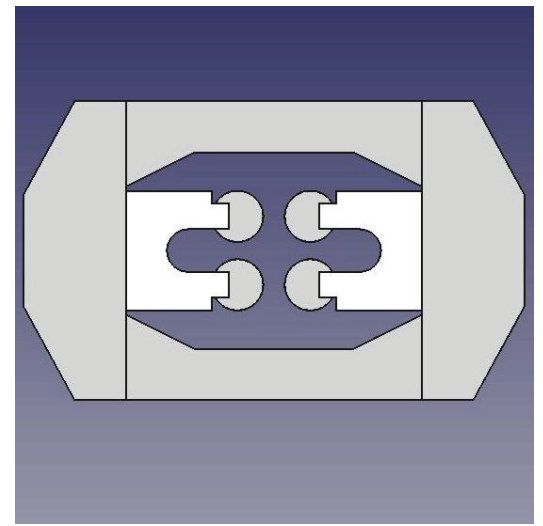

(a) Trap entrance

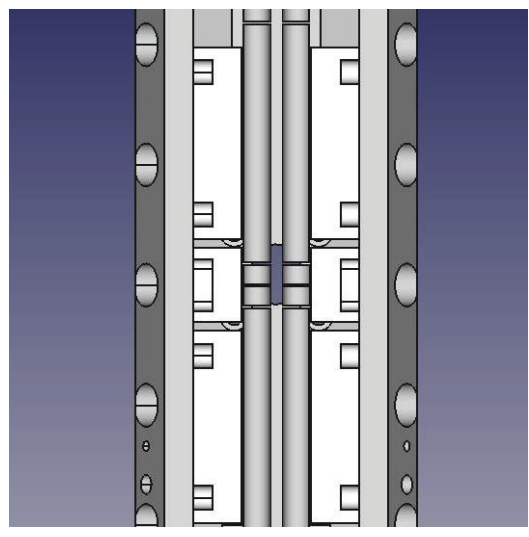

(b) Trapping region

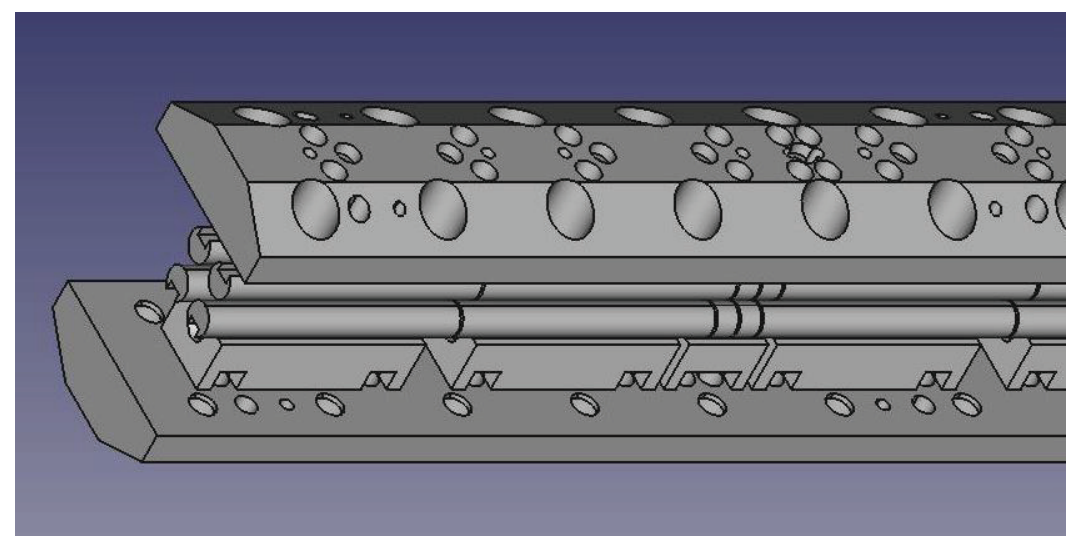

(c) Trap assembly

Figure 5.5: A CAD model of the ion trap is shown here depicting the trap electrodes, macor mounting block and steel support frace. Figure 5.5(a) shows the front face of the ion trap. Figure 5.5(b) shows the ion trap from the top, focusing on the trapping region. Figure 5.5(c) shows an isometric view of the ion trap assembly. 


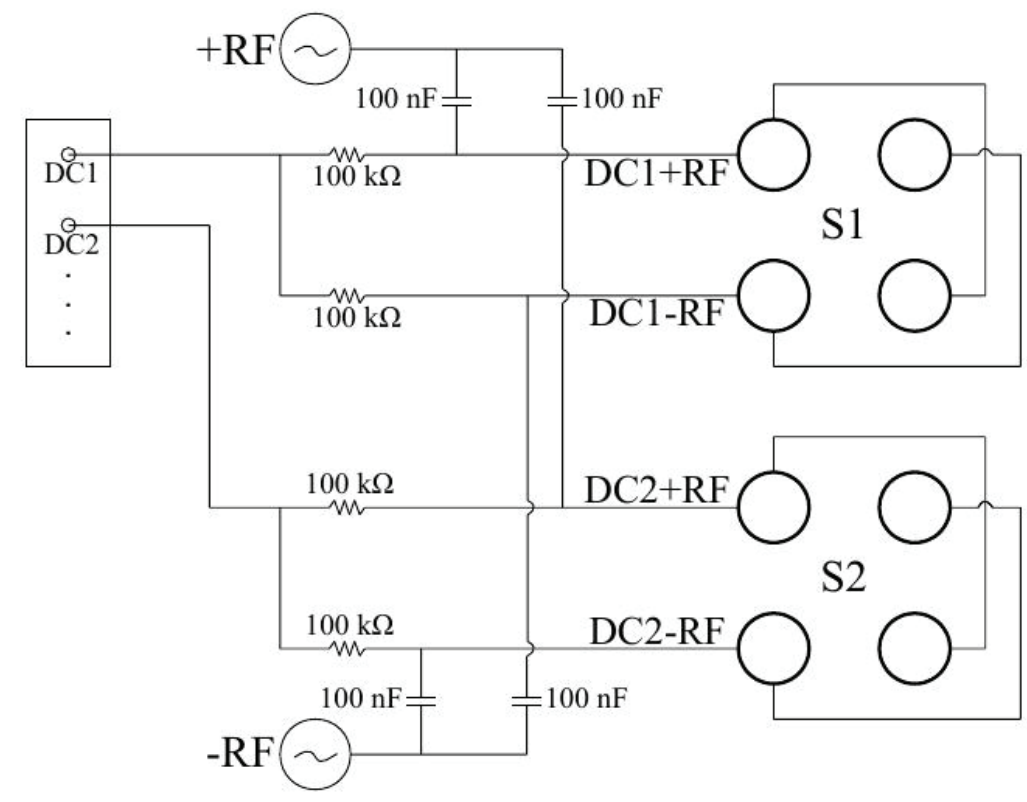

Figure 5.6: A single RF/DC mixing circuit used combine the quadrupole RF potentials with the individual segment DC offset voltages. Reprinted from [19].

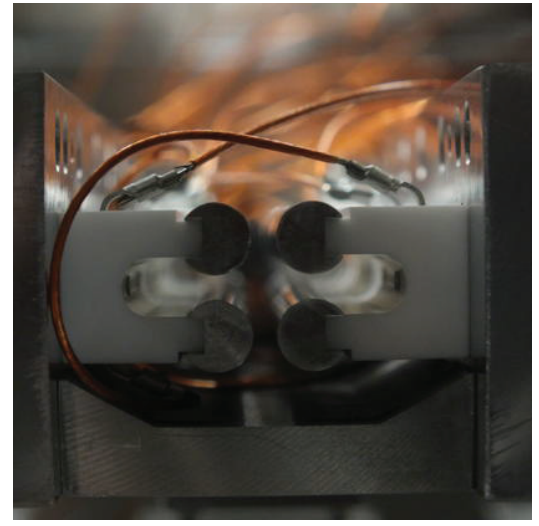

(a) Trap entrance

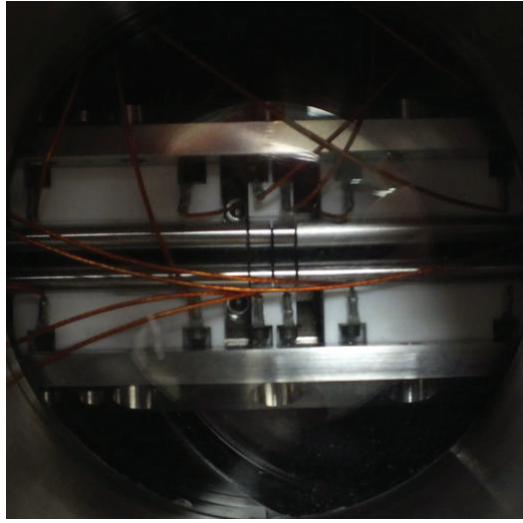

(b) Trap entrance

Figure 5.7: Some photos of the assembled ion trap. Figure 5.7(a) shows the entrance of the trap. Figure 5.7(b) shows the trapping region as seen through one of the vacuum chamber viewports. Some of the wiring is visible in both photos which carries the mixed DC and RF voltages to the rods. 


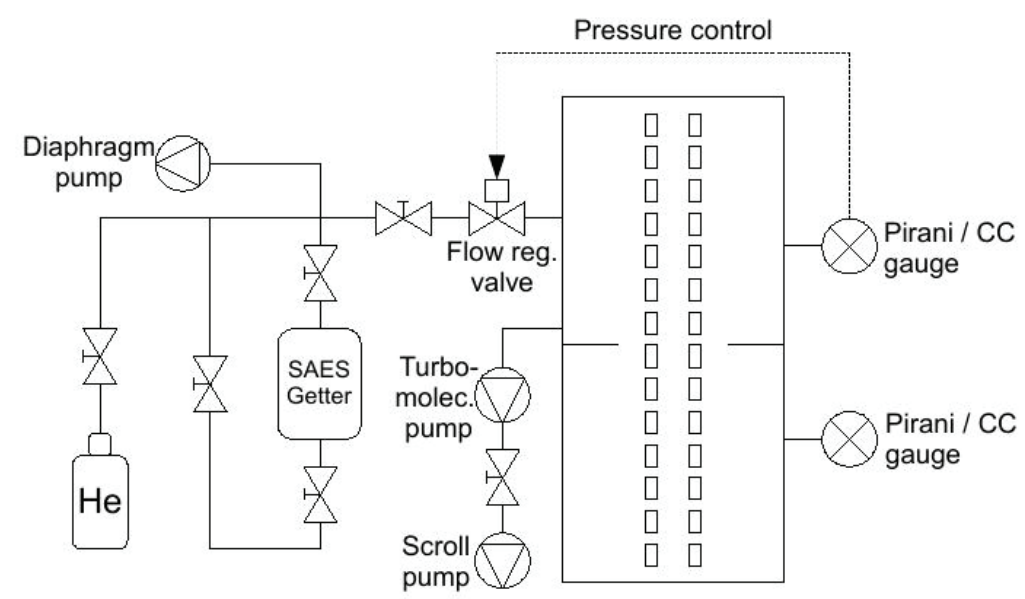

Figure 5.8: A schematic of the buffer gas supply system to the ion trap. Figure from [19].

\section{Ion Cooling with an Inert Buffer Gas}

A practical consideration which must be made is that in order to become trapped, an ion must lose energy as it travels through the trap, otherwise (in accordance with conservation of energy) it will either fly out the other end of the trap or be reflected back out through the entrance. To cool the ion (i.e. to reduce it's energy) an inert buffer gas is introduced into the system. A few different gasses for this purpose have been studied in detail in [13] and [19] and it was found that either helium or argon are suitable for ion cooling. In this case helium is used which is introduced into the system through a Pfeiffer UDV 146 needle valve [49] which allows very precise control over the buffer gas pressure. When setting the buffer gas pressure, it is assumed that the background gas pressure is several orders of magnitude less than the buffer gas pressure and therefore the total system pressure is approximately equal to the buffer gas pressure. Additionally, a SAES Getter [50] is used to remove impurities in the supply line in order to keep a clean vacuum. The buffer gas supply system is illustrated in figure 5.8 [19].

\section{Operating Parameters}

Most system parameters for successful operation of the ion trap have been standardized and are presented in table 5.1. The trap is less sensitive to the buffer gas pressure and therefore a range of pressures is presented within which the trap has been shown 
to perform well.

\subsection{Optical Tagging}

Once a barium ion has been trapped some technique must be used to detect it. For the purposes of $\mathrm{nEXO}$, there are a couple requirements:

1. The technique must be able to differentiate barium from other ions.

2. The technique should be sensitive to the single ion level.

To meet the first requirement a two laser system is used to induce fluorescence in the trapped ions and the emitted light is then detected using a photomultiplier tube (PMT). It is due to some interesting properties of the three level Ba-II system (the valence level and closest empty energy levels of the $\mathrm{Ba}^{+}$ion) that this technique can be used to tell barium apart from other ions. This topic will be explored in more depth shortly. With the first requirement fullfilled, the second is simply a matter of carefully controlling lab conditions and properly tuning the detection setup to achieve a strong enough sensitivity. The conditions and tuning that allow for this sensitivity will discussed later.

\subsubsection{The Ba-II System}

The $\mathrm{Ba}^{+}$ion has a single electron in its $6 \mathrm{~s}$ valence shell. This shell along with the two closest neighbors, the $6 \mathrm{p}$ and $5 \mathrm{~d}$ shells form what is known as the Ba-II system. In this 3-level system there are only two allowed dipole transitions, one between the $6 \mathrm{~s}$ (the ground state) and 6p (first excited state) levels, the other between the 6p and $5 \mathrm{~d}$ (meta-stable state) levels. Because there is no allowed dipole transition between the $6 \mathrm{~s}$ and $5 \mathrm{~d}$ states, a $\mathrm{Ba}^{+}$ion which is excited to the $5 \mathrm{~d}$ state cannot easily decay back to its ground state. There is a quadrupole transition which allows the transition

from $5 \mathrm{~d} \rightarrow 6 \mathrm{~s}$ however this decay has such a long lifetime compared to the dipole transitions ( $\sim 10$ orders of magnitude) that the $5 \mathrm{~d}$ state is termed meta-stable. The allowed dipole transitions of the Ba-II system are illustrated in figure 5.9.

It is the meta-stable nature of the $5 \mathrm{~d}$ state that can be exploited to detect and identify the $\mathrm{Ba}^{+}$ions. By continually pumping the $6 \mathrm{~s} \rightarrow 6 \mathrm{p}$ transition (typically done with a $493 \mathrm{~nm}$ laser) any ions in the trap are forced into the meta-stable $5 \mathrm{~d}$ state. 
Table 5.1: Operating parameters for the second generation ion trap.

\begin{tabular}{|c|c|c|}
\hline Parameter name & Description & Value \\
\hline $\begin{array}{c}P \\
\Omega \\
V_{\mathrm{PP}} \\
V_{\mathrm{DC}}\end{array}$ & $\begin{array}{l}\text { Buffer gas pressure. } \\
\text { The frequency of the RF voltage. } \\
\text { The peak-to-peak RF voltage am- } \\
\text { plitude }\left(V_{\mathrm{PP}}=2 \mathrm{~V}\right) \text {. } \\
\text { The DC offset voltage of each trap } \\
\text { segment. Voltages are presented } \\
\text { in order from segment } 1 \text { to seg- } \\
\text { ment } 16 \text {. }\end{array}$ & $\begin{array}{c}5 \times 10^{-5}-5 \times 10^{-3} \text { mbar } \\
1.45 \mathrm{MHz} \\
200 \mathrm{~V} \\
0.00 \mathrm{~V} \\
-0.25 \mathrm{~V} \\
-0.50 \mathrm{~V} \\
-0.75 \mathrm{~V} \\
-1.00 \mathrm{~V} \\
-1.25 \mathrm{~V} \\
-1.50 \mathrm{~V} \\
-1.75 \mathrm{~V} \\
-2.00 \mathrm{~V} \\
-2.25 \mathrm{~V} \\
-2.50 \mathrm{~V} \\
-2.75 \mathrm{~V} \\
-6.00 \mathrm{~V}, \text { trapping region } \\
-2.75 \mathrm{~V} \\
10.00 \mathrm{~V} \\
\text { trapping region } \\
-6.00 \mathrm{~V}\end{array}$ \\
\hline
\end{tabular}




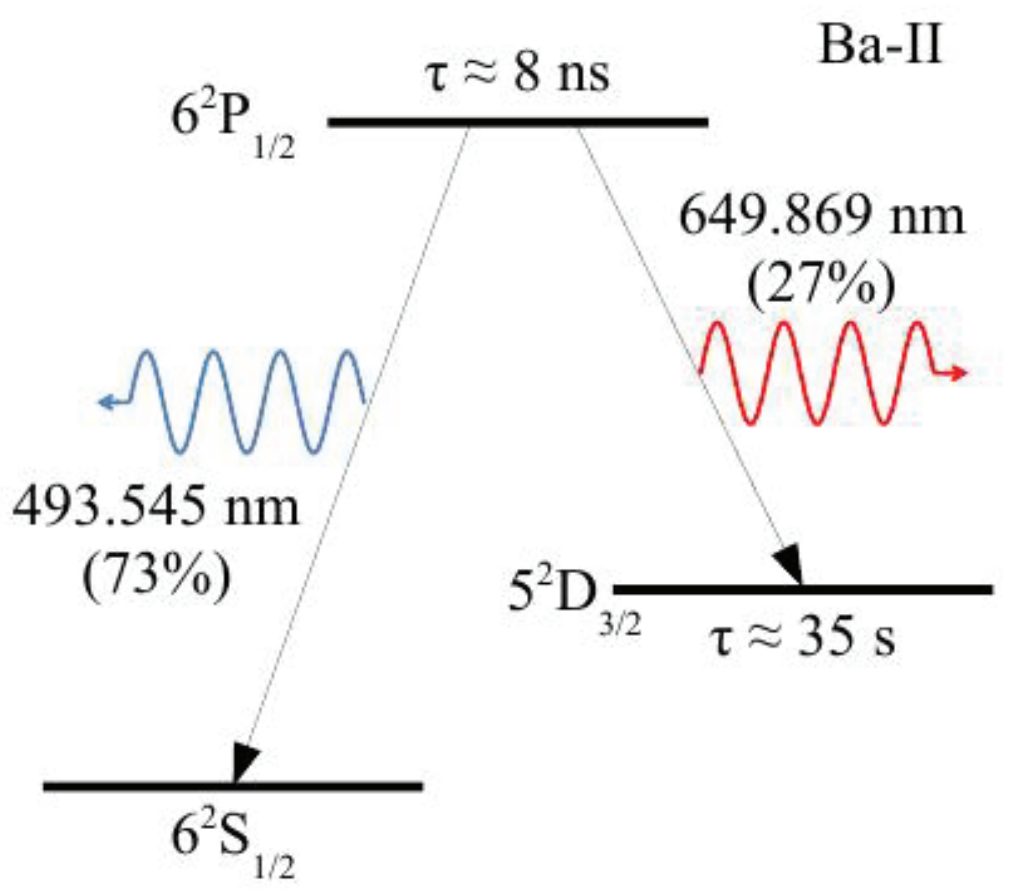

Figure 5.9: The three energy levels of the Ba-II system with lifetimes and branching ratios labelled as well as the wavelengths of dipole transition photons. Figure from [19].

When excited by the laser into the $6 \mathrm{p}$ state, the ions can decay to either the ground state or the meta-stable state. A decay back to ground state releases a photon at 493 $\mathrm{nm}$ which can be detected, and allows the pumping to continue, however if the ion decays to the meta-stable state it releases a $649 \mathrm{~nm}$ photon and the pumping stops. Because of the short lifetime of the $6 \mathrm{p}$ state and relatively long lifetime of the $5 \mathrm{~d}$ state, when an ion is stimulated continuously with $493 \mathrm{~nm}$ laser light it very quickly becomes trapped in the $5 \mathrm{~d}$ state and no more fluorescence is detected. If the ion is then stimulated with a $649 \mathrm{~nm}$ laser to pump the $5 \mathrm{~d} \rightarrow 6 \mathrm{p}$ transition the fluorescence will begin again. If the amount of fluorescence light detected with only the $493 \mathrm{~nm}$ laser is compared to the amount detected with both a $493 \mathrm{~nm}$ and a $649 \mathrm{~nm}$ laser, the difference will be entirely fluoresence from the $6 \mathrm{p} \rightarrow 6$ s transition and from the magnitude of this difference the number of $\mathrm{Ba}^{+}$ions present within the trap can be determined. 


\subsubsection{The Optical Bloch Equations}

To understand what is being observed with this technique, the behaviour of this 3level quantum system when exposed to two laser fields must be fully examined. To do this, the density matrix formalism will be utilized. This formalism is explored more fully in [19]. To begin, the three quantum states of the system can be represented by,

$$
\begin{aligned}
& |a\rangle=\left|6^{2} \mathrm{P}_{1 / 2}\right\rangle \\
& |b\rangle=\left|6^{2} \mathrm{~S}_{1 / 2}\right\rangle \\
& |c\rangle=\left|5^{2} \mathrm{D}_{3 / 2}\right\rangle
\end{aligned}
$$

and let $\omega_{\mathrm{B}, \mathrm{R}}$ represent the frequencies of the lasers. The subscripts $\mathrm{B}$ and $\mathrm{R}$ are chosen due to the colors of the lasers on the visible spectrum $(\mathrm{B}=$ blue $=493 \mathrm{~nm}, \mathrm{R}=\mathrm{red}=$ $649 \mathrm{~nm})$. Furthermore, the energy of each eigenstate can be written $\hbar \omega_{a, b, c}$. Now the Hamiltonian of the 3-level system can be written. Beginning with the unperturbed system (i.e. the 3 -level system with no outside fields present), $H_{0}$,

$$
H_{0}|j\rangle=\hbar \omega_{j}|j\rangle
$$

where $j \in\{a, b, c\}$.

If the laser electric fields are represented as $\vec{E}_{\mathrm{B}, \mathrm{R}}$ and the dipole operator for transition between two quantum state $(|m\rangle$ and $|n\rangle)$ is represented as $\vec{d}=$ $\vec{d}_{n m}(|n\rangle\langle m|+| m\rangle\langle n|)$ (where $\vec{d}_{n m}$ is the dipole matrix element), then the interation Hamiltonian from each laser field can be written as,

$$
H_{\mathrm{B}, \mathrm{R}}=-\vec{d} \cdot \vec{E}_{\mathrm{B}, \mathrm{R}} \cos \omega_{\mathrm{B}, \mathrm{R}} t
$$

The Hamiltonians, $H_{\mathrm{B}, \mathrm{R}}$ can now be rewritten in terms of the raising and lowering operators $(|n\rangle\langle m|$ and $|m\rangle\langle n|)$ and the cosine terms can be expanded into exponential form,

$$
\begin{aligned}
& H_{\mathrm{B}}=-\frac{1}{2}\langle b|\vec{d}| a\rangle \cdot \vec{E}_{\mathrm{B}}\left[|a\rangle\left\langle b\left|\left(e^{i \omega_{\mathrm{B}} t}+e^{-i \omega_{\mathrm{B}} t}\right)+\right| b\right\rangle\langle a|\left(e^{i \omega_{\mathrm{B}} t}+e^{-i \omega_{\mathrm{B}} t}\right)\right] \\
& H_{\mathrm{R}}=-\frac{1}{2}\langle c|\vec{d}| a\rangle \cdot \vec{E}_{\mathrm{R}}\left[|a\rangle\left\langle c\left|\left(e^{i \omega_{\mathrm{R}} t}+e^{-i \omega_{\mathrm{R}} t}\right)+\right| c\right\rangle\langle a|\left(e^{i \omega_{\mathrm{R}} t}+e^{-\imath \omega_{\mathrm{R}} t}\right)\right]
\end{aligned}
$$


In both Hamiltonians each exponential term can now be interpreted differently. Exponentials with a $i \omega_{\mathrm{B}, \mathrm{R}} t$ represent the emission of a photon whereas those with a $-i \omega_{\mathrm{B}, \mathrm{R}} t$ represent the absorption of a photon. If the raising/lowering operators are inspected, it is clear which transition each exponential corresponds to.

If the rotating wave approximation (RWA) [51] is used, terms in which the energy state is raised and a photon is released as well as terms in which the energy state is lowered and a photon is absorbed can be eliminated. The Hamiltonians can also be tidied up a little by defining the Rabi frequencies $\hbar \Omega_{\mathrm{B}}=-\langle b|\vec{d}| a\rangle \cdot \vec{E}_{\mathrm{B}}$ and $\hbar \Omega_{\mathrm{R}}=-\langle c|\vec{d}| a\rangle \cdot \vec{E}_{\mathrm{R}}$. The simplified Hamiltonians now have the form,

$$
\begin{aligned}
& H_{\mathrm{B}}^{\mathrm{RWA}}=\frac{1}{2} \hbar \Omega_{\mathrm{B}}\left(|a\rangle\left\langle b\left|e^{i \omega_{\mathrm{B}} t}+\right| b\right\rangle\langle a| e^{-i \omega_{\mathrm{B}} t}\right) \\
& H_{\mathrm{R}}^{\mathrm{RWA}}=\frac{1}{2} \hbar \Omega_{\mathrm{R}}\left(|a\rangle\left\langle c\left|e^{i \omega_{\mathrm{R}} t}+\right| c\right\rangle\langle a| e^{-i \omega_{\mathrm{R}} t}\right)
\end{aligned}
$$

The full Hamiltonian of the system $\left(H_{\mathrm{RWA}}=H_{0}+H_{\mathrm{B}}^{\mathrm{RWA}}+H_{\mathrm{R}}^{\mathrm{RWA}}\right)$ can now be rewritten in matrix form.

$$
H_{R W A}=\left[\begin{array}{ccc}
\hbar \omega_{a} & \frac{1}{2} \hbar \Omega_{B} e^{-i \omega_{B} t} & \frac{1}{2} \hbar \Omega_{R} e^{-i \omega_{R} t} \\
\frac{1}{2} \hbar \Omega_{B} e^{i \omega_{B} t} & \hbar \omega_{b} & 0 \\
\frac{1}{2} \hbar \Omega_{R} e^{i \omega_{R} t} & 0 & \hbar \omega_{c}
\end{array}\right]
$$

The effects of this Hamiltonian on the 3-level quantum system can now be explored. To do this the density matrix, $\rho$, which describes the population densities of the three states as well as the coherences between each state will be examined,

$$
\rho=\left[\begin{array}{ccc}
\rho_{a a} & \rho_{a b} & \rho_{a c} \\
\rho_{b a} & \rho_{b b} & \rho_{b c} \\
\rho_{c a} & \rho_{c b} & \rho_{c c}
\end{array}\right]
$$

where the diagonal elements represent the population densities of the three quantum states and the off-diagonal elements are the coherences between these states.

In order to examine these effects, the Liouville equation which describes the time evolution of a population density under the influence of a given Hamiltonian will be used, 


$$
\frac{d \rho}{d t}=-\frac{i}{\hbar}[\mathcal{H}, \rho]+\gamma \rho
$$

where $\gamma$ is called the relaxation matrix and in this case represents the spontaneous emission between energy states. Unfortunately, the Liouville equation requires a time-independent Hamiltonian and so a change of basis must be made,

$$
\begin{aligned}
\mathcal{H}_{\mathrm{RWA}} & \rightarrow T \mathcal{H}_{\mathrm{RWA}} T^{\dagger}-i \hbar T \frac{d}{d t} T^{\dagger} \\
\rho & \rightarrow T \rho T^{\dagger}
\end{aligned}
$$

with the transformation matrix, T, given by,

$$
T=\left[\begin{array}{ccc}
1 & 0 & 0 \\
0 & e^{-i \omega_{\mathrm{B}} t} & 0 \\
0 & 0 & e^{-i \omega_{\mathrm{R}} t}
\end{array}\right]
$$

Using these transformations, the Hamiltonian and density matrices now take on new forms,

$$
\tilde{\mathcal{H}}_{\mathrm{RWA}}=\frac{\hbar}{2}\left[\begin{array}{ccc}
0 & \Omega_{\mathrm{B}} & \Omega_{\mathrm{R}} \\
\Omega_{\mathrm{B}} & 2 \delta_{\mathrm{B}} & 0 \\
\Omega_{\mathrm{R}} & 0 & 2 \delta_{\mathrm{R}}
\end{array}\right]
$$

and,

$$
\tilde{\rho}=\left[\begin{array}{ccc}
\rho_{a a} & \tilde{\rho}_{a b} & \tilde{\rho}_{a c} \\
\tilde{\rho}_{b a} & \rho_{b b} & \tilde{\rho}_{b c} \\
\tilde{\rho}_{c a} & \tilde{\rho}_{c b} & \rho_{c c}
\end{array}\right]
$$

Here several new quantities have been introduced in order to simplify the presentation of the transformed matrices. The $\delta_{\mathrm{B}, \mathrm{R}}$ represent the detuning of the laser fields from their relevant transition frequencies, 


$$
\delta_{\mathrm{B}, \mathrm{R}}=\omega_{\mathrm{B}, \mathrm{R}}-\left(\omega_{a}-\omega_{b, c}\right)
$$

and the $\tilde{\rho}_{i j}$ are the "slow" coherences,

$$
\begin{aligned}
& \tilde{\rho}_{a b}=\tilde{\rho}_{b a}^{*}=\rho_{a b} e^{i \omega_{\mathrm{B}} t} \\
& \tilde{\rho}_{a c}=\tilde{\rho}_{c a}^{*}=\rho_{a c} e^{i \omega_{\mathrm{R}} t} \\
& \tilde{\rho}_{b c}=\tilde{\rho}_{c b}^{*}=\rho_{b c} e^{i\left(\omega_{\mathrm{R}}-\omega_{\mathrm{B}}\right) t}
\end{aligned}
$$

The relaxation matrix, $\gamma$ can now be applied to $\tilde{\rho}$ to obtain the last piece required for use of the Liouville equation.

$$
\gamma \tilde{\rho}=\left[\begin{array}{ccc}
-\Gamma \rho_{a a} & -\frac{1}{2} \Gamma \tilde{\rho}_{a b} & -\frac{1}{2} \Gamma \tilde{\rho}_{a c} \\
-\frac{1}{2} \Gamma \tilde{\rho}_{b a} & \Gamma_{\mathrm{B}} \rho_{b b} & 0 \\
-\frac{1}{2} \Gamma \tilde{\rho}_{c a} & 0 & \Gamma_{\mathrm{R}} \rho_{c c}
\end{array}\right]
$$

where $\Gamma_{\mathrm{B}, \mathrm{R}}$ are the spontaneous decay rates from the $6 \mathrm{p}$ to $6 \mathrm{~s}$ and $5 \mathrm{~d}$ states respectively and $\Gamma=\Gamma_{B}+\Gamma_{R}$ is the total spontaneous decay rate of the $6 \mathrm{p}$ state.

Now applying the Liouville equation gives nine differential equations, known as the Optical Bloch Equations (OBEs), one for each density matrix element. It can reasonably be approximated that the lasers will be on much longer than the lifetime of the $6 \mathrm{p}$ state and therefore only steady state solutions are required (i.e. set $\frac{d \tilde{\rho}}{d t}=0$ ). This gives a set of simplified equations for the diagonal elements,

$$
\begin{aligned}
\Gamma \rho_{a a} & =\frac{i}{2}\left[\Omega_{\mathrm{B}}\left(\tilde{\rho}_{a b}-\tilde{\rho}_{b a}\right)+\Omega_{\mathrm{R}}\left(\tilde{\rho}_{a c}-\tilde{\rho}_{c a}\right)\right] \\
\Gamma_{\mathrm{B}} \rho_{a a} & =\frac{i}{2} \Omega_{\mathrm{B}}\left(\tilde{\rho}_{a b}-\tilde{\rho}_{b a}\right) \\
\Gamma_{\mathrm{R}} \rho_{a a} & =\frac{i}{2} \Omega_{\mathrm{R}}\left(\tilde{\rho}_{a c}-\tilde{\rho}_{c a}\right)
\end{aligned}
$$

and for the off-diagonal elements (ignoring half of them due to symmetry in the matrix), 


$$
\begin{aligned}
\Gamma \tilde{\rho}_{a b} & =i\left[\Omega_{\mathrm{B}}\left(\rho_{a a}-\rho_{b b}\right)-\Omega_{\mathrm{R}} \tilde{\rho}_{c b}+2 \delta_{\mathrm{B}} \tilde{\rho}_{a b}\right] \\
\Gamma \tilde{\rho}_{a c} & =i\left[\Omega_{\mathrm{R}}\left(\rho_{a a}-\rho_{c c}\right)-\Omega_{\mathrm{B}} \tilde{\rho}_{b c}+2 \delta_{\mathrm{R}} \tilde{\rho}_{a c}\right] \\
0 & =\frac{i}{2}\left[\Omega_{\mathrm{R}} \tilde{\rho}_{b a}-\Omega_{\mathrm{B}} \tilde{\rho}_{a c}+2\left(\delta_{\mathrm{B}}-\delta_{\mathrm{R}}\right) \tilde{\rho}_{b c}\right]
\end{aligned}
$$

Solutions to this set of equations have already been derived in [52]. Here the solution for the excited state population density, $\rho_{a a}$, is shown since this of greatest interest in this experiment.

$$
\rho_{a a}=\frac{4}{Z}\left(\delta_{\mathrm{B}}-\delta_{\mathrm{R}}\right)^{2} \Omega_{\mathrm{B}}^{2} \Omega_{\mathrm{R}}^{2} \Gamma
$$

where,

$$
\begin{aligned}
Z= & 8\left(\delta_{\mathrm{B}}-\delta_{\mathrm{R}}\right)^{2} \Omega_{\mathrm{B}}^{2} \Omega_{\mathrm{R}}^{2} \Gamma+4\left(\delta_{\mathrm{B}}-\delta_{\mathrm{R}}\right)^{2} \Gamma^{2} Y \\
& +16\left(\delta_{\mathrm{B}}-\delta_{\mathrm{R}}\right)^{2}\left(\delta_{\mathrm{B}}^{2} \Omega_{\mathrm{R}}^{2} \Gamma_{\mathrm{B}}+\delta_{\mathrm{R}}^{2} \Omega_{\mathrm{B}}^{2} \Gamma_{\mathrm{R}}\right) \\
& -8 \delta_{\mathrm{B}}\left(\delta_{\mathrm{B}}-\delta_{\mathrm{R}}\right) \Omega_{\mathrm{R}}^{4} \Gamma_{\mathrm{B}}+8 \delta_{\mathrm{R}}\left(\delta_{\mathrm{B}}-\delta_{\mathrm{R}}\right) \Omega_{\mathrm{B}}^{4} \Gamma_{\mathrm{R}} \\
& +\left(\Omega_{\mathrm{B}}^{2}+\Omega_{\mathrm{R}}^{2}\right)^{2} Y
\end{aligned}
$$

and,

$$
Y=\Omega_{\mathrm{B}}^{2} \Gamma_{\mathrm{R}}+\Omega_{\mathrm{R}}^{2} \Gamma_{\mathrm{B}}
$$

From the excited state population the total fluorescence rate (i.e. the signal) can easily be calculated by multiplying the excited state population by the spontaneous decay rate to either the ground or meta-stable state (i.e. Photon $\operatorname{Rate}_{\mathrm{B}, \mathrm{R}}=\Gamma_{\mathrm{B}, \mathrm{R}} \rho_{a a}$ ). Note that this is the photon rate before accounting for detection efficiency and other experimental considerations.

\subsection{3 $\mathrm{Ba}^{+}$Natural Linewidth and the Doppler Shift}

Knowing this, the behaviour of the signal can now be examined under various experimental conditions. Several properties of the 3-level system are presented in table 5.2 , some of which will be used in performing calculations of $\rho_{a a}$. Since the signal is directly proportional to the excited state population, $\rho_{a a}$ it will suffice to examine 
Table 5.2: Vacuum wavelength $(\lambda)$, branching ratio, and spontaneous emission rate $(\Gamma)$ for energy level transitions in the Ba-II system.

\begin{tabular}{|c|c|c|c|}
\hline Transition & $\lambda(\mathrm{nm})$ & Branching Ratio $(\%)$ & $\Gamma\left(\times 10^{6} \mathrm{~s}^{-1}\right)$ \\
\hline \hline $6^{2} \mathrm{P}_{1 / 2} \rightarrow 6^{2} \mathrm{~S}_{1 / 2}$ & 493.54544 & $72.93 \pm 0.02$ & $92.1 \pm 0.9$ \\
$6^{2} \mathrm{P}_{1 / 2} \rightarrow 5^{2} \mathrm{D}_{3 / 2}$ & 649.86934 & $27.07 \pm 0.02$ & $34.2 \pm 0.3$ \\
\hline
\end{tabular}

${ }^{*}$ Values from $[19,53]$.

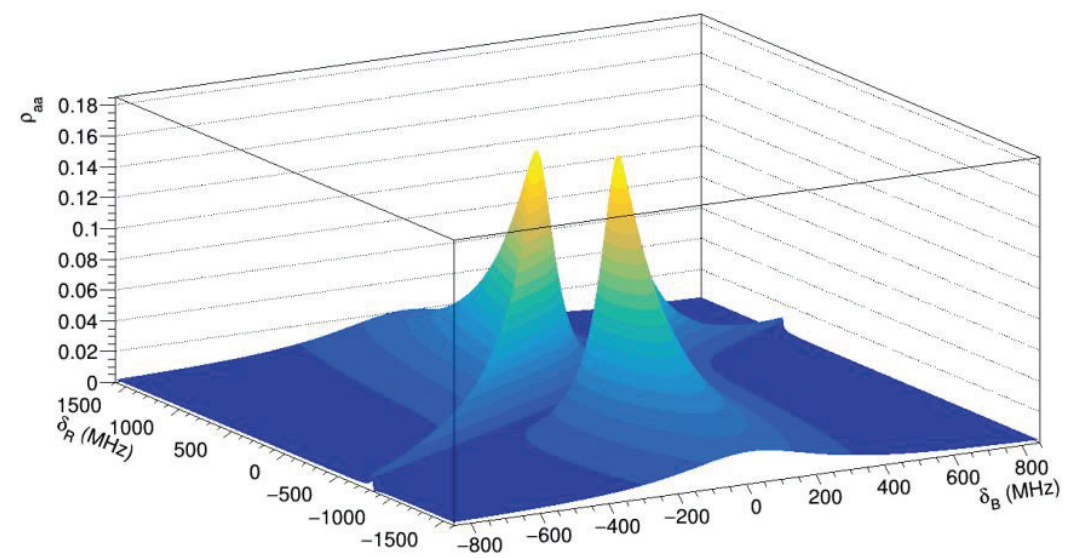

Figure 5.10: Excited state population, $\rho_{a a}$ as a function of $\delta_{\mathrm{B}}$ and $\delta_{\mathrm{R}}$. Here $\Omega_{\mathrm{B}}=$ $2 \pi(10 \mathrm{MHz})$ and $\Omega_{\mathrm{R}}=2 \pi(10 \mathrm{MHz})$.

the behaviour of this population. First the variation of $\rho_{a a}$ with $\delta_{\mathrm{B}}$ and $\delta_{\mathrm{R}}$ can be inspected. This is what is known as the natural linewidth and will be useful when optimizing the wavelengths of the lasers. A surface plot is shown in figure 5.10 showing $\rho_{a a}$ as a function of $\delta_{\mathrm{B}}$ and $\delta_{\mathrm{R}}$. Several cross sections are then shown in figure 5.11 showing $\rho_{a a}$ vs. $\delta_{\mathrm{B}}$ for four different values of $\delta_{\mathrm{R}}$. A projection along the other axis can be expected to look similar. Next the effects of varying the remaining parameters, $\Omega_{\mathrm{B}}$ and $\Omega_{\mathrm{R}}$, can be examined $\left(\Gamma_{\mathrm{B}}\right.$ and $\Gamma_{\mathrm{R}}$ are fixed properties of the system and will not vary). The excited state population, $\rho_{a a}$, vs. $\delta_{\mathrm{B}}$ is shown in figure 5.12 for several values of $\Omega_{\mathrm{B}}$.

It can be seen that up to a certain threshold, increasing $\Omega_{\mathrm{B}}$ leads to an increase in the peak excited state population however beyond this threshold the linewidth becomes broadened and the peak population decreases. At this point it should be 

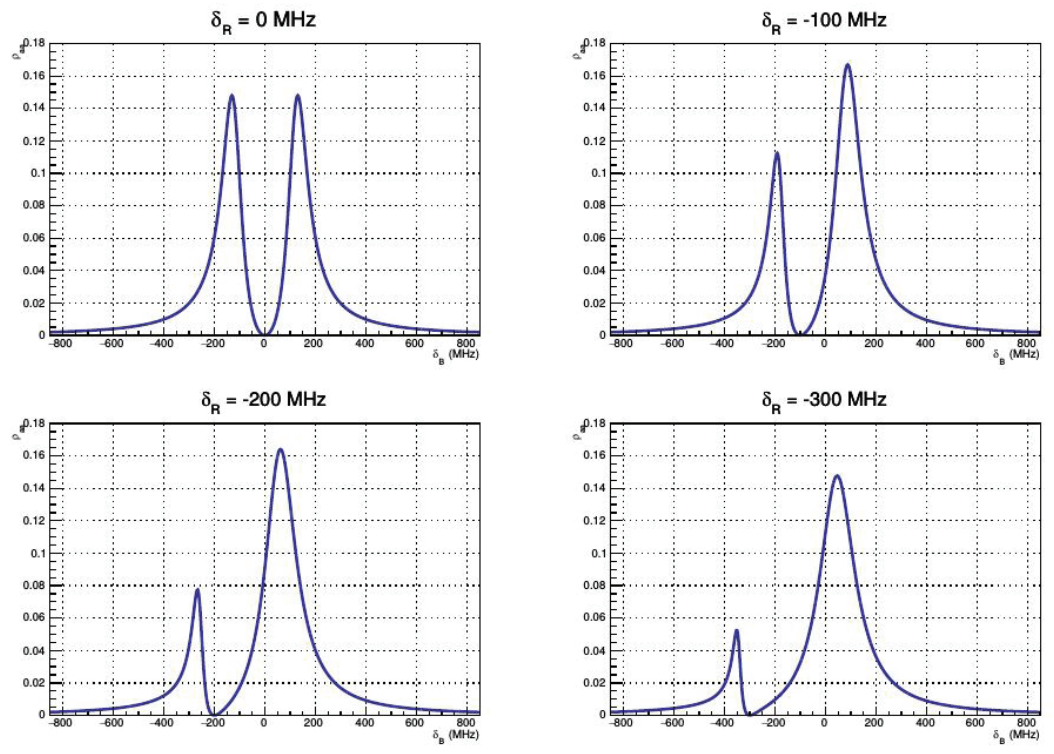

Figure 5.11: Excited state population, $\rho_{a a}$ as a function of $\delta_{\mathrm{B}}$ for different values of $\delta_{\mathrm{R}}$. Here $\Omega_{\mathrm{B}}=2 \pi(10 \mathrm{MHz})$ and $\Omega_{\mathrm{R}}=2 \pi(10 \mathrm{MHz})$.
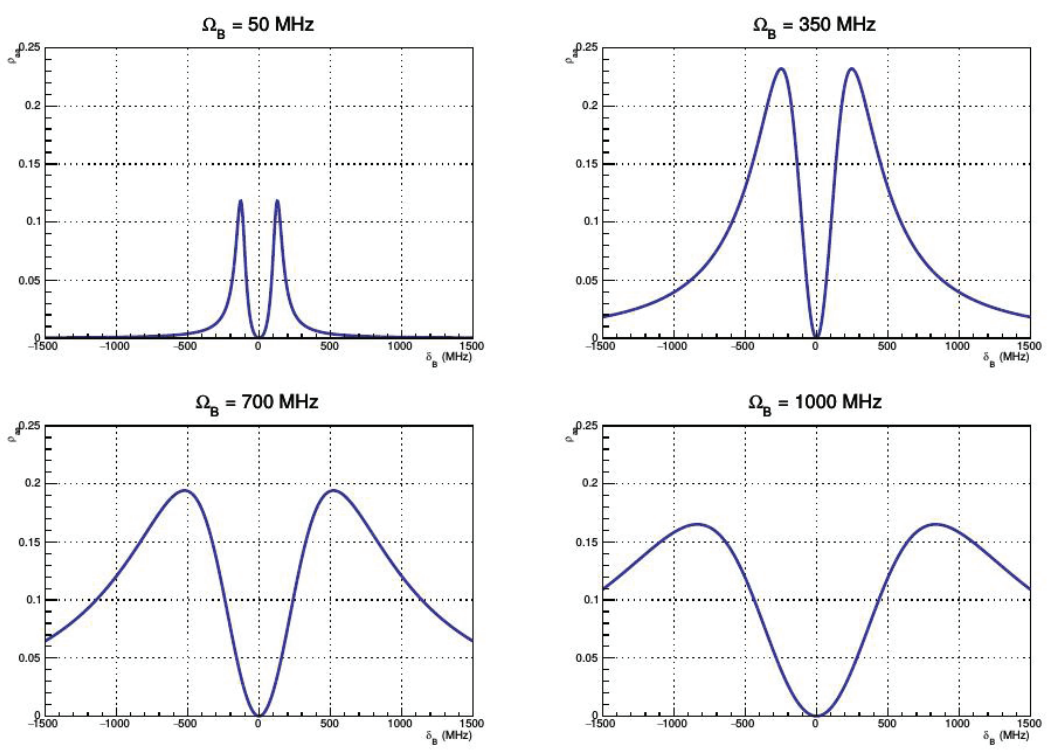

Figure 5.12: Excited state population, $\rho_{a a}$ as a function of $\delta_{\mathrm{B}}$ for different values of $\Omega_{\mathrm{B}}$. Here $\delta_{\mathrm{R}}=0 \mathrm{MHz}$ and $\Omega_{\mathrm{R}}=2 \pi(10 \mathrm{MHz})$. 
noted that,

$$
\left|\Omega_{\mathrm{B}, \mathrm{R}}\right|=\beta_{\mathrm{B}, \mathrm{R}} \frac{\sqrt{P_{\mathrm{B}, \mathrm{R}}}}{w_{\mathrm{B}, \mathrm{R}}}
$$

where $P_{\mathrm{B}, \mathrm{R}}$ are the beam powers, $w_{\mathrm{B}, \mathrm{R}}$ are the beam waists and $\beta_{\mathrm{B}}=184 \mathrm{~mm} \cdot \mathrm{MHz}$. $\mathrm{mW}^{-0.5}$ and $\beta_{\mathrm{R}}=169 \mathrm{~mm} \cdot \mathrm{MHz} \cdot \mathrm{mW}^{-0.5}$ are proportionality constants. It is seen that the Rabi frequencies, $\Omega_{\mathrm{B}, \mathrm{R}}$ depend on the laser powers and beam waists. With this information, the broadening effect is termed power broadening and will be used later to optimize the laser beam power for the experiment.

A final consideration which must be made is that realistically, the ions being observed are at non-zero temperature (i.e. they will be undergoing random thermal motion within the trap due to collisions with gas molecules). This motion will have the effect of Doppler shifting the incoming laser beam in the frame of reference of the ion. If the axis of the laser is defined as the z-axis then,

$$
\omega^{\prime}=\omega\left[1-\frac{v_{z}}{c}\right]
$$

where $\omega^{\prime}$ and $\omega$ are the shifted and unshifted frequencies of the laser, $v_{z}$ is the component of ion velocity along the z-axis and $\mathrm{c}$ is the speed of light. If for photon absorption it is required that $\omega_{0}=\omega^{\prime}$, where $\omega_{0}$ is the resonant frequency of the transition, then this can instead be written as a shift in the resonant frequency,

$$
\omega_{0}^{\prime}=\frac{\omega_{0}}{1-\frac{v_{\mathrm{z}}}{c}}
$$

which can then be rearranged to isolate $v_{\mathrm{z}}$,

$$
v_{\mathrm{z}}=c \frac{\omega_{0}^{\prime}-\omega_{0}}{\omega_{0}^{\prime}}
$$

Equation 5.50 can then be substituted into the Maxwell-Boltzmann velocity distribution to obtain a distribution for the Doppler shifted resonant frequency. If the distribution is rewritten in terms of a new detuning, $t=\omega_{0}^{\prime}-\omega_{0}$ it is found that,

$$
g(t)=\sqrt{\frac{1}{2 \pi \sigma^{2}}} \exp \left[-\frac{t^{2}}{2 \sigma^{2}}\right]
$$

where, 


$$
\sigma=\frac{\omega_{0}}{c} \sqrt{\frac{k_{\mathrm{B}} T}{m}}
$$

Here $T$ is the ion temperature, $m$ is the ion mass and $k_{\mathrm{B}}$ is the Boltzmann constant.

To see the effects this distribution will have on the linewidths, the distribution is convoluted with equation 5.44. To do this, consider the fact that the resonant frequency will be shifted for both transitions in the system,

$$
\begin{aligned}
& t_{\mathrm{B}}=\omega_{\mathrm{B}}^{\prime}-\omega_{\mathrm{B}}=\omega_{\mathrm{B}}\left(\frac{c}{v_{\mathrm{z}}}-1\right)^{-1} \\
& t_{\mathrm{R}}=\omega_{\mathrm{R}}^{\prime}-\omega_{\mathrm{R}}=\omega_{\mathrm{R}}\left(\frac{c}{v_{\mathrm{z}}}-1\right)^{-1}
\end{aligned}
$$

and note that $t_{\mathrm{B}}$ and $t_{\mathrm{R}}$ are actually linearly dependent and write,

$$
t_{\mathrm{R}}=\frac{\omega_{\mathrm{R}}}{\omega_{\mathrm{B}}} t_{\mathrm{B}}
$$

Now, $\rho_{a a}$ can be convoluted with the distribution, making note that in the equation for $\rho_{a a}$ the total detuning is a combination of the laser detuning from resonance with the detuning of the resonant frequency itself (i.e. $\delta \rightarrow \delta-t$ ).

$$
g_{\text {Doppler }}\left(\delta_{\mathrm{B}}, \delta_{\mathrm{R}}\right)=\sqrt{\frac{1}{2 \pi \sigma_{\mathrm{B}}^{2}}} \int_{-\infty}^{\infty} \rho_{a a}\left(\delta_{\mathrm{B}}-t_{\mathrm{B}}, \delta_{\mathrm{R}}-\frac{\omega_{\mathrm{R}}}{\omega_{\mathrm{B}}} t_{\mathrm{B}}\right) \exp \left[-\frac{t_{\mathrm{B}}^{2}}{2 \sigma_{\mathrm{B}}^{2}}\right] d t_{\mathrm{B}}
$$

This calculation can be performed numerically, the results of which are shown in figure 5.13 which depicts the Doppler broadened linewidth $\left(\rho_{a a}\right.$ vs. $\left.\delta_{\mathrm{B}}\right)$ for several different values of $\delta_{\mathrm{R}}$ at $298 \mathrm{~K}$. It is interesting to note that the central dip when $\delta_{\mathrm{B}}=\delta_{\mathrm{R}}$ no longer goes all the way to zero. This can now be used to optimize the experimental configuration. One method for this optimization is described in [19] however this is by no means the only way to optimize. Many numerical analyis software exist which have numerical integration and optimization methods which may also be used to determine the best combination of beam powers and frequencies to be used.

One further piece of information of interest here is the shift in resonant frequency 

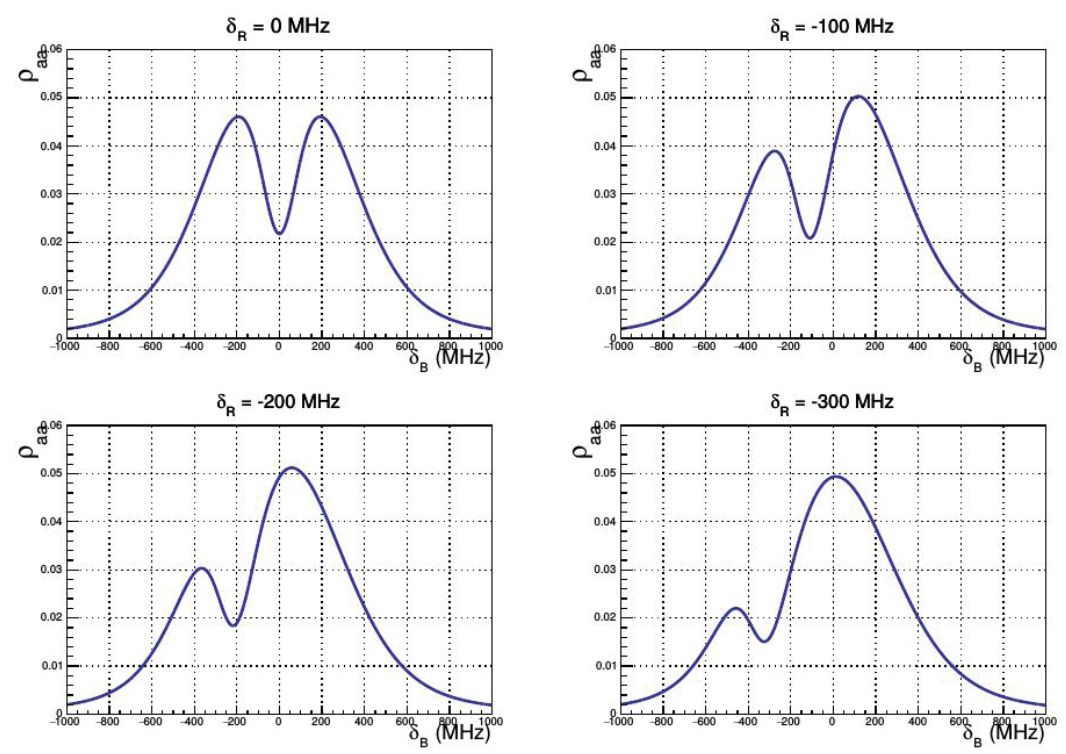

Figure 5.13: Excited state population, $\rho_{a a}$, with Doppler broadening at $298 \mathrm{~K}$ as a function of $\delta_{\mathrm{B}}$ for different values of $\delta_{\mathrm{R}}$. Here $\Omega_{\mathrm{B}}=2 \pi(10 \mathrm{MHz})$ and $\Omega_{\mathrm{R}}=2 \pi(10 \mathrm{MHz})$.

for different $\mathrm{Ba}^{+}$isotopes. The resonant frequencies used up to this point are those of ${ }^{138} \mathrm{Ba}$ however the isotope of interest in this experiment is ${ }^{136} \mathrm{Ba}$ which has measured isotope shifts of $\nu_{\mathrm{B}}^{138}-\nu_{\mathrm{B}}^{136}=179.4 \mathrm{MHz}$ [54] and $\nu_{\mathrm{R}}^{138}-\nu_{\mathrm{R}}^{136}=-68.0 \mathrm{MHz}$ [55]. While simply shifting the laser frequencies is not particularly exciting, this introduces the opportunity to not only count barium ions but also to identify which isotope is being looked at and also, given great enough sensitivity, to identify the composition of a sample of multiple ions at once. Figure 5.14 illustrates the shift in lineshape between ${ }^{136} \mathrm{Ba}$ and ${ }^{138} \mathrm{Ba}$ and also the combined lineshape in the case where two ions are present, one of each isotope. It must be noted that because of the hyperfine interaction, odd numbered isotopes are shifted significantly and will not be visible in the same frequency range as even numbered isotopes.

\subsubsection{Experimental Setup}

\section{Two-laser Spectroscopy}

In order to perform two-laser spectroscopy on a trapped barium ion, a pair of highly stable (in terms of both power and frequency) lasers is required, near the resonant frequency of each transition and with tuneable frequency so that the fluorescence 


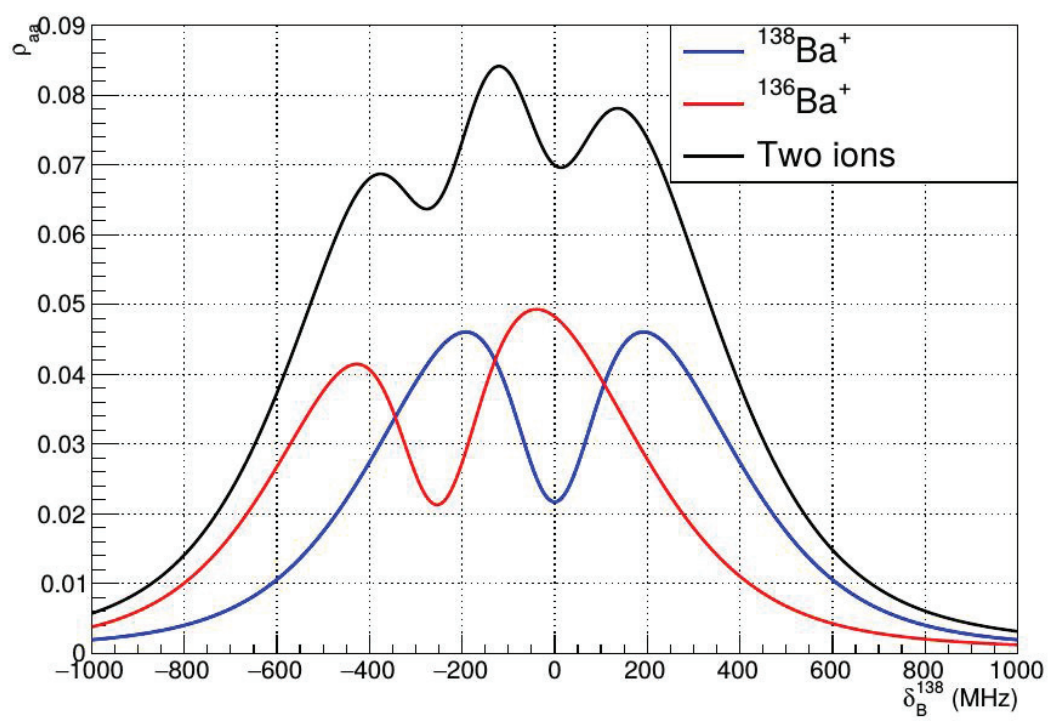

Figure 5.14: Doppler broadened linewidths for ${ }^{136} \mathrm{Ba}$ and ${ }^{138} \mathrm{Ba}$. Also shown is the combined linewidth when one of each isotope is present. Here $\Omega_{\mathrm{B}}=$ $2 \pi(10 \mathrm{MHz}), \Omega_{\mathrm{R}}=2 \pi(10 \mathrm{MHz})$ and $\delta_{\mathrm{R}}^{136}=0 \mathrm{MHz}$.

signal strength can be maximized.

The lasers chosen for this were both highly sophisticated diode lasers from TOPTICA; a TA pro [56] at $649 \mathrm{~nm}$ (red) and a TA-SHG pro [57] at $493 \mathrm{~nm}$ (blue). The configurations of both lasers are very similar (as illustrated in figure 5.15). A diode laser head [58] produces an infrared (IR) (or red, in the case of the red laser) beam at $\sim 986.7 \mathrm{~nm}$ (or $649 \mathrm{~nm}$ ) with a power of $\sim 1 \mathrm{~W}$ which is directed into a grating in the Littrow configuration. The first order diffracted beam from this grating is sent back into the diode in order to control the frequency of the laser. The zero-th order reflection from the grating now produces a weaker, frequency stabilized beam which is passed through an optical isolator, into a tapered amplifier and then through another optical isolator. The optical isolators protect the components from any possible back-reflection which could potentially cause damage or de-stabilize the laser frequency, and the tapered amplifier greatly increases the beam power. At this point the red laser passes through a shuttered output port, however the IR laser has some further optics in order to double it's frequency to achieve a blue laser beam at 493 $\mathrm{nm}$. This is necessary because there is no known diode that produces blue light at this wavelength.

The lasers in this state are already stable to within $10 \mathrm{MHz}$, however to more precisely match the resonance conditions of the Ba-II system some additional locking is 


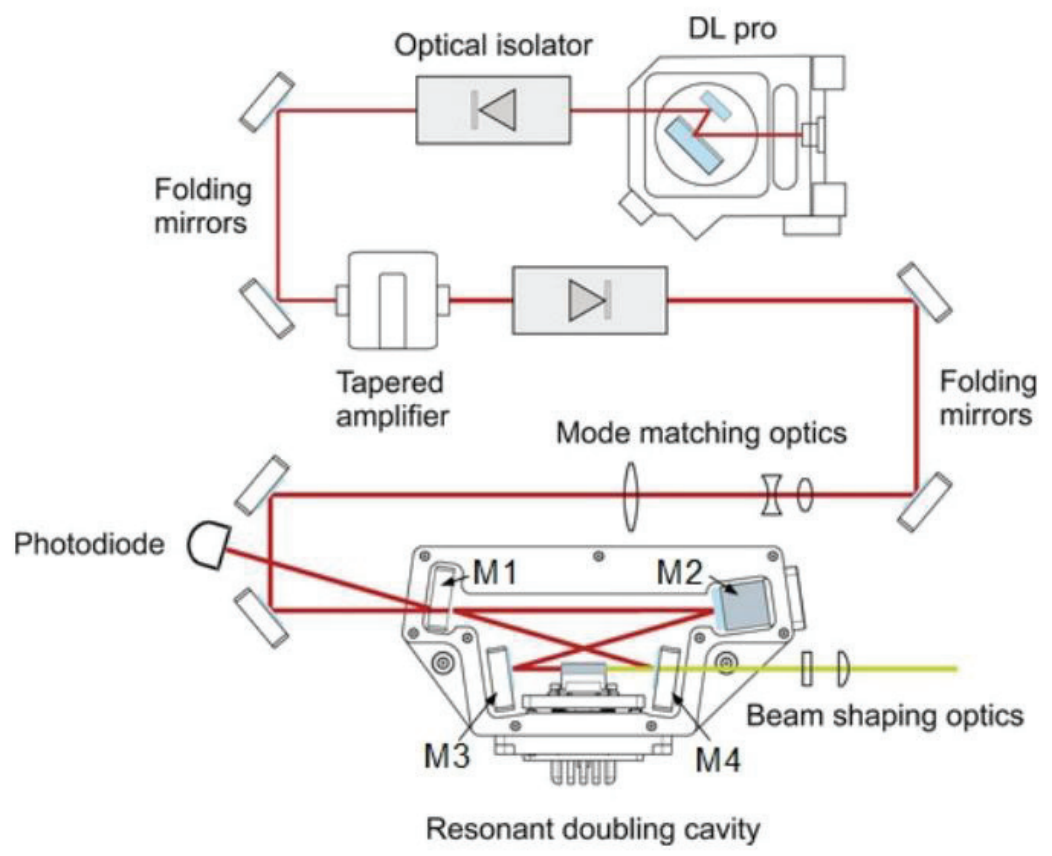

Figure 5.15: The components of a TOPTICA TA/SHG pro laser (the blue laser). In the case of a TA pro (the red laser), the beam output is after the second optical isolator and the rest of the components do not exist. Reprinted from [57].

used. Before the tapered amplifiers, each beam is sampled using a partially transmitting mirror. The sampled beams are fed through a fiber into a HighFinesse wavelength meter (WLM) [59] (using a fiber optic switch to alternate between the two beams). The wavelength meter utilizes a Fizeau interferometer to measure the wavelength of the input beams in reference to a frequency stabilized helium-neon (HeNe) laser [60] $(632.8 \mathrm{~nm})$. The wavelength meter provides locking settings through which a target wavelength is set and the WLM produces a PID (proportional-integral-derivative) locking signal which is fed back to the laser control electronics in order to lock the laser to the target frequency. With this locking technique, the lasers are now stable to within $1 \mathrm{MHz}$ and can be easily set to different frequencies near resonance (i.e. in order to measure linewidths).

In the TA/SHG pro, after the second optical isolator, the beam is directed through mode-matching optics into a resonant doubling cavity. The mode matching optics are required to match the beam to the fundamental mode of the cavity. The cavity itself (depicted in figure 5.15) consists of four mirrors (numbered M1-M4) and a secondharmonic-generator (SHG) crystal. The first mirror, M1 is a partially transmitting 
mirror, through which the beam enters the cavity. The beam is then steered by mirrors M2 and M3 into the SHG crystal. The output of the crystal is a mixed beam, mostly at the input frequency but with some small fraction of the power at twice the input frequency. The beam then reaches M4, another partially transmitting mirror, where the frequency doubled beam (now $493 \mathrm{~nm}$ blue light) is transmitted and the remaining IR beam is reflected back toward M1. A fraction of the beam power is transmitted through M1 back to a photo-diode which monitors the intra-cavity power, the rest of the beam is reflected back to M2 and follows the same path as the original beam through the cavity. A piezo crystal driven by a signal from a PoundDrever-Hall unit [61] controls the position of M2 in order to maintain resonance of the doubling cavity under fluctuations in temperature or air pressure using information from the photodiode. After the doubling cavity, the beam (now blue) passes through shaping optics to achieve a near diffraction limited Gaussian beam shape and is then passed through a shuttered output port.

When all components are properly aligned both lasers produce beam powers of around $\sim 200-250 \mathrm{~mW}$.

\section{Laser Modulation and Laser Beam Steering}

Figure 5.16 depicts the path of the laser beams leading from the laser outputs to the ion trap. While the blue laser contained shaping optics producing a Gaussian beam shape, the red did not and instead has a highly elliptical shape, therefore additional optics are required to shape the beam. The shaping optics consist of a pair of cylindrical lenses (with focal lengths of 50 and $75 \mathrm{~mm}$ ) on rotating mounts and an aperture. Using these lenses a proper Gaussian beam shape can be achieved for the red laser in order to match the blue, as shown in figure 5.17.

Once both beams are properly shaped, they are passed through a pair of acoustooptic modulators (AOMs). An AOM is an optical device consisting of a crystal subjected to a sound wave. The sound wave creates a wave pattern of varying refractive index inside the crystal which effectively acts as a diffraction grating. This effect can be exploited to treat the AOM as a high frequency beam shutter (the purpose of which will be explained later). With a properly designed and well aligned AOM, the majority of the beam power will be in the first order diffracted beam which will now be used as the primary beam, blocking the rest with a small aperture. If the 


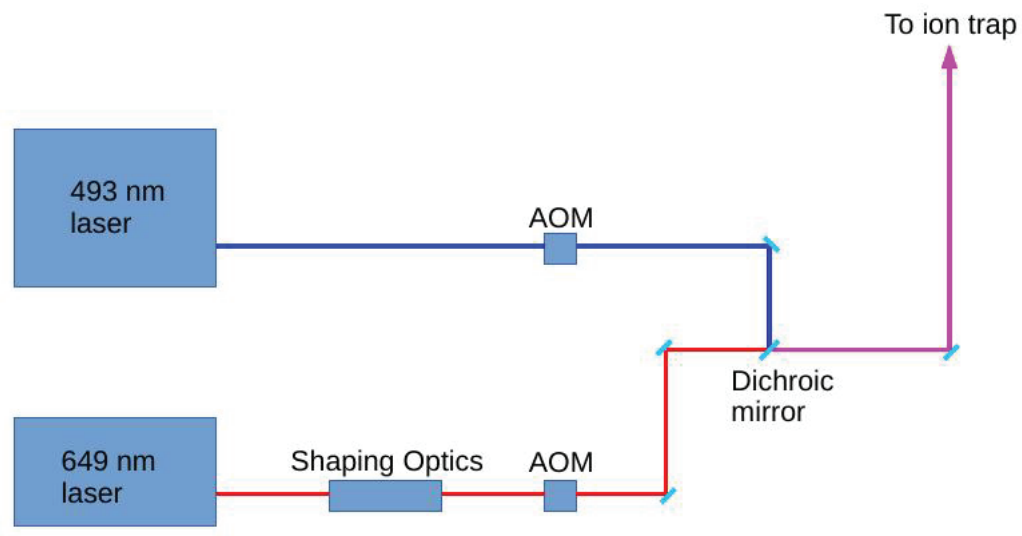

Figure 5.16: A schematic of the beam paths from the laser outputs to the ion trap.

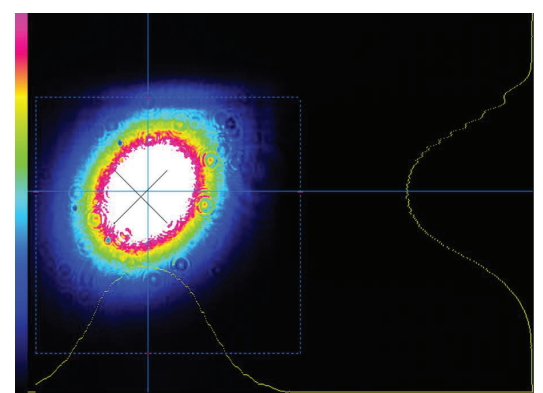

(a) Blue beam profile.

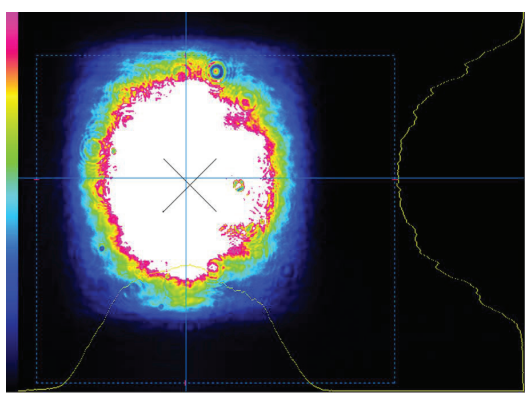

(b) Red beam profile.

Figure 5.17: Beam profiles of the blue and red lasers after shaping optics. Profiles were measured using a Newport LBP beam profiler [62]. 
sound wave in the crystal is then switched on and off (for example, by modulating it with a lower frequency square wave), then the first order diffracted beam will also be switched on and off. This technique was used to effectively shutter the laser beams at frequencies up to $\sim 200 \mathrm{kHz}$. It must be noted that AOMs cause a shift in the frequency of the laser beams equal to the frequency of the sound waves $(87.5 \mathrm{MHz}$ for the blue laser, $80.0 \mathrm{MHz}$ for the red) which must be accounted for when setting the laser frequencies.

A limitation of this shuttering technique is that AOMs have an intrinsic risetime, in other words, the amount of time it takes for the beam to fully switch on/off. This risetime is proportional to the beam diameter within the crystal $(110 \mathrm{~ns} / \mathrm{mm}$ for the blue AOM [63] and $150 \mathrm{~ns} / \mathrm{mm}$ for the red [64]) and so it is helpful to focus the beams into the AOMs (with the beam waist inside the crystal) thereby greatly reducing the risetime. To do this, a pair of short focal length lenses is used to focus the laser beams (locating the focal point in the centre of the AOM crystals), achieving a beam waist of $\sim 0.15 \mathrm{~mm}$ for the blue beam and $\sim 0.06 \mathrm{~mm}$ for the red, corresponding to risetimes of $\sim 20$ ns and $\sim 10$ ns respectively. After the AOMs, a second pair of lenses is used to re-collimate the beams.

After the AOMs, the newly re-collimated beams are each passed through a polarizer and a quarter-wave plate on a rotating mount. Thanks to the polarized nature of laser light, the polarizers can be used to control the power of the laser beams. The power is set by measuring the beam power with a Newport power meter [65] and rotating the polarizer until the desired power is reached. The quarter-wave plate then changes the linearly polarized laser light to circularly polarized to avoid any polarization dependence later in the system.

After the beam powers are set, the beams are steered with a set of mirrors to a dichroic mirror (reflective to blue, transmissive to red) where they are overlapped into a single beam. Proper alignment of the beams is achieved using a pair of mirrors to steer each beam through the same set of apertures, maximizing the transmitted beam power through each aperture (measured using the power meter).

Once overlapped, a set of mirrors is used to steer the beam into the ion trap, with the beam axis aligned to the z-axis of the trap, entering through a window located by the trap exit. The beam is carefully aligned so as to pass directly through the centre of the trap in order to minimize the amount of light scattered from the electrodes and to have the greatest beam intensity at the centre of the trapping region where 
Table 5.3: Specifications of the Hamamatsu PMT used for light collection.

\begin{tabular}{|c|c|}
\hline Parameter & Value \\
\hline \hline Photocathode material & GaAsP \\
Photocathode dimensions & $5 \mathrm{~mm}$ diameter \\
Quantum efficiency & $\sim 0.37493 \mathrm{~nm}$ \\
Dark counts & $300 \mathrm{~s}^{-1}$ \\
Max. current-output & $2 \mu \mathrm{A}$ \\
\hline
\end{tabular}

*Values from [66].

the trapped ions are expected to be.

\section{Light Detection}

To detect the fluorescence signal from the $\mathrm{Ba}^{+}$ions, a current-output type Hamamatsu photomultiplier tube (PMT) [66] is used, the specifications of which can be found table 5.3. The PMT was placed directly above the trapping segments (segments 13 and 14) of the ion trap, facing it through a window, in order to collect as much of the fluorescence signal as possible.

To maximize the light collected, a mirror was mounted directly below the trapping segments, opposite the PMT, to reflect light back up. A lens was also attached to the PMT to improve light collection, furthermore, a $24 \mathrm{~nm}$ band-pass filter, centered at $493 \mathrm{~nm}$, was attached in order to block out any possible background light. The overall light collection efficiency of this setup was estimated in [19] to be $\sim 0.7 \%$.

The output of the PMT comes in the form of current pulses which can be measured using two possible techniques. The first technique is to connect the PMT output to a Keithley microammeter [67] which is used to convert the current to a voltage signal which is then displayed on a digital oscilloscope [68]. This technique is useful for alignment and simple optimization work because it provides easily interpreted, instant feedback, however it is not very sensitive and so is not useful for precision measurements such as ion counting.

In the second technique, the PMT signal is sent to an amplifier/discriminator [69] which converts the current signal to a series of TTL pulses. These TTL pulses are then sent to a multichannel scaler (MCS) [70] to be counted into a series of bins, 
each corresponding to a different time. The details of analyzing the MCS data will be explained later.

\subsection{Measurement Technique and Old Results}

\subsubsection{Laser Ablation Ion Source}

In order to test the barium ion detection system, a reliable source of barium ions is required. To this end, a simple laser ablation ion source was designed using minimal lab equipment.

The source itself is a small chunk of barium metal placed inside the ion trap vacuum chamber near the entrance of the trap. To produce barium ions, a pulsed UV laser [71] is shone through a viewport and focused onto the surface of the barium chunk. The laser pulses eject ions as well as neutral barium from the surface of the metal, some of which find their way into the ion trap.

In practice, laser ablation creates far more ions than needed for the experiment and a method was discovered which allows production of a much smaller number of ions with no additional equipment. This method was originally discovered as the result of diagnosing a problem with the experiment. It was observed that even when not ablating the barium, so long as the ion trap was turned on, there was a slow increase in signal over time. Eventually it was found that the pressure gauges [45] used to monitor the vacuum pressure were the cause of this. As a side effect of their regular operation, the gauges ionize some of the barium present in the chamber.

The pressure gauges are now used to create ions from the neutral barium present in the vacuum chamber. During normal operation the gauges are disconnected to avoid this "self-loading" effect that had been causing problems, however when ions are needed one of the gauges is connected until enough trapped ions are observed. Using this technique, it is possible to introduce very small numbers of ions into the trap in a somewhat controlled manner (generally $<5$ ions $/$ sec from pressure gauge). A small disadvantage to this is that it disallows monitoring of the system pressure while making measurements, however it has been found that the pressure is reasonably stable over time, so constant monitoring is not necessary. 


\subsubsection{Laser Alignment}

In order to properly align the lasers into the ion trap, the trap is first loaded with many ions using the laser ablation source (no precise amount required, 2-3 $25 \mu \mathrm{J}$ pulses usually produce a sufficient number) and the signal is monitored using an oscilloscope [68] as previously explained.

The red laser is then modulated (typically at $100 \mathrm{~Hz}$, although the frequency is not critical for this) creating a square wave pattern on the oscilloscope in which the peaks, when the red laser is on, correspond to signal + background, and the troughs, when the red laser is off, correspond to background only. While monitoring this pattern, the alignment is adjusted (using a series of mirrors on 2-axis tilt mounts) to maximize the signal-to-background ratio.

\subsubsection{Measurement Techniques}

In order to count ions within the trap, two different measurement techniques have been developed, both of which utilize the TTL/counting photon detection method previously mentioned. The first technique is called continuous excitation while the second is called intermodulation. The name of each technique corresponds to the pattern in which the lasers are modulated. Both techniques return a photon count rate as signal. In order to actually count the number of ions, the expected count rate for a single ion is determined and then the total signal should be approximately an integer multiple of this rate.

\section{Continuous Excitation}

The continuous excitation technique is very similar to the measurement used for laser alignment. The blue laser is left on continuously, pumping the $6^{2} \mathrm{~S}_{1 / 2} \rightarrow 6^{2} \mathrm{P}_{1 / 2}$ transition while the red laser is cycled on and off at $100 \mathrm{~Hz}$ with a $50 \%$ duty cycle. The laser duty cycle is illustrated in figure 5.18.

As explained in the alignment procedure, while the red laser is off, any light collected (mainly scattered light from the blue laser) is considered background, whereas light collected with the red laser on is considered signal + background. Now it is a simple process to subtract the background to find the total signal and if the total integration time for one multichannel scaler (MCS) scan is known then it is easy to 


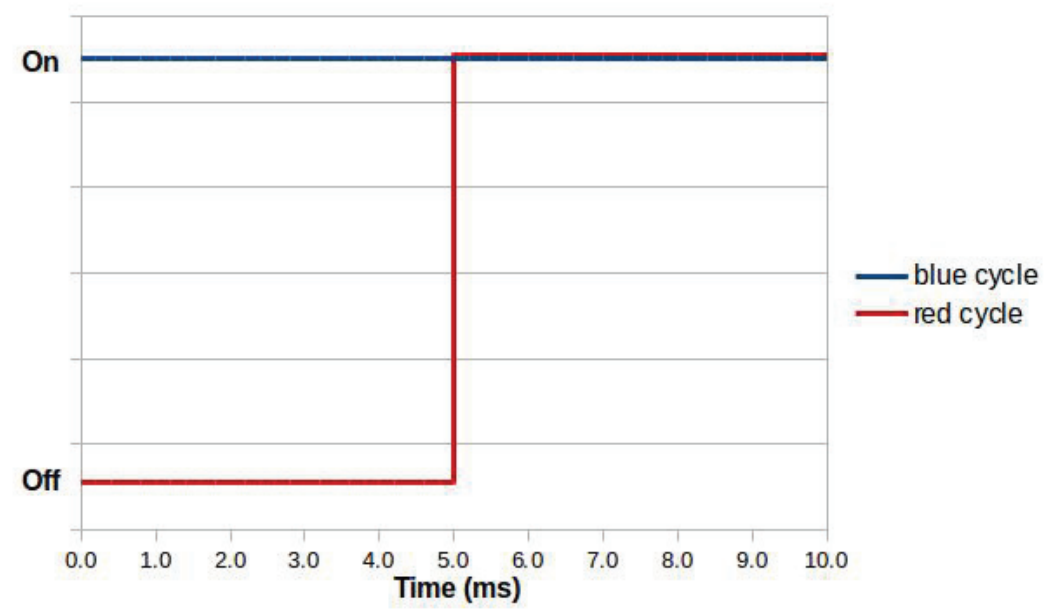

Figure 5.18: Red and blue laser duty cycles during continuous excitation counting technique. The blue laser is held on continuously and the red laser is pulsed on and off with a $50 \%$ duty cycle.

convert a number of photons into a fluorescence rate.

The MCS settings can be examined in further detail. In both measurement techniques, the MCS is configured so that there are 50 bins and so that a complete scan takes 1 second. To choose the bin width, $t_{\text {bin }}$ (i.e. how much time each bin covers) the length of a full laser cycle is divided by the number of bins, in this case $0.01 \mathrm{sec} / 50 \mathrm{bins}=0.2 \mathrm{~ms}$. Finally the number of cycles to sum over for a full scan, $n_{\text {cycles }}$, can be set. This is just $1 /$ time per cycle (i.e. \# of cycles = cycle frequency $/ 1 \mathrm{~Hz}$ ), in this case 100 cycles. An image of a full scan using this technique is shown in figure 5.19.

When analyzing a scan, $\sim 25$ bins will correspond to signal + background, $S+B$, and the remaining $\sim 25$ correspond to background, $B$, only. Some bins may have only partial signal strength due to the risetime of the AOMs. The fluorescence photon rate is now $[(S+B)-B] /\left(25 t_{\text {bin }} n_{\text {cycles }}\right)=[(S+B)-B] / 0.5 \mathrm{~s}$.

\section{Intermodulation}

The second technique uses a slightly more complicated modulation scheme. In this method both lasers are modulated at $200 \mathrm{kHz}$, the blue laser with a $60 \%$ duty cycle and the red laser with a $6 \%$ duty cycle. The duty cycles are timed such that there is $1.7 \mu$ s deadtime after the blue laser turns off and before the red laser turns on. 


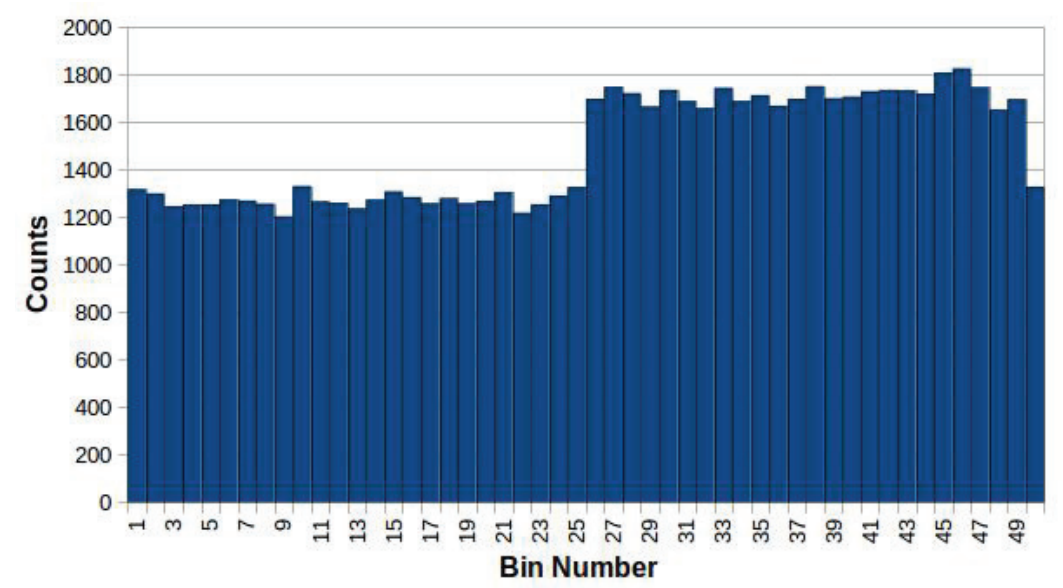

Figure 5.19: A single MCS scan using the continuous excitation measurement technique. Each bin constitutes $0.02 \mathrm{~s}$. In the first half of the scan, the red laser is turned off and there is only background scattered light. In the second half, the red laser is turned on and signal is observed.

The time dependence of this system has been studied in detail in [19], suffice to say that while the blue laser is on, all ions are pumped into the meta-stable state where they remain until the red laser is turned on at which point they are pumped into the excited state and quickly decay back to ground. The laser cycles are depicted in figure 5.20. The main advantage of this technique over the continuous excitation is that now the blue laser is not on during the period of signal collection, almost entirely eliminating background counts when no barium is trapped (confirmed via testing).

Similar to what was done for the continuous excitation method, the bin width can be calculated, $t_{\text {bin }}=0.1 \mu \mathrm{s}$, and number of cycles, $n_{\text {cycles }}=2 \times 10^{5}$. The scan, an example of which is shown in figure 5.21, is now more complicated than it was for the continuous excitation method. The first 30 bins (60\% duty cycle) correspond mostly to scattered light from the blue laser, there are then 17 bins during which neither laser is turned on. The counts in these bins appear to follow a sort of exponential decay trend and it has been determined that this is the PMT cooling after the period of intense blue light. Finally, the last three bins, corresponding to the red laser being turned on, give the signal + background counts. The three bins immediately before the red laser has turned on are treated as background since by that point the PMT has mostly finished cooling. Now the signal count rate is $[(S+B)-B] / 3 t_{\text {bin }} n_{\text {cycles }}=$ $[(S+B)-B] / 0.06 \mathrm{~s}$. 


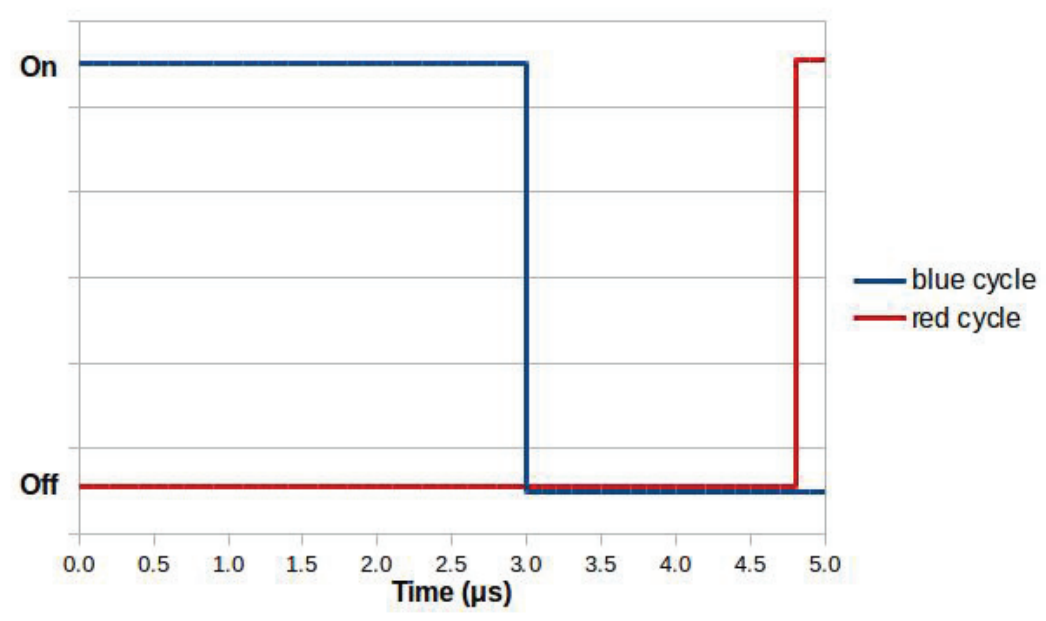

Figure 5.20: Red and blue laser duty cycles during the intermodulation counting technique. The blue laser is held on for the first $60 \%$ of the cycle, it is then switched off and the red laser is turned on for the last $6 \%$.

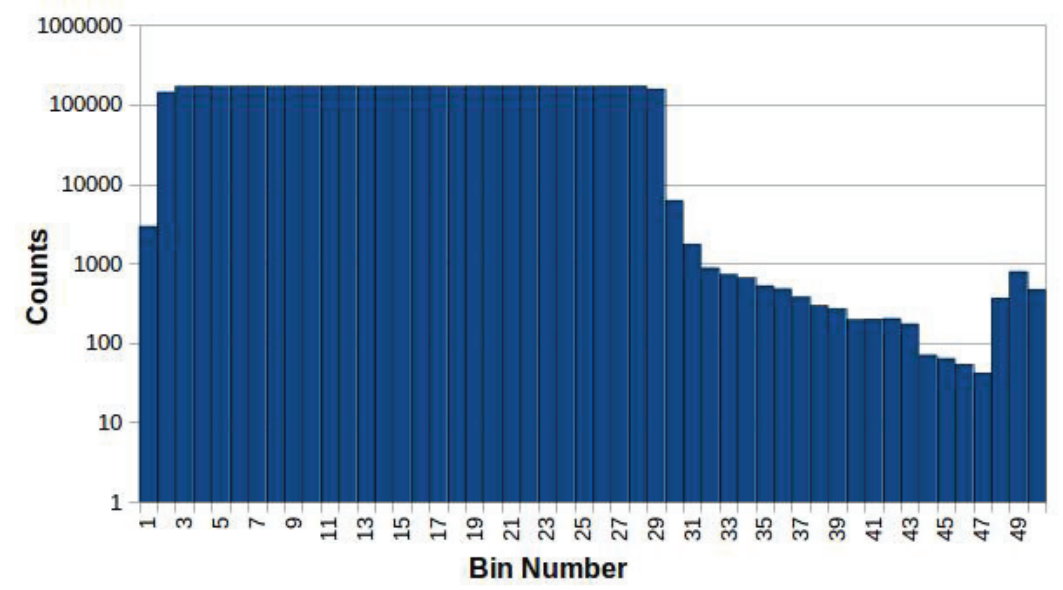

Figure 5.21: A single MCS scan using the intermodulation measurement technique. Each bin spans $0.02 \mathrm{~s}$. The first 30 bins show scattered light while the blue laser is on. The following 17 bins show a gradual decrease is signal as the PMT cools, and the final 3 bins show signal while the red laser is on. 


\subsubsection{Ion Counting Results}

Results of ion counting using the aforementioned techniques have been presented in [19]. It was shown that the intermodulation technique produces clearly visible discrete steps between different numbers of ions with single ion signal rate of $\sim 50 \mathrm{~Hz}$ and a background rate of $\sim 70 \mathrm{~Hz}$. Two examples of these discrete steps are shown in figure 5.22. The first observation (figure 5.22(a)) shows four ions exiting the trap one-by-one with a signal-to-noise ratio (SNR) of 4.6 and a signal to background (S/B) ratio of 0.16 on the steps in photon rate. The second observation $(5.22(\mathrm{~b}))$ similarly shows three ions with a SNR of 8.8 and S/B of 0.76. Unfortunately there was no such success using the continuous excitation method. 


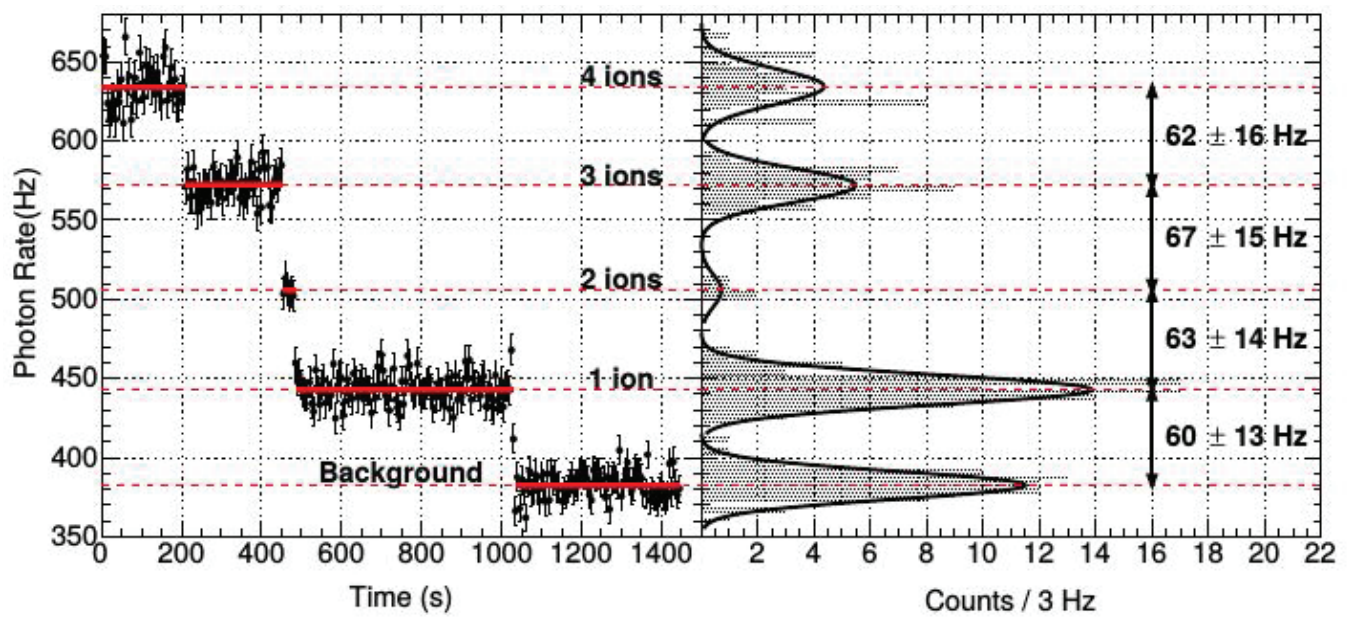

(a)

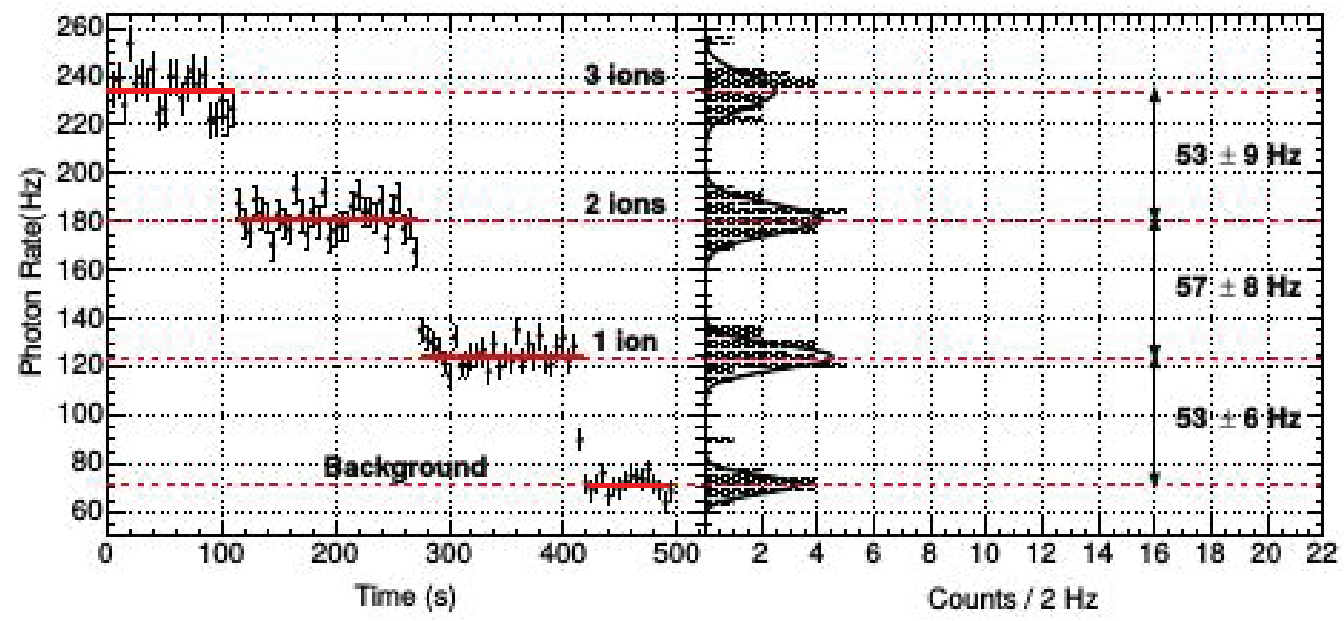

(b)

Figure 5.22: Previous barium tagging results. Discrete steps in photon count rate indicate individual ions exiting the trap. Figure 5.22(a) shows a SNR of $\sim 4.6$ and $\mathrm{S} / \mathrm{B}$ of $\sim 0.16$. Figure $5.22(\mathrm{~b})$ shows a $\mathrm{SNR}$ of $\sim 8.8$ and $\mathrm{S} / \mathrm{B}$ of $\sim 0.76$. Both figures reprinted from [19].

\subsection{Improvements to system}

One of the major limiting factors of the continuous excitation technique is the fact that the blue laser is turned on continuously. Because of this, the majority of the observed blue light is just scattered laser light rather than fluorescence. Since the number of photons observed follows a Poisson distribution, the noise seen on a scan 


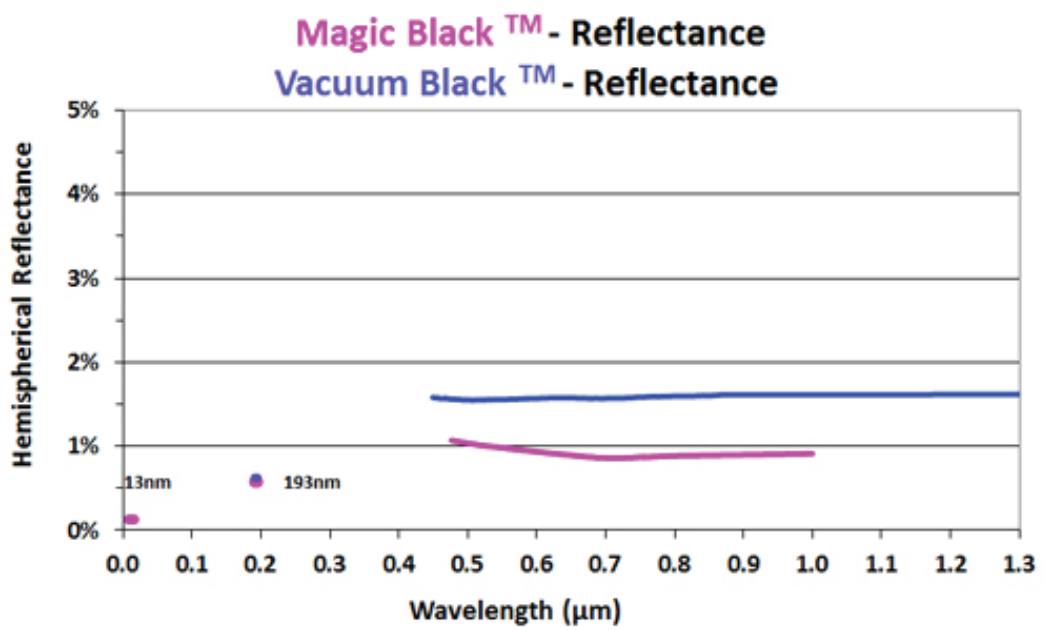

Figure 5.23: The hemispherical reflectance of Acktar Magic Black ${ }^{\mathrm{TM}}$ and Vacuum Black $^{\mathrm{TM}}$, another ultra-black coating which was considered. Reprinted from $[72]$.

is expected to be the square-root of the number of photons (where the total number is simply signal + background counts), thus with such a large background the signal becomes lost in noise. In the interest of seeing real results with this technique, some steps have been taken to minimize the amount of light that is scattered off the trap electrodes.

\subsubsection{Anti-reflective Black Coating}

One such step toward this background reduction was to have some of the electrodes covered in an ultra-black anti-reflective coating. The coating chosen was Acktar Magic Black $^{\mathrm{TM}}$, a carbon microstructure vacuum coating with extremely low hemispherical reflectance across a wide range of wavelengths (see figure 5.23). Segments 12-15 of the ion trap were coated (i.e. the trapping region and neighboring segments) while the rest were not.

A number of physical properties of the coating are listed in table 5.4. The only major drawback with this particular coating is the light abrasion resistance. Figure 5.24 shows a photograph of the coated electrodes in which some scratches on the coating are visible. This damage was sustained from only light handling while installing the electrodes in the trap. 
Table 5.4: Some physical properties of Acktar Magic Black ${ }^{\mathrm{TM}}$.

\begin{tabular}{|c|c|}
\hline Property & Value \\
\hline \hline Coating thickness & $4-7 \mu \mathrm{m}$ \\
Working temperature & $-269^{\circ} \mathrm{C}$ to $+350^{\circ} \mathrm{C}(4 \mathrm{~K}$ to $623 \mathrm{~K})$ \\
Weight of coating & $1.1-1.6 \mathrm{mg} / \mathrm{cm}^{2}$ \\
Abrasion resistance & light \\
Outgassing & CVCM $0.000 \%$, RML $0.2 \%$ \\
Chemical content & completely inorganic \\
Cleanability & Withstand cleaning with ethanol, IPA or acetone \\
\hline
\end{tabular}

${ }^{*}$ Values from [72].

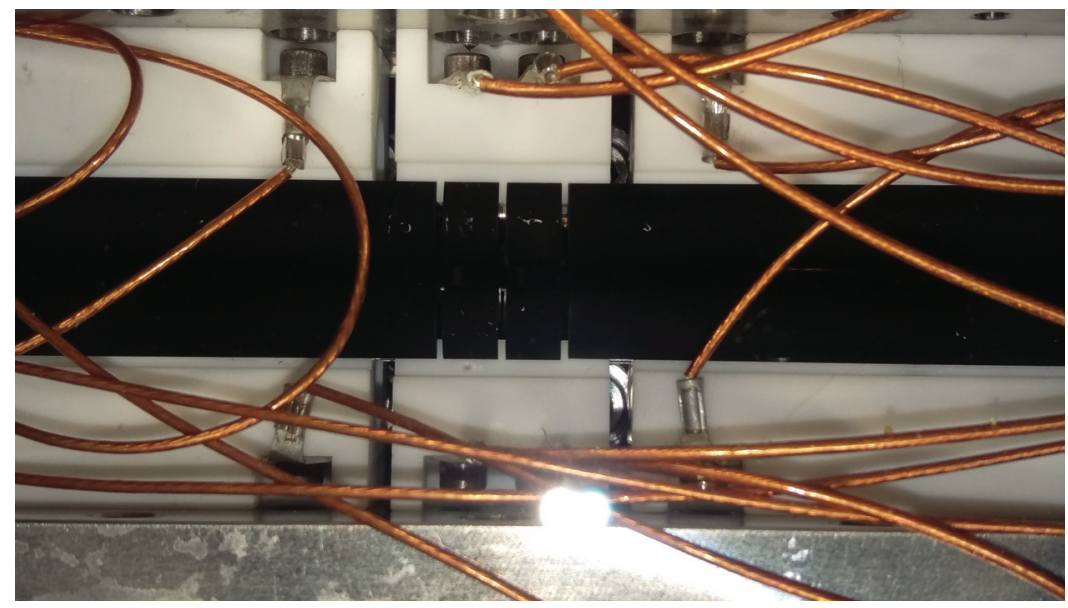

Figure 5.24: Photograph of trap electrodes coated with Acktar Magic Black ${ }^{\mathrm{TM}}$. Some rods show minor damage to the coating due to handling during installation. 


\subsubsection{Optics Improvements}

One other change made to reduce scattered light was the introduction of a focusing lens near the trap window. This lens focuses the laser beam, thereby reducing the beam waist which in turn reduces the amount of light scattering off the trap electrodes. Furthermore, by reducing the beam waist while maintaining the same total power, the beam intensity is actually increased, potentially increasing the signal (limited by saturation). A long focal length lens ( $500 \mathrm{~mm})$ was used because a shorter length will actually lead to additional scattered light due to the rapid widening of the beam beyond the focal point.

\subsubsection{Improved Results}

With these improvements to the background reduction, significantly better results were seen using the intermodulation technique. The new results are shown in figure 5.25 and when compared to those shown previously in 5.22 there are a few noticeable differences. First, the photon counting rate has gone up significantly for both signal and background. This difference appears to be a simple scalar multiplication and has been attributed to either a difference in how the number of photons was converted to a counting rate, or improvements in the alignment of the counting optics, allowing more efficient light collection. The second major difference is the increase in S/B ratio. This was calculated by fitting a gaussian function to each peak the histogram of photon count rates and taking $\mathrm{S}$ to be the average step size between different numbers of ions and B to be the mean value of the lowest plateau. Previously the results (only considering the best result in figure 5.22(b)) showed a signal of $53 \mathrm{~Hz}$ and a background of $\sim 70 \mathrm{~Hz}$, giving $\mathrm{S} / \mathrm{B}=\sim 0.76$. The new result now has a signal size of $\sim 2300 \mathrm{~Hz}$ and background of $\sim 2000$ giving a S/B ratio of $\sim 1.15$, a relative improvement of $\sim 50 \%$ over the previous result. The signal-to-noise ratio (SNR) was also calculated by dividing each step size by it's corresponding error (from the standard deviation of the fit results). Converse the S/B, the SNR of the new result was found to be $\sim 5.6$, falling roughly in between the two previous results.

In addition to the improvements seen using the intermodulation technique, it is now possible to see single ion sensitivity with the continuous excitation method. While the results are still much worse than those from intermodulation, a semblance of discrete steps can be seen in figure 5.26 using this technique. The ion steps shown 


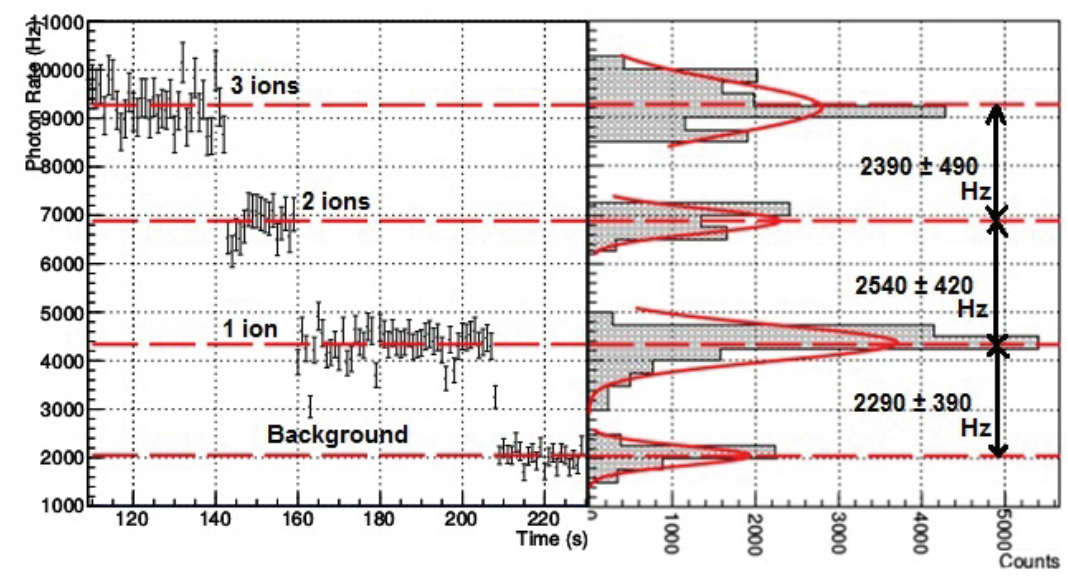

Figure 5.25: Discrete ion steps found using the intermodulation technique after improvements to background reduction in the ion trap. The photon rate of each ion step and their corresponding noise are found by fitting the photon count rates of each step to a gaussian function (shown on the right). This scan shows a SNR of 5.6 and S/B of 1.2.

here show a magnitude of the same order as those shown by the intermodulation technique however due to the higher background level, the steps are too swamped by noise to easily count. Upon examination of the histogram of photon rates, one can pick out what appear to be peaks corresponding to different numbers of $\mathrm{Ba}^{+}$ions. Unfortunately these peaks are poorly resolved from one-another to the extent that they could not be fit with gaussian functions. By visual inspection it can be gathered that the separation of these peaks is $<1 \sigma$, much too little for efficient ion counting. The current ion trap is approaching a limit at which further background reduction will likely be impossible and thus a new ion trap is being devised. The plans for the new trap were originally begun at Carleton University and now the main design stage is taking place at TRIUMF. This new trap will utilize a bladed quadrupole design (see figure 5.27) in which, rather than cylindrical rods, a blade shaped electrode is used. This design will allow for greater access to the trapping region from the sides of the trap, allowing the lasers used for optical tagging to be directed in from the side, rather than longitudinally. This will decrease the number of surfaces encountered by the lasers and thus greatly decrease the amount of scattered laser light (hence, greatly decreasing scattered light background). Figure 5.27 shows the new ion trap design as well as the design of the ion trap at Innsbruck upon which it was based [73]. 


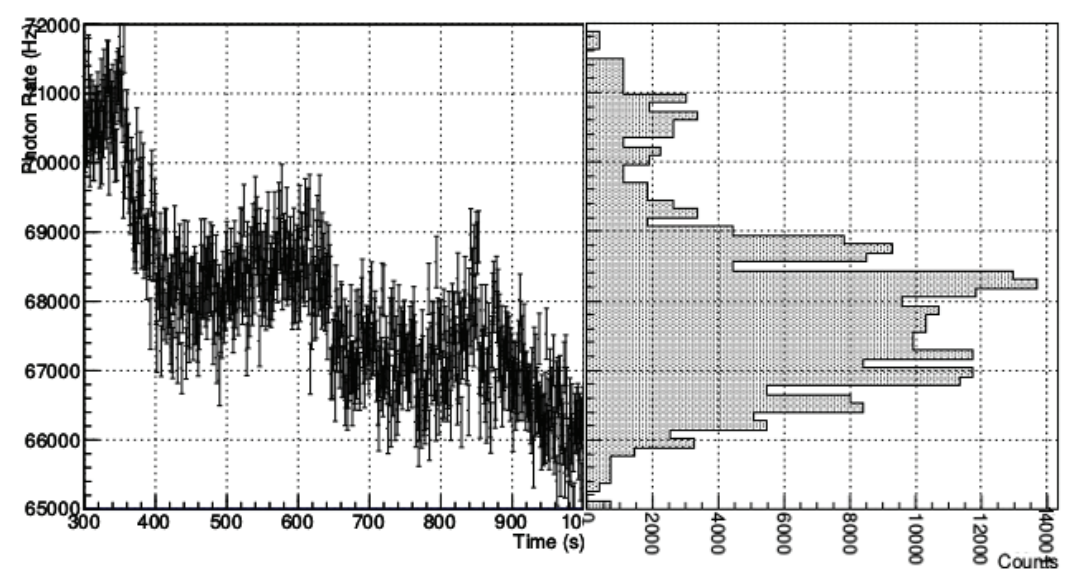

Figure 5.26: New ion counting attempt using the continuous excitation method. There are hints of discrete ion steps, however still not as good as the intermodulation method.

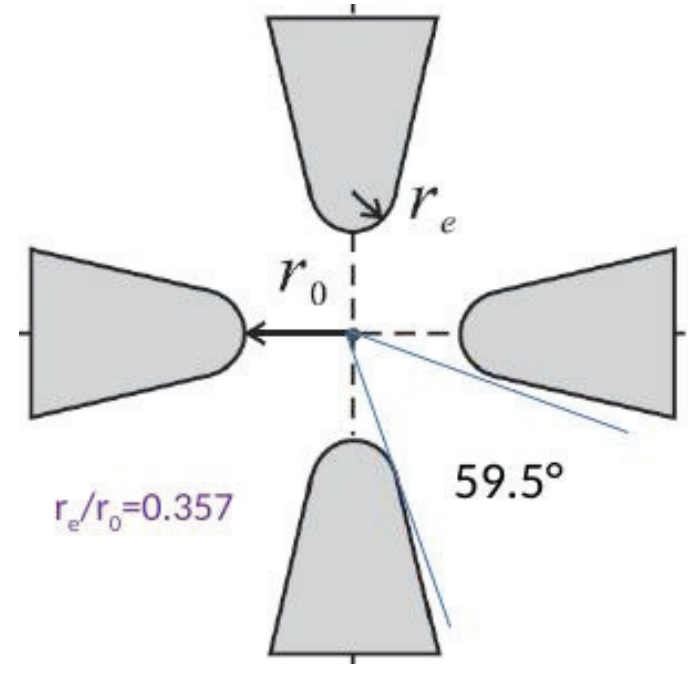

(a)

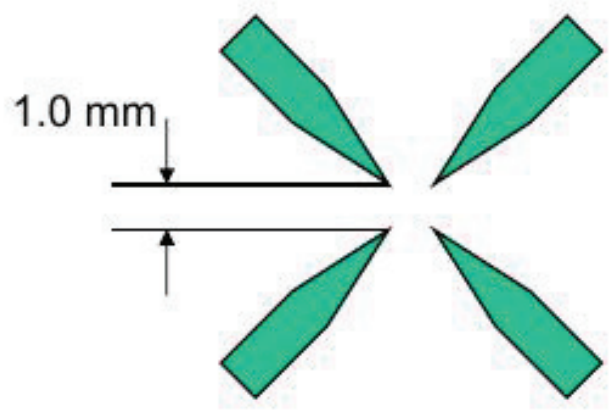

(b)

Figure 5.27: Figure 5.27(a) shows the design of a new bladed quadrupole ion trap and figure 5.27(b) shows the design of a similar trap used at Innsbruck upon which the new design was based. This new bladed design is in the design state at TRIUMF and is intended to replace Carleton's ion trap for use in barium tagging when completed. Figure 5.27(a) reprinted from [74] and figure 5.27(b) reprinted from [73]. 
With the large active volume of the $\mathrm{nEXO}$ detector and $\beta \beta$ event (either $2 \nu$ or $0 \nu$ ) is expected to occur roughly every 4.5 seconds, therefore it is important that the barium tagging apparatus is able to quickly capture and identify the barium daughter ions. While the time taken for the ions to become trapped was not studied in this work, some investigation has been done in [13]. The studies shown in this work as well [19] and [13] each show the ability to count ions via optical tagging. The SNR found in each study was sufficient that a single ion count rate and the empty trap background are separated by $>4 \sigma$ using measurements as short as one second. At this significance level (> 99.994\% certainty), only one or two measurements are necessary for single-ion sensitivity without false $\mathrm{Ba}$ ion observation and without missing the ion. Furthermore, at a rate of $\sim 1$ s per scan, this can be achieved at a high enough speed to easily keep up with the event rate of nEXO.

\subsection{Isotope Tagging}

One final study which may be performed using the ion trap and optical tagging apparatus is to experimentally observe the $\mathrm{Ba}^{+}$linewidths shown in figure 5.13. This is useful in barium tagging because the location of the central destructive interference in the $\mathrm{Ba}^{+}$linewidth is dependent on the Ba-isotope mass. If this interference were to be observed, it could be used to distinguish ${ }^{136} \mathrm{Ba}$ from other isotopes which could potentially trigger a false positive reading (mainly ${ }^{138} \mathrm{Ba}$, the most abundant isotope of barium consituting $\sim 72 \%$ of all natural barium). This can conceptually be done with the apparatus simply by taking a series of signal measurements while varying the laser frequency (by modifying the locking setpoint). Some attempts have been made toward this goal and have been met with mixed success.

With the intermodulation method, only one laser field is present at a time so the optical Bloch equations are changed and the double peak pattern is not observed, hence continuous excitation must be used. Unfortunately, the continuous excitation technique is not yet consistently sensitive to single ions and therefore, linewidth measurements must be taken with multiple ions present in the trap (usually a large number similar to the amount used during laser alignment). Although linewidths can be observed in this manner, they will inevitably be of mixed ion composition, most likely with composition similar to the natural abundances of each isotope and so the linewidth will be smeared and broadened. A linewidth scan is shown in figure 5.28. 


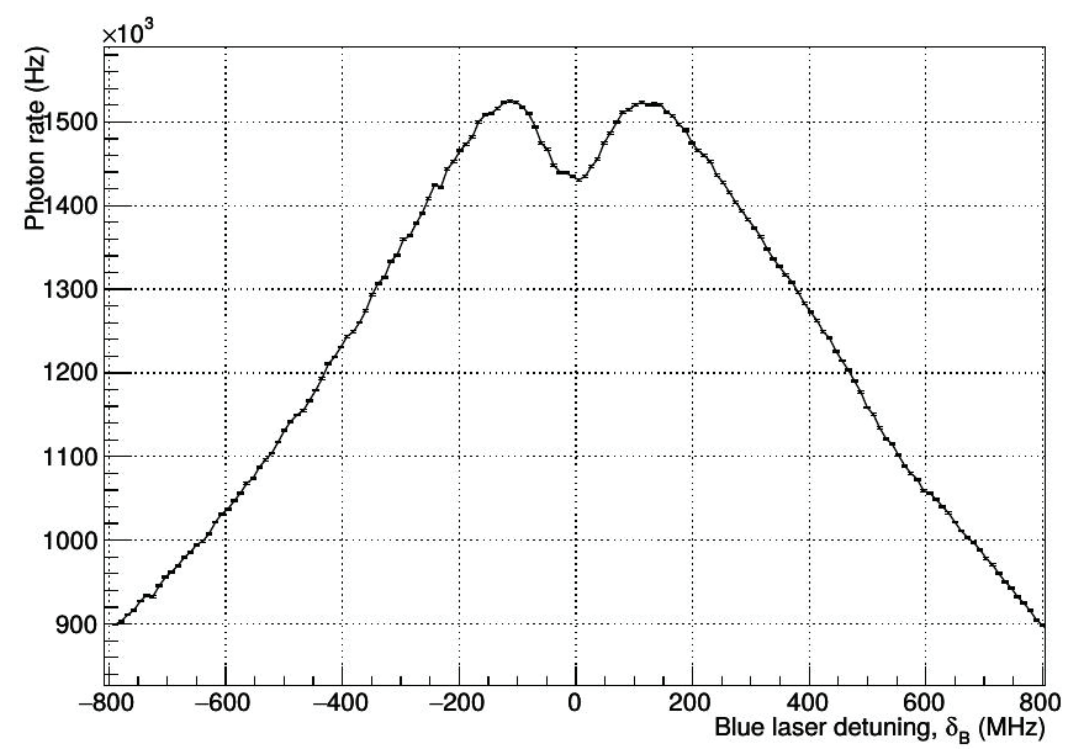

Figure 5.28: An experimentally observed $\mathrm{Ba}^{+}$linewidth with many ions present. This was observed by measuring the fluorescence signal while varying the frequency setpoint of the blue laser. The value of $\delta_{\mathrm{B}}$ here is the detuning from resonance for ${ }^{136} \mathrm{Ba}$.

Another possible method of measuring linewidths would be to monitor the number of ions using the intermodulated technique and then once only a single ion remains, switch to continuous excitation for the linewidth. A couple major obstacles have so far prevented this from producing any results. The primary obstacle is that even if a single ion is known to be present, the ion signal will likely be completely lost in noise from the large background (caused by scattered blue laser light). The other obstacle is the limited lifetime of ions within the trap. Although sometimes an ion may remain trapped upwards of 10 minutes, eventually it will inevitably disappear, either from being ejected due to gas collision or becoming neutralized due to some interaction with other particles. This poses a problem since a linewidth scan take some time to perform (due to the length of each scan and the locking time of the lasers).

A solution currently in progress is the design of a new ion source with the ability to select specific isotopes. By selecting only specific isotopes, the linewidth can be measured with multiple ions present without the smearing effects of mixed isotope composition. To this end, a quadrupole mass spectrometer (QMS) [75] is used to select the specific isotopes. The QMS is currently functional however some difficulty has been encountered in actually producing barium for use with it and work is still ongoing. 


\section{Chapter 6}

\section{Conclusion}

The original intention of this work was to assist in the efforts of both EXO-200 and nEXO in their searches for neutrinoless double-beta decay $(0 \nu \beta \beta)$. To this end improvements were made to the analysis technique used by EXO-200, pioneering a new analysis that will be used for future publications. Furthermore, improvements were made to the barium tagging system in development for use in the upcoming nEXO experiment.

Throughout chapter 4 of this work, a new analysis technique for EXO-200 physics data was detailed. This new technique, which constitutes a large portion of the original work in this thesis, utilized a boosted decision tree (BDT) to combine multiple topological variables into a single value allowing the inclusion of additional information into the EXO-200 analysis at no additional computational cost. It was shown that the BDT was an unbiased discriminator, increasing the separation of signal from background. This discriminator replaced the standoff distance as the second dimension in the 2D fit used previously for EXO-200 analysis resulting in a $13 \%$ improvement to the calculated lower limit on the $0 \nu \beta \beta$ half-lifetime in ${ }^{136} \mathrm{Xe}$ as well as a $12 \%$ improvement in sensitivity when applied to the full Phase-I data set. Due to these improvements this new analysis technique has been approved for use in further analysis including both Phase-I and -II datasets.

Chapter 5 showed the systems used at Carleton for barium tagging. The theory behind ion trapping and couting via two-laser induced fluorescence was presented along with the experimental apparatus used to do so. Two fluorescence schemes were presented, intermodulation and continuous excitation. It was seen that the intermodulation technique was more effective at counting ions whereas the continuous excitation technique did not achieve single ion sensitivity. The system was improved, 
implementing changes to reduce background and increase signal by coating some of the ion trap components with ultra-black anti-reflective coating and focusing the lasers to reduce the beam waist and increase intensity and new ion counting results were presented showing the effects of these improvements. A $50 \%$ improvement in signal/background ratio was seen using the intermodulation technique and some improvement was seen using the continuous excitation technique, approaching the desired single ion sensitivity. Efforts toward measuring the linewidths of different barium isotopes were also presented however this work was not concluded due to the lack of appropriate ion source. This suggests possible future research which could be performed using this same apparatus.

All of the research presented in this work was motivated by the search for the absolute neutrino mass scale as shown in chapter 2. While many experiments are attempting to measure this mass scale, none have yet succeeded and therefore it is important to make as many improvements as possible. The experiments focused upon in this work both show great promise, EXO-200 once held the best upper limit on the Majorana-neutrino mass and the upcoming nEXO, if barium tagging is included, promises great enough sensitivity to entirely exclude the inverted neutrino mass scale (or discover a neutrino mass if it exists there).

While many advancements continue to be made in the search for neutrinoless double-beta decay and the neutrino mass scale, there is always more which can be done. The work presented in this thesis is by no means the end of the line. In the world of EXO-200 analysis there is always work to be done. In the development of the BDT in chapter 4, several variables were pointed out which could not be used due to poor data/MC agreement. While they were not shown to have a very significant impact, their inclusion in a later study (if the Monte Carlo were to be improved) could lead to some further gains. Further improvements to the analysis are likely also possible beside the BDT such as denoising improvements or the development of better background veto systems which would both lead to increased sensitivity.

On the barium tagging side, more work can always be done. While most stages of a full barium tagging system have been built and were shown to perform properly in isolation, they have yet to be combined and tested in one continuous unit. Testing of the full combined system is critical if barium tagging is to be implemented in $\mathrm{nEXO}$ and this is therefore a huge avenue for future study. As well, the extraction of barium from a region of high pressure gas has been demonstrated however a system 
for extraction from a liquid xenon TPC is still under development with a number of methods being studied.

These directions for future study in both EXO-200 and nEXO likely constitute only a fraction of the work that may be pursued (and should be pursued) in followup to this research. In closing, there appears to be a bright future in the field of neutrino physics with many important and exciting research opportunities available. 


\section{List of References}

[1] SNO Collaboration, Measurement of the rate of $\nu_{e}+d \rightarrow p+p+e^{-}$interactions produced by ${ }^{8} B$ solar neutrinos at the Sudbury Neutrino Observatory, Phys. Rev. Lett. 87 (2001)

[2] The Super-Kamiokande Collaboration, Y. Fukuda et al., Evidence for oscillation of atmospheric neutrinos, Phys. Rev. Lett. 81 (1998), pp. 1562-1567

[3] D. Fudenberg, T. Brunner, V. Varentsov, R. Devoe, J. Dilling, G. Gratta and nEXO Collaboration, Barium Tagging for $n E X O$, in APS Division of Nuclear Physics Meeting Abstracts (2015)

[4] C.D. Ellis and W.A. Wooster, The Average Energy of Disintegration of Radium E, Proc. Roy. Soc. A 117 (1927) (776), pp. 109-123

[5] J. Chadwick, Possible Existence of a Neutron, Nature 129 (1932) (312)

[6] E. Fermi, Versuc einer Theorie der $\beta$-Strahlen, Z. fur Physik 88 (1934) (161)

[7] F. Reines, C.L. Cowan, Jr., F.B. Harrison, A.D. McGuire and H.W. Kruse, Detection of the Free Antineutrino, Phys. Rev. 117 (1960) (159)

[8] M. Goldhaber, L. Grodzins and A.W. Sunyar, Helicity of Neutrinos, Phys. Rev. Lett. 9 (1958), pp. 36-44

[9] K. Kodama et al., Observation of Tau Neutrino Interactions, Phys. Rev. Lett. B 504 (2001), pp. 218-224

[10] B. Pontecorvo, Neutrino Experiments and the Problem of Conservation of Leptonic Charge, Sov. Phys. JETP 26 (1968) (984)

[11] K. Lande, E.T. Booth, J. Impeduglia, L.M. Lederman and W. Chinowksy, Observation of Long-Lived Neutral V Particles, Phys. Rev. 103 (1956) (1901)

[12] F. Capozzi, G.L. Fogli, E. Lisi, A. Marrone, D. Montanino and A. Palazzo, Status of three-neutrino oscillation parameters, circa 2013, Phys. Rev. D 89 (2014)

[13] M. Green, On ion probes and traps: Barium tagging for the EXO double beta decay detector, Ph.D. thesis, Stanford University (2010)

[14] Felix Boehm and Peter Vogel, Physics of Massive Neutrinos, 2nd ed. (Cambridge University Press, 1992) 
[15] KamLAND-Zen Collaboration, Search for Majorana Neutrinos near the Inverted Mass Hierarchy Region with KamLAND-Zen, Phys. Rev. Lett. 117 (2016)

[16] J.B. Albert et al., Search for Majorana neutrinos with the first two years of EXO-200 data, Nature 510 (2014), pp. 229-234

[17] M. Agostini et al., Background free search for neutrinoless double beta decay with GERDA Phase II, Nature 544 (2017) (7648), pp. 5-132

[18] K. Alfonso et al., Search fpr Neutrinoless Double-Beta Decay of ${ }^{130}$ Te with CUORE-0, Phys. Rev. Lett. 115 (2015)

[19] R. Killick, Observation of singly charged barium ions in a buffer gas, Master's thesis, Carleton University (2015)

[20] J. Kotila and F. Iachello, Phase-space factors for double- $\beta$ decay, Phys. Rev. C $85(2012)$

[21] J. Barea, J. Kotila and F. Iachello, $0 \nu \beta \beta$ and $2 \nu \beta \beta$ nuclear matrix elements in the interaction boson model with isospin restoration, Phys. Rev. C 91 (2015)

[22] Fedor S̆imkovic, Vadim Rodin, Amand Faessler and Petr Vogel, $0 \nu \beta \beta$ and $2 \nu \beta \beta$ nuclear matrix elements, quasiparticle random-phase approximation, and isospin symmetry restoration, Phys. Rev. C 87 (2013)

[23] J.B. Albert et al., An improved measurement of the $2 \nu \beta \beta$ half-life of ${ }^{136}$ Xe with EXO-200, Phys. Rev. C 89 (2014)

[24] 3M HFE-7000, http://www.3m.com

[25] M. Auger et al., The EXO-200 detector, part I: detector design and construction, Journal of Instrumentation 7 (2012)

[26] J.B. Albert et al., Measurement of the Drift Velocity and Transverse Diffusion of Electrons in Liquid Xenon with the EXO-200 Detector, Phys. Rev. C 95 (2017)

[27] J.B. Albert et al., Search for $2 \nu \beta \beta$ decay of ${ }^{136} X$ e to the $0_{1}^{+}$excited state of ${ }^{136} B a$ with EXO-200, Phys. Rev. C 93 (2016)

[28] Byron P. Roe, Hai-Jun Yang, Ji Zhu, Yong Liu, Ion Stancu and Gordon McGregor, Boosted Decision Trees as an Alternative to Artificial Neural Networks for Particle Identification, Nucl. Instrum. Meth. 543 (2005)

[29] ATLAS Collaboration, Measurement of the Higgs boson mass from the $H \rightarrow \gamma \gamma$ and $H \rightarrow Z Z^{*} \rightarrow 4 l$ channels with the ATLAS detector at the LHC, Phys. Rev. D (2014)

[30] ATLAS Collaboration, Prospective results for vector-boson fusion-mediated Higgs-boson searches in the four lepton final state at the High Luminosity Large Hadron Collider, ATLAS-PHYS-PUB-2016-008 (2016) 
[31] David Di Valentino, Search for the Standard Model Higgs boson in the vector boson fusion-mediated diphoton decay channel using multivariate analysis techniques, Master's thesis, Carleton University (2013)

[32] A. Hoecker, P. Speckmayer, J. Stelzer, J. Therhaag, E. von Toerne and H. Voss, TMVA 4 Toolkit for Multivariate Data Analysis with ROOT User's Guide

[33] F.J. Massey, The Kolmogorov-Smirnov Test for Goodness of Fit, Journal of the American Statistical Association 46 (1951) (253), pp. 68-78

[34] Geant4 Collaboration, Geant4 User's Guide for Application Developers

[35] F. James and M. Winkler, Minuit 2, CERN, Geneva

[36] W. Verkerke and D. Kirkby, RooFit Users Manual v2.91

[37] J.B. Albert et al., Search for Neutrinoless Double-Beta Decay in ${ }^{136}$ Xe with the Upgraded EXO-200 Detector (2017), unpublished draft

[38] S. S. Wilks, The large-sample distribution of the likelihood ratio for testing composite hypotheses, Ann. Math. Statist. 9 (1938), pp. 60-62

[39] R. Killick, nEXO Discovery Potential, unpublished meeting slides for the nEXO MC group

[40] T. Brunner et al., An RF-only ion-funnerl for extraction from high-pressure gases, Int. J. Mass Spect. 379 (2015), pp. 110-120

[41] J. Wodin, Single ion trapping in a buffer gas and liquid Xe energy resolution studies for the EXO experiment, Ph.D. thesis, Stanford University (2007)

[42] E. Rollin, Barium ion extraction and identification from laser induced fluorescence in gas for the Enriched Xenon Observatory, Ph.D. thesis, Carleton University (2011)

[43] W. Fairbank Jr., Ba Tagging summary: new results and plans (2017), unpublished meeting slides for nEXO barium tagging group

[44] Edwards Vacuum, Instruction Manual nEXT Turbomolecular Pumps nEXT240, nEXT300 and nEXT400 (2009)

[45] Pfeiffer Vacuum, PKR 261 Compact FullRange ${ }^{T M}$ Gauge, All-metal (2013)

[46] BK PRECISION, Model 4005DDS 5 MHz DDS Function Generator INSTRUCTION MANUAL

[47] Hewlett-Packard, 6227B Operating and Service Manual (1970)

[48] Extrel CMS, L.P., 5221 MS Controller Hardware Reference Manual (2004)

[49] Pfeiffer Vacuum, UDV 146 All-Metal Control Valve, thermomechanically and manually actuated Operating Instructions (2010) 
[50] SAES Pure Gas, Inc., A member of the SAES Getters Group, Product Manual MICROTORR Point-of-Use Ambient Temperature Purifiers

[51] Y. Wu and X. Yang, Strong-Coupling Theory of Periodically Driven Two-Level Systems, Phys. Rev. Lett. 98 (2007)

[52] G. Janik, W. Nagourney and H. Dehmelt, Doppler-free optical spectroscopy of the $\mathrm{Ba}^{+}$mono-ion oscillator, Journal of the Optical Society of America B 2 (1985) (8), pp. 1251-1257

[53] D. De Munshi, T. Dutta, R. Rebhi and M. Mukherjee, Precision measurement of branching fractions of $138 \mathrm{Ba}+$ : Testing many-body theories below the 1\% level, Phys. Rev. A 91 (2015)

[54] K. Wendt, S. A. Ahmad, F. Buchinger, A.C. Mueller, R. Neugart and E.-W. Otten, Relativistic J-dependence of the isotope shift in the 6s-6p doublet of $B a$ II, Z. Phys. A - Atoms and Nuclei 318 (1984), pp. 125-129

[55] P. Villemoes, A. Arnesen, F. Heijkenskjold and A. Wannstrom, Isotope shifts and hyperfine structure of 134-138Ba II by fast ion beam-laser spectroscopy, J. Phys. B: At. Mol. Opt. Phys. 26 (1993), pp. 4289-4299

[56] TOPTICA Photonics AG, TA pro Tapered Amplifier System Manual (2011)

[57] TOPTICA Photonics AG, TA/DL-SHG pro Frequency Doubled High Power Laser System Manual (2009)

[58] TOPTICA Photonics AG, DL pro Grating Stabilized Diode Laser Head Manual (2011)

[59] HighFinesse, Wavelength Meter Angstrom WS Ultimate 10 Autocalibration Singlemode Operation User Manual

[60] SIOS Meßtechnik GmbH, Instruction Manual Model SL 03 Frequency-Stabilized He-Ne-Lasers (2001)

[61] R. Drever, J.L. Hall, F.V. Kowalski, J. Hough, G.M. Ford, A.J. Munley and H. Ward, Laser phase and frequency stabilization using an optical resonator, Applied Physics B 31 (1983) (2)

[62] Newport Corporation, LBP Series Laser Beam Profiler for Windows User's Manual

[63] NEOS Technologies, Inc., OPERATING MANUAL AO MODULATOR MODEL NUMBER: 35085-0.5 OR 35085-0.5-350

[64] NEOS Technologies, Inc., OPERATING MANUAL ACOUSTO OPTIC MODULATOR MODEL NUMBER: 23080-1 23080-X-LTD X=1,2, or $3 \mathrm{~mm}$

[65] Newport Corporation, 1830-C Optical Power Meter Instruction Manual (1994)

[66] H. Photonics, Metal Package PMT with Cooler, Photosensor Modules H7422 Series, Product Datasheet 
[67] Keithley Instruments Inc., Model 6517B Electrometer User's Manual (2008)

[68] Tektronix Inc., User Manual Tektronix TDS3000 Series Digital Phosphor Oscilloscopes

[69] Advanced Research Instruments Corporation, USER MANUAL for the F-100T PREAMPLIFIER/DISCRIMINATOR V9 (2005)

[70] ORTEC, Easy-MCSTM with the MCS-32 (A73-B32) Software for Microsoft ${ }^{\circledR}$ 7 and Windows XP ${ }^{\circledR}$ SP3, Hardware and Software User's Manual, Software Version 2.2

[71] Spectra-Physics, A Division of Newport Corporation, Explorer Actively QSwitched, High-Repetition Laser Systems User's Manual (2008)

[72] A. Ltd., Acktar Black ${ }^{T M}$ World's Blackest Coatings By Industrial Vacuum Deposition Technology, Technical Datasheet

[73] F. Schmidt-Kaler, H. Häffner, S. Gulde, M. Riebe, G.P.T. Lancaster, T. Deuschle, C. Becher, W. Hänsel, J. Eschner, C.F. Roos and R. Blatt, How to realize a universal quantum gate with trapped ions, Applied Physics B 77 (2003) (8)

[74] Y. Lan, Linear Paul trap progress update: Ion ejection and ion temperature (2017), unpublished meeting slides for Carleton barium tagging group

[75] Extrel CMS, L.P., Flange Mounted Mass Spectrometer Hardware Installation Guide (2004) 